\title{
Search for dark matter candidates and large extra dimensions in events with a jet and missing transverse momentum with the ATLAS detector
}

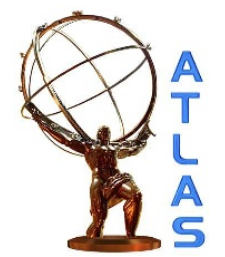

\section{The ATLAS collaboration}

\section{E-mail: atlas.publications@cern.ch}

ABstRACT: A search for new phenomena in events with a high-energy jet and large missing transverse momentum is performed using data from proton-proton collisions at $\sqrt{s}=7 \mathrm{TeV}$ with the ATLAS experiment at the Large Hadron Collider. Four kinematic regions are explored using a dataset corresponding to an integrated luminosity of $4.7 \mathrm{fb}^{-1}$. No excess of events beyond expectations from Standard Model processes is observed, and limits are set on large extra dimensions and the pair production of dark matter particles.

KEYWORDS: Hadron-Hadron Scattering

ARXIV EPRINT: 1210.4491 


\section{Contents}

1 Introduction 1

2 Data and simulated samples $\quad 4$

3 Analysis strategy and physics object reconstruction 5

$\begin{array}{llr}4 & \text { Event selection } & 8\end{array}$

5 Background estimation $\quad 9$

$\begin{array}{ll}5.1 \text { Backgrounds from } Z / W+\text { jets } & 10\end{array}$

$\begin{array}{lll}5.2 & \text { Multijet backgrounds } & 14\end{array}$

$\begin{array}{lll}5.3 & \text { Non-collision backgrounds } & 14\end{array}$

$\begin{array}{ll}5.4 & \text { Systematic uncertainties on background estimates } \\ 5.5 & 14\end{array}$

$\begin{array}{ll}\text { 5.5 Background summary and additional checks } & 15\end{array}$

$\begin{array}{llr}6 & \text { Results and interpretation } & 17\end{array}$

$\begin{array}{lll}6.1 & \text { Large extra dimensions } & 18\end{array}$

$\begin{array}{ll}6.2 & \text { WIMP pair production } \\ & 22\end{array}$

$\begin{array}{lll}7 & \text { Summary } & 27\end{array}$

$\begin{array}{ll}\text { The ATLAS collaboration } & 35\end{array}$

\section{Introduction}

Event topologies with a single jet with large transverse energy and large missing transverse momentum, referred to as monojets in the following, are important final states for searches for new phenomena beyond the Standard Model (SM) at a hadron collider. The primary SM process that results in a true monojet final state is $Z$-boson production in association with a jet, where the $Z$ boson decays to two neutrinos. A further important reducible contribution to this final state consists of events that include a $W$ boson and a jet, where the charged lepton from the $W$-boson decay is not reconstructed.

Phenomenological scenarios beyond the Standard Model (BSM) that result in a monojet final state include supersymmetry [1-11] and large extra dimensions (LED) [12]. A model-independent treatment of the production of dark matter (DM) particles at the Large Hadron Collider (LHC) has been proposed recently, where DM particles are pair-produced in association with a jet [13-15]. In the following, a search for an excess of monojet events over SM expectations is performed. The results are interpreted in a framework of LED and DM particle pair production. They are based on a dataset of $4.7 \mathrm{fb}^{-1}$ of proton-proton 
$(p p)$ collisions at $\sqrt{s}=7 \mathrm{TeV}$ recorded with ATLAS at the LHC and supersede those presented in the 2010 ATLAS monojet analysis that used $35 \mathrm{pb}^{-1}$ of data [16]. Other monojet searches were performed in Run I and Run II at the Tevatron [17-19] and also by CMS with the 2010 [20] and 2011 [21] LHC datasets. None of these found evidence of new phenomena beyond the Standard Model.

Models of large extra spatial dimensions have been proposed to remove the hierarchy problem [22-25] by addressing the weakness of gravity relative to all other forces. One popular model of LED that is often used to interpret the results of monojet searches at particle colliders is that of Arkani-Hamed, Dimopoulos, Dvali (ADD) [12]. In this model, gravity propagates in the $(4+n)$-dimensional bulk of space-time, while the SM fields are confined to four dimensions. The large observed difference between the characteristic mass scale of gravity (the Planck mass) and the electroweak scale (as characterised by the $W$-boson mass) is the result of the four-dimensional interpretation of the Planck scale, $M_{\mathrm{Pl}}=1.2 \times 10^{19} \mathrm{GeV}$, which is related to the fundamental $(4+n)$-dimensional Planck scale $\left(M_{\mathrm{D}}\right)$ by $M_{P l}{ }^{2}=8 \pi M_{\mathrm{D}}{ }^{2+n} R^{n}$, where $n$ and $R$ are the number and size of the extra dimensions, respectively. An appropriate choice of $R$ for a given $n$ results in a value of $M_{\mathrm{D}}$ close to the electroweak scale. The extra spatial dimensions are compactified, resulting in a Kaluza-Klein tower of massive graviton modes. At hadron colliders, these graviton modes can be produced in association with a jet. The production processes include $q g \rightarrow q G, g g \rightarrow g G$, and $q \bar{q} \rightarrow g G$, where $G$ stands for the tower of gravitons, $q$ for a quark, and $g$ for a gluon. As gravitons do not interact with the detector, these processes give rise to a monojet signature [26].

Particle dark matter is a well-established paradigm to explain a range of astrophysical measurements (see for example ref. [27] for a recent review). Since none of the known SM particles are adequate DM candidates, the existence of a new particle is hypothesised, with properties suitable to explain the astrophysical measurements. One class of particle candidates of interest for searches at the LHC consists of weakly interacting massive particles (WIMPs) [28]. These are expected to couple to SM particles through a generic weak interaction, which could be the known weak interaction of the SM or a new type of interaction. Such a new particle is a cold dark matter candidate, which can be produced at the LHC. It results in the correct relic density values for non-relativistic matter in the early universe [29], as measured by the WMAP satellite [30], if its mass lies in the range between a few $\mathrm{GeV}$ and a $\mathrm{TeV}$ and if it has electroweak-scale interaction cross sections. The fact that a new particle with such properties can be a thermal relic of the early universe in accordance with the WMAP measurements is often referred to as the WIMP miracle. Many new particle physics models designed to solve the hierarchy problem also predict WIMPs.

Because WIMPs do not interact with the detector material, their production leads to signatures with missing transverse momentum $\left(\mathbf{p}_{\mathrm{T}}^{\mathrm{miss}}\right),{ }^{1}$ the magnitude of which is called $E_{\mathrm{T}}^{\mathrm{miss}}$. Searches involving $E_{\mathrm{T}}^{\mathrm{miss}}$ at the LHC are therefore canonical WIMP searches, although the LHC experiments cannot establish whether a WIMP candidate is stable on cosmological time scales and hence a DM candidate. In some supersymmetric models, WIMPs are expected to be dominantly produced in cascade decays of heavier unstable

\footnotetext{
${ }^{1}$ Letters in bold font are used for vector quantities.
} 


\begin{tabular}{|c|ccc|}
\hline Name & Initial state & Type & Operator \\
\hline D1 & $q q$ & scalar & $\frac{m_{q}}{M_{\star}^{3}} \bar{\chi} \chi \bar{q} q$ \\
D5 & $q q$ & vector & $\frac{1}{M_{\star}^{2}} \bar{\chi} \gamma^{\mu} \chi \bar{q} \gamma_{\mu} q$ \\
D8 & $q q$ & axial-vector & $\frac{1}{M_{\star}^{2}} \bar{\chi} \gamma^{\mu} \gamma^{5} \chi \bar{q} \gamma_{\mu} \gamma^{5} q$ \\
D9 & $q q$ & tensor & $\frac{1}{M_{\star}^{2}} \bar{\chi} \sigma^{\mu \nu} \chi \bar{q} \sigma_{\mu \nu} q$ \\
D11 & $g g$ & scalar & $\frac{1}{4 M_{\star}^{3}} \bar{\chi} \chi \alpha_{s}\left(G_{\mu \nu}^{a}\right)^{2}$ \\
\hline
\end{tabular}

Table 1. Effective interactions coupling Dirac fermion WIMPs to Standard Model quarks or gluons, following the formalism of ref. [32]. The tensor operator D9 describes a magnetic-moment coupling. The factor of the strong coupling constant $\alpha_{s}$ in the definition of D11 accounts for this operator being induced at one-loop level. $G_{\mu \nu}$ is the colour field-strength tensor.

supersymmetric particles along with high transverse momentum $\left(p_{\mathrm{T}}=\left|\mathbf{p}_{\mathrm{T}}\right|\right) \mathrm{SM}$ particles. In a more model-independent approach, WIMP pair production at colliders is proposed to yield detectable $E_{\mathrm{T}}^{\mathrm{miss}}$ if the WIMP pair is tagged by a jet or photon from initial- or finalstate radiation (ISR/FSR) [13,31]. Even though this approach does not rely on a specific BSM scenario, it does have assumptions: WIMPs are pair-produced at the LHC and all new particles mediating the interaction between WIMPs and the SM are too heavy to be produced directly; they can thus be integrated out in an effective field theory approach. The resulting interaction is hence a contact interaction between the dark sector and the SM. It is worth noting that the DM particles are not explicitly assumed to interact via the weak force. They may also couple to the SM via a new force. Throughout this work, the terms WIMP and DM particle (candidate) are synonymous.

It is assumed here that the DM particle is a Dirac fermion $\chi$, where the only difference for Majorana fermions would be that certain interaction types are not allowed and that the cross section for each operator is larger by a factor of four. Five interactions are considered (table 1), namely D1, D5, D8, D9, D11, following the naming scheme of ref. [32]. D1, D5, D8, and D9 describe different bilinear quark couplings to WIMPs, $q q \rightarrow \chi \chi$, and D11 describes the process $g g \rightarrow \chi \chi$. The 14 operators for Dirac fermions in ref. [32] fall into four categories with characteristic $E_{\mathrm{T}}^{\text {miss }}$ spectral shapes. D1, D5, D9, and D11 are a representative set of operators for these four categories, while D8 falls into the same category as D5 but is listed explicitly in table 1 because it is often used to convert LHC limits into limits on DM pair production. In the operator definitions in table $1, M_{*}$ is the suppression scale of the heavy mediator particles that are integrated out. The use of a contact interaction to produce WIMP pairs via heavy mediators is considered conservative because it rarely overestimates cross sections when applied to a specific BSM scenario. Cases where this approach is indeed optimistic are studied in refs. [15, 33]. The effective theory provides a useful framework for comparing LHC results to direct or indirect dark matter searches. Within this framework, interactions of SM and DM particles are described by only two parameters, the suppression scale $M_{*}$ and the DM particle mass $m_{\chi}$. 


\begin{tabular}{|l|llll|}
\hline Process & Generator & Parton shower & Underlying event & PDF \\
\hline$Z / W+$ jets & ALPGEN & HERWIG & JIMMY & CTEQ6L1 \\
$t \bar{t}$, single $t$ & MC@NLO & HERWIG & JIMMY & CTEQ6.6 \\
Di-boson & SHERPA & SHERPA & SHERPA & CTEQ6L1 \\
ADD & PYTHIA & PYTHIA & PYTHIA & MSTW2008 LO** /CTEQ6.6 \\
WIMPs & MADGRAPH5 & PYTHIA & PYTHIA & CTEQ6L1 \\
\hline
\end{tabular}

Table 2. Overview of the main simulated samples.

\section{Data and simulated samples}

The ATLAS detector [34, 35] at the LHC covers the pseudorapidity ${ }^{2}$ range of $|\eta|<4.9$ and all of $\phi$. It consists of an inner tracking detector surrounded by a thin superconducting solenoid, electromagnetic and hadronic calorimeters, and an external muon spectrometer incorporating large superconducting toroidal magnets. A three-level trigger system is used to select interesting events for recording and subsequent offline analysis. Only data for which all subsystems described above were operational are used. Applying these requirements to $p p$ collision data, taken at a centre-of-mass energy of $\sqrt{s}=7 \mathrm{TeV}$ with stable beam conditions during the 2011 LHC run, results in a data sample with a time-integrated luminosity of $4.7 \mathrm{fb}^{-1}$, determined with an uncertainty of $3.9 \%[36,37]$.

Monte Carlo (MC) simulations are used both as part of the background estimation and to model signal processes. Processes that dominate the background are $Z$ - or $W$ boson production in association with jets, which are simulated with ALPGEN [38] using the parton distribution function (PDF) set CTEQ6L1 [39]. The $W \rightarrow \ell \nu$ plus jets and $Z \rightarrow \nu \bar{\nu}$ plus jets samples are simulated with up to six additional partons at leading order, while the process $Z / \gamma^{*} \rightarrow \ell^{+} \ell^{-}$plus jets is simulated with up to five additional partons at leading order. Additional jets are generated via parton showering, which, together with fragmentation and hadronisation, is performed by HERWIG [40, 41]. The MLM [42] prescription is used for matching the matrix-element calculations to the parton shower evolution. JIMMY [43] is used to simulate the underlying event. Additional $Z / W$ plus jets samples generated with SHERPA [44] are used to estimate the uncertainties related to the event generator. Single top quark and pair production are simulated with MC@NLO [45], fixing the top-quark mass to $172.5 \mathrm{GeV}$, and using the next-to-leading-order (NLO) PDF set CTEQ6. 6 [46]. Parton showering and hadronisation are performed with HERWIG, and JIMMY is again used for the underlying event. Di-boson $(W W, W Z, Z Z)$ samples are generated with SHERPA. Backgrounds from QCD multijet production are estimated from data (see section 5.2 ). PYTHIA [47] simulations of this process, normalised to data, are used in figures for illustrative purposes only.

\footnotetext{
${ }^{2}$ ATLAS uses a right-handed coordinate system with its origin at the nominal interaction point (IP) in the centre of the detector and the $z$-axis along the beam pipe. The $x$-axis points from the IP to the centre of the LHC ring, and the $y$-axis points upward. Polar coordinates $(r, \phi)$ are used in the transverse $(x, y)$-plane, $\phi$ being the azimuthal angle around the beam pipe. The pseudorapidity is defined in terms of the polar angle $\theta$ as $\eta=-\ln \tan (\theta / 2)$.
} 
For graviton production in the ADD model, a low-energy effective field theory [26] with energy scale $M_{\mathrm{D}}$ is used to calculate the signal cross section considering the contribution of different graviton mass modes. Signal samples corresponding to a number of extra dimensions varying between two and six are considered, with the renormalisation and factorisation scales set per event to $\sqrt{\frac{1}{2} M_{\mathrm{G}}^{2}+p_{\mathrm{T}}^{2}}$, where $M_{\mathrm{G}}$ is the mass of the graviton mode produced in the event and $p_{\mathrm{T}}$ denotes the transverse momentum of the recoiling parton. The samples are produced with an ADD implementation as a user model of PYTHIA, which is also used for parton showering and hadronisation. MSTW2008 LO** [48] PDF sets are used for the event simulation. The event yields for CTEQ6.6 PDFs are obtained by reweighting these samples, and are used to estimate cross sections, as well as PDF systematic uncertainties. ADD cross sections are calculated at both leading order (LO) and NLO. The NLO calculations take into account QCD corrections to graviton production and have been produced for the kinematic regions explored here following ref. [49].

The effective field theory of WIMP pair production is implemented in MADGRAPH5 [50] (version 1.3.33), taken from ref. [32]. WIMP pair production plus one and two additional partons from ISR/FSR is simulated requiring at least one parton with a minimum transverse momentum of $80 \mathrm{GeV}$. Only initial states of gluons and the four lightest quarks are considered, assuming equal coupling strengths for all quark flavours to the WIMPs. The mass of charm quarks is most relevant for the cross sections of the operator D1 (see table 1) and it is set to $1.42 \mathrm{GeV}$. The generated events are interfaced to PYTHIA for parton showering and hadronisation. The MLM prescription is used for matching the matrix-element calculations of MADGRAPH5 to the parton shower evolution of PYTHIA. The CTEQ6L1 PDF set is used for the event simulation. The MADGRAPH5 default choice for the renormalisation and factorisation scales is used. The scales are set to the sum of $\sqrt{m^{2}+p_{\mathrm{T}}^{2}}$ for all produced particles, where $m$ is the mass of particles. Events with WIMP masses between 10 and $1300 \mathrm{GeV}$ are simulated for four different effective operators (D1, D5, D9, D11). In all cases, WIMPs are taken to be Dirac fermions, and the pair-production cross section is calculated at LO.

The background MC samples use a detector simulation [51] based on GEANT4 [52] and are reconstructed with the same algorithms as the data. The signal MC samples employ a mix of the detailed GEANT4 detector simulation and a simulation relying on parametrisations of calorimetric signals to shorten the CPU time required (ATLFAST-II [51]). Individual signal MC samples have been validated against the more detailed detector simulation relying fully on GEANT4. Effects of event pile-up - multiple $p p$ interactions occurring in the same or neighbouring crossing of two proton bunches, called pile-up from now on - are included in the simulation. MC events are re-weighted to reproduce the distribution of the number of collisions per bunch crossing observed in the data.

\section{Analysis strategy and physics object reconstruction}

A search for a BSM excess is performed in monojet final states. Leptons are vetoed to suppress background contributions from $Z / W$ plus jets. A second jet is allowed as long as 
it is not aligned with $E_{\mathrm{T}}^{\text {miss }}$, which would be the case in multijet background events with a mis-measured jet. Events with more than two jets are vetoed.

These selection requirements define the basic signal region (SR), defined in detail in table 3 below. The data sample consisting of events passing the SR selections is sub-divided into overlapping kinematic regions by applying selection criteria on $E_{\mathrm{T}}^{\mathrm{miss}}$ and $p_{\mathrm{T}}^{\mathrm{jet} 1}$, the transverse momentum of the most energetic jet (leading jet) in the event. Events in these overlapping individual signal regions are then used to search for a BSM excess above the predicted SM backgrounds.

The main SM background contributions to the SR data samples are from $Z / W+$ jets production and they are estimated from data by selecting events based on a set of selection requirements - orthogonal to those of the signal regions - that define a control region (CR). These CR requirements are based on selecting events with leptons (either exactly one or two leptons). Different physics processes are used to estimate background contributions to a SR: $W \rightarrow e \nu+$ jets, $W \rightarrow \mu \nu+$ jets, $Z \rightarrow e^{+} e^{-}+$jets, and $Z \rightarrow \mu^{+} \mu^{-}+$jets. To obtain data samples enriched by these processes, a set of selection criteria defines corresponding CR's. Each set of CR requirements is further sub-divided into the same kinematic categories as the signal regions.

This analysis is based on reconstructed jets, electrons, muons, and $E_{\mathrm{T}}^{\text {miss }}$. The definitions of electron and muon candidates and of $E_{\mathrm{T}}^{\text {miss }}$ are different in the SR and CR requirements. All electron candidates are required to have $p_{\mathrm{T}}>20 \mathrm{GeV}$ and $|\eta|<2.47$, in order to be within the acceptance of the tracking system. For the signal-region requirements, which comprise an electron veto, relatively loose criteria are used to define an electron candidate (SR-electron), because a looser electron definition leads to a more stringent veto. SR-electrons are required to pass the medium electron shower shape and track selection criteria described in ref. [56]. No spatial isolation is required. For the control-region selection requirements, used for background estimates from events with measured electrons, more stringent electron selection criteria (defining the CR-electron) are used in case exactly one electron is selected. This is to better suppress jet contamination in these control regions. A CR-electron is required to pass the tight [56] electron shower shape and track selection criteria in $W \rightarrow e \nu$ control regions. In addition, the following isolation criterion is imposed for CR-electrons to suppress events where a jet is mis-identified as an electron: the scalar sum of the transverse momentum of tracks with $\Delta R \equiv \sqrt{(\Delta \phi)^{2}+(\Delta \eta)^{2}}<0.2$ around the electron candidate, excluding the electron itself, has to be less than $10 \%$ of the electron's transverse energy $\left(E_{\mathrm{T}}\right)$. In control regions where exactly two electrons are required, the looser SR-electron definition without isolation requirements is used.

A muon candidate used in the definition of the signal regions (SR-muon) is reconstructed either by associating a stand-alone muon spectrometer track with an inner detector track, or from an inner detector track that is confirmed by a directional segment in the muon spectrometer [57]. SR-muons, which are used as veto in signal-region selections, are required to have $p_{\mathrm{T}}>7 \mathrm{GeV}$ and $|\eta|<2.5$. They are also required to be isolated: the scalar $p_{\mathrm{T}}$ sum of tracks within $\Delta R=0.2$ around the muon track, excluding the muon itself, must be less than $1.8 \mathrm{GeV}$. As for electrons, the muon selection criteria in control-regions definitions are more stringent. A CR-muon candidate must have a stand-alone muon spec- 
trometer track associated with an inner detector track. Those SR-muons that have only an inner detector track tagged by a segment in the muon spectrometer do not satisfy the CR selection criteria. Furthermore, CR-muons satisfy $p_{\mathrm{T}}>20 \mathrm{GeV}$ and $|\eta|<2.4$, and have an impact parameter along $z$ with respect to the reconstructed primary vertex of $\left|z_{0}\right|<10 \mathrm{~mm}$ to reject cosmic-ray muons. The CR-muons are also required to be isolated: the scalar $p_{\mathrm{T}}$ sum of tracks within $\Delta R=0.2$ around the muon track, excluding the muon itself, must be less than $10 \%$ of the muon $p_{\mathrm{T}}$.

In the signal regions, the measurement of $E_{\mathrm{T}}^{\mathrm{miss}}$ is performed using all clusters of energy deposits in the calorimeter up to $|\eta|$ of 4.5. The calibration of these clusters takes into account the different response of the calorimeters to hadrons compared to electrons or photons, as well as dead material and out-of-cluster energy losses [58, 59]. In the control regions, two additional definitions of $E_{\mathrm{T}}^{\text {miss }}$ are used to account for the different treatment in the signal and control regions of electrons and muons. This is because the calorimetric definition of the nominal $E_{\mathrm{T}}^{\text {miss }}$ takes into account energy deposits of electrons whereas it does not account for transverse momentum carried away by muons. The two additional definitions of $E_{\mathrm{T}}^{\text {miss }}$ either exclude the electron contributions to the missing transverse momentum in events with electrons or include the muon contributions to the missing transverse momentum in events with muons:

- $E_{\mathrm{T}}^{\text {miss, },}$ : for control regions that involve electrons $\left(W \rightarrow e \nu+\right.$ jets, $Z \rightarrow e^{+} e^{-}+$jets, explained in more detail below), $E_{\mathrm{T}}^{\text {miss, } \mathscr{f}}$ is obtained by adding the electron clusters to the missing transverse momentum vector thereby removing the electron contribution to the calculation of $E_{\mathrm{T}}^{\text {miss }}: E_{\mathrm{T}}^{\text {miss, } \mathscr{\phi}}=\left|\mathbf{p}_{\mathrm{T}}^{\text {miss }}+\mathbf{p}_{\mathrm{T}}^{\text {electrons }}\right|$. This yields missing transverse momentum which, as in invisible $Z$ decays, does not take into account the decay products of the $Z$ boson.

- $E_{\mathrm{T}}^{\mathrm{miss}, \mu}$ : the second alternative version of $E_{\mathrm{T}}^{\text {miss }}$ takes into account the muon contribution to $E_{\mathrm{T}}^{\mathrm{miss}}$ and it is used in the exclusive $W \rightarrow \mu \nu+$ jets control regions. It is defined as the negative sum of the calorimeter-based $\mathbf{p}_{\mathrm{T}}^{\text {miss }}$ and the transverse momentum of muons, which do not deposit much energy in the calorimeters: $E_{\mathrm{T}}^{\mathrm{miss}, \mu}=\left|\mathbf{p}_{\mathrm{T}}^{\mathrm{miss}}-\mathbf{p}_{\mathrm{T}}^{\text {muons }}\right|$.

With these three versions of missing transverse momentum, the kinematics of invisible $Z \rightarrow \nu \bar{\nu}$ decays can be mimicked in $Z$ or $W$ events with measured muons $\left(E_{\mathrm{T}}^{\mathrm{miss}}\right)$ or electrons $\left(E_{\mathrm{T}}^{\text {miss, },}\right)$. On the other hand, for the selection of such control samples enriched with $Z$ or $W$ events, the missing transverse momentum taking into account all visible decay products of $Z$ or $W$ bosons can be used in events with measured muons $\left(E_{\mathrm{T}}^{\text {miss, } \mu}\right)$ or electrons $\left(E_{\mathrm{T}}^{\mathrm{miss}}\right)$.

Jet candidates are reconstructed using the anti- $k_{t}$ clustering algorithm [53] with a radius parameter of 0.4 . The inputs to this algorithm are clusters of energy deposits in calorimeter cells seeded by those with energies significantly above the measured noise [54]. Jet momenta are calculated by performing a four-vector sum over these cell energy clusters, treating each cluster as an $(E, \mathbf{p})$ four-vector with zero mass. The direction of $\mathbf{p}$ is given by the line joining the nominal interaction point with the calorimeter cluster. The result- 
ing jet energies are corrected to the hadronic scale using $p_{\mathrm{T}}$ and $\eta$ dependent calibration factors based on MC simulations and validated by extensive test beam and collision data studies [55].

\section{Event selection}

All data passing detector quality requirements are considered for the analysis. Events must be accepted by an inclusive $E_{\mathrm{T}}^{\text {miss }}$ trigger $[60,61]$ that is found to be $98 \%$ efficient for events with $E_{\mathrm{T}}^{\text {miss }}$ above $120 \mathrm{GeV}$, and more than $99 \%$ for $E_{\mathrm{T}}^{\text {miss }}$ above $150 \mathrm{GeV}$. At $120 \mathrm{GeV}$, a small residual dependence on pile-up of the $E_{\mathrm{T}}^{\text {miss }}$ trigger efficiency is found. Over the full 2011 dataset, where the pile-up varied from an average of 3 interactions per bunch crossing at the beginning of the year to 17 at the end of the year, an efficiency variation of $1.5 \%$ is observed and a correction is applied to account for this variation. For $E_{\mathrm{T}}^{\mathrm{miss}}$ above $220 \mathrm{GeV}$, there is no measurable efficiency variation. Events are further required to satisfy a set of pre-selection and kinematic criteria that are aimed at selecting monojet events from good-quality $p p$ collisions, as well as reducing electroweak, multijet, non-collision, and detector-induced backgrounds. These criteria require the event to have a monojet topology characterised by one unbalanced high- $p_{\mathrm{T}}$ jet resulting in large $E_{\mathrm{T}}^{\text {miss }}$ :

- A reconstructed primary vertex with at least two associated tracks (with $p_{\mathrm{T}}>$ $0.4 \mathrm{GeV}$ ) is required [62]. This ensures that the recorded event is consistent with a proton-proton collision rather than a noise event.

- The highest- $p_{\mathrm{T}}$ jet must have a charge fraction $f_{\mathrm{ch}}=\sum p_{\mathrm{T}}^{\text {track,jet }} / p_{\mathrm{T}}^{\text {jet }}>0.02$, where $\sum p_{\mathrm{T}}^{\text {track,jet }}$ is the scalar sum of the transverse momenta of tracks associated with the primary vertex within a cone of radius $\Delta R=0.4$ around the jet axis, and $p_{\mathrm{T}}^{\text {jet }}$ is the transverse momentum of the jet as determined from calorimeter measurements. Furthermore, events are rejected if they contain any jet with an electromagnetic fraction $f_{\mathrm{em}}$ (fraction of the jet energy measured in the electromagnetic calorimeter) of less than 0.1 , or any jet in the pseudorapidity range $|\eta|<2$ with $f_{\mathrm{em}}>0.95$ and a charge fraction $f_{\mathrm{ch}} \leq 0.05$. These requirements suppress jets produced by cosmic rays or beam-background muons that interact in the hadronic calorimeter without corresponding signals in the electromagnetic calorimeter or the tracking detector.

- Additional selection criteria to reject events with significant detector noise and noncollision backgrounds are applied: events are rejected if any jet with $p_{\mathrm{T}}>20 \mathrm{GeV}$ and $|\eta|<4.5$ does not pass all of the additional quality criteria described in ref. [63].

- The leading jet has to be within $|\eta|<2$, and no more than two jets with $p_{\mathrm{T}}>30 \mathrm{GeV}$ and $|\eta|<4.5$ are allowed. Back-to-back dijet events are suppressed by requiring the sub-leading jet not to point in the direction of $\mathbf{p}_{\mathrm{T}}^{\text {miss }}:\left|\Delta \phi\left(\mathbf{p}_{\mathrm{T}}^{\text {miss }}, \mathbf{p}_{\mathrm{T}}^{\text {jet2}}\right)\right|>0.5$.

- An electronics failure affecting $20 \%$ of the data sample created a small dead region in the second and third layers of the electromagnetic calorimeter. Any event with the two leading jets inside the affected region and either of the two jets pointing in the 


\begin{tabular}{|c|c|c|c|c|}
\hline Signal regions & SR1 & $\mathrm{SR} 2$ & SR3 & SR4 \\
\hline Common requirements & \multicolumn{4}{|c|}{$\begin{array}{c}\text { Data quality }+ \text { trigger }+ \text { vertex }+ \text { jet quality }+ \\
\left|\eta^{\text {jet } 1}\right|<2.0+\left|\Delta \phi\left(\mathbf{p}_{\mathrm{T}}^{\text {miss }}, \mathbf{p}_{\mathrm{T}}^{\text {jet2 }}\right)\right|>0.5+N_{\text {jets }} \leq 2+ \\
\text { lepton veto }\end{array}$} \\
\hline$E_{\mathrm{T}}^{\mathrm{miss}}, p_{\mathrm{T}}^{\text {jet } 1}>$ & $120 \mathrm{GeV}$ & $220 \mathrm{GeV}$ & $350 \mathrm{GeV}$ & $500 \mathrm{GeV}$ \\
\hline
\end{tabular}

Table 3. Definition of the four overlapping signal regions SR1-SR4. Data quality, trigger, vertex, and jet quality refer to the selection criteria discussed in the main text.

direction of $E_{\mathrm{T}}^{\text {miss }}$ is removed from the sample to avoid fake signals. This condition removes only a few percent of the affected subset of the data.

- Events are required to have no SR-electron or SR-muon. In the background control regions, electrons and muons are explicitly selected. The electron and muon selection criteria in the signal and control regions are given in section 3 .

Although the results of this analysis are interpreted in terms of the ADD model and WIMP pair production, the event selection criteria have not been tuned to maximise the sensitivity to any particular BSM scenario. To maintain sensitivity to a wide range of BSM models, four sets of overlapping kinematic selection criteria, designated as SR1 to SR4, differing in the values of the requirements for $E_{\mathrm{T}}^{\text {miss }}$ and leading jet $p_{\mathrm{T}}$, are defined (table 3 ). Note that the requirement on the leading jet $p_{\mathrm{T}}$ is the same as that on $E_{\mathrm{T}}^{\mathrm{miss}}$ for all signal regions. In comparison with the previous ATLAS monojet search [16], the veto on additional jets is less stringent, allowing a second jet in the event thereby reducing systematic uncertainties from ISR/FSR (see section 6) and increasing signal selection efficiencies. The signal region with the lowest $E_{\mathrm{T}}^{\text {miss }}$ requirement (SR1) is chosen such that the $E_{\mathrm{T}}^{\text {miss }}$ trigger is nearly $100 \%$ efficient. The signal region with the highest $E_{\mathrm{T}}^{\mathrm{miss}}$ requirement (SR4) is chosen so that there remain enough events in data control samples to validate $\mathrm{MC}$ predictions and estimate SR backgrounds in a data-driven way.

\section{Background estimation}

A number of SM processes can pass the monojet kinematic selection criteria described above. These backgrounds include, in decreasing order of importance: $Z$ and $W$ boson plus jets production, single or pair production of top quarks, multijet production, cosmic-ray and beam-background muons ${ }^{3}$ (collectively referred to as non-collision background [64]), and di-boson production $(W W, W Z, Z Z)$. The dominant $Z / W$ plus jets backgrounds are estimated using control regions in the data with corrections that account for differences between the selection requirements of the signal and control regions (see section 5.1). The multijet and non-collision backgrounds are also estimated from data (see sections 5.2 and 5.3, respectively) while the di-boson and top-quark backgrounds are obtained from MC simulations.

\footnotetext{
${ }^{3}$ Originating either from protons going in the direction of the experiment and hitting the LHC collimation system or gas molecules in the beam-pipe near the ATLAS interaction point.
} 


\subsection{Backgrounds from $Z / W+$ jets}

The dominant background process for this search is irreducible and consists of the production of $Z$ bosons in association with jets, where the $Z$ decays to two neutrinos. A substantial source of reducible background is SM $W$ boson plus jets production where the $W$ decays to a charged lepton ( $\tau, e$, or $\mu$ in decreasing order of importance) and a neutrino. This process leads to a monojet final state if the lepton is outside the detector acceptance, is missed because of reconstruction inefficiencies or if a hadronic $\tau$ decay is reconstructed as a single jet. The $Z$ and $W$ boson plus jets backgrounds, collectively referred to in the following as electroweak backgrounds, are determined in a data-driven way:

1. Control regions are defined by explicitly selecting electrons or muons while keeping the same jet and $E_{\mathrm{T}}^{\text {miss }}$ selection criteria as in the signal regions. In a first step, samples enriched with four processes containing electrons or muons are separately selected with dedicated selection requirements: $W \rightarrow e \nu+$ jets, $W \rightarrow \mu \nu+$ jets, $Z \rightarrow$ $e^{+} e^{-}+$jets, $Z \rightarrow \mu^{+} \mu^{-}+$jets. In a second step, the jet and $E_{\mathrm{T}}^{\text {miss }}$ selection criteria as in the signal regions are imposed. Corrections are made for contamination of these control samples from processes other than $Z$ or $W$ decays.

2. Correction factors are then applied to account for differences in trigger and kinematic selection criteria between the control and signal regions. The control-to-signal region transfer factors, which are multiplied by the number of control-region events obtained in the previous step to yield the background estimate, are obtained using both data and simulation (see below).

In this approach, the modelling of the jet and $E_{\mathrm{T}}^{\text {miss }}$ kinematics of the electroweak backgrounds is obtained directly from data. Simulations are therefore used only for quantities related to the electron and muon selection criteria, and only through ratios where systematic uncertainties related to the jet and $E_{\mathrm{T}}^{\text {miss }}$ selection criteria of the control regions cancel. Theoretical uncertainties normally associated with MC estimates are significantly reduced; only distributions related to the electron and muon selection criteria have to be well modelled in the simulations. Further experimental uncertainties that impact the background prediction, such as the jet energy scale (JES) and resolution (JER) [55], the trigger efficiency, and the luminosity measurement [36, 37], are minimised by this approach.

The control regions are expected to have no contamination from BSM signals that would normally pass the monojet event selection criteria. They are chosen such that they are dominated by $Z$ and $W$ decays with reconstructed electrons or muons. The selection criteria follow closely those used in $Z$ and $W$ cross-section measurements [65]. The kinematic selection criteria on $E_{\mathrm{T}}^{\text {miss }}$ and jet $p_{\mathrm{T}}$ of the signal regions are also applied. Therefore, each CR is split into four subsets corresponding to the four signal regions. Four visible decay modes are used for the background estimates: $W \rightarrow e \nu+$ jets, $W \rightarrow$ $\mu \nu+$ jets, $Z \rightarrow e^{+} e^{-}+$jets, $Z \rightarrow \mu^{+} \mu^{-}+$jets. Based on these, all contributions to the signal regions from $Z$ and $W$ decay modes are estimated with the same method (except for $Z \rightarrow e^{+} e^{-}+$jets, which is found to be negligible in the signal regions because both $e^{+}$ and $e^{-}$would have to be missed in the event selection). In total, six background processes 


\begin{tabular}{|l|llll|}
\hline SR process & $Z \rightarrow \nu \bar{\nu}+$ jets & $\begin{array}{l}W \rightarrow \tau \nu+\text { jets } \\
W \rightarrow \mu \nu+\text { jets }\end{array}$ & $W \rightarrow e \nu+$ jets & $\begin{array}{l}Z \rightarrow \tau^{+} \tau^{-}+\text {jets } \\
Z \rightarrow \mu^{+} \mu^{-}+\text {jets }\end{array}$ \\
\hline & $W \rightarrow e \nu+$ jets & & \\
CR process & $W \rightarrow \mu \nu+$ jets & $W \rightarrow \mu \nu+$ jets & $W \rightarrow e \nu+$ jets & $Z \rightarrow \mu^{+} \mu^{-}+$jets \\
& $Z \rightarrow e^{+} e^{-}+$jets & & \\
& $Z \rightarrow \mu^{+} \mu^{-}+$jets & & \\
\hline
\end{tabular}

Table 4. Overview of processes in the control regions (CR) used to estimate background contributions to processes in the signal regions (SR).

in each signal region are predicted based on four control region processes, as detailed in table 4 . Note that the $W \rightarrow \tau \nu+$ jets background in the signal regions, where the $\tau$ lepton decays hadronically, can safely be estimated from $W \rightarrow \mu \nu+$ jets in control regions, since the jet and $E_{\mathrm{T}}^{\text {miss }}$ kinematics are the same. In both cases the leading jet is from radiation and recoils against the neutrino from the $W$ decay. The hadronic $\tau$ decay results in a jet that is either below the jet threshold of $30 \mathrm{GeV}$ or above this threshold but still sub-dominant compared to the leading jet from radiation.

For control regions that include processes with electrons, an electron trigger is used that requires a correction to account for differences in efficiency and acceptance compared to the $E_{\mathrm{T}}^{\text {miss }}$ trigger used for the signal regions. ${ }^{4}$ This different treatment is required because the energy deposited by electrons is included in the $E_{\mathrm{T}}^{\text {miss }}$ measurement at trigger level and results in the selection of a different kinematic region than that of the signal regions, which exclude electrons. Muons, however, do not deposit large amounts of energy in the calorimeters and are not explicitly included in the $E_{\mathrm{T}}^{\text {miss }}$ trigger. The specific selection criteria for the four control region processes are given in the following:

- $W \rightarrow e \nu+j e t s:$ events are selected using electron triggers with thresholds of 20 or $22 \mathrm{GeV}$ depending on the data-taking period. The CR-electron definition is used (see section 3 ) and exactly one electron with a $p_{\mathrm{T}}$ of at least $25 \mathrm{GeV}$ is required. Events with additional electrons or muons are discarded. All triggers used are fully efficient above the chosen $p_{\mathrm{T}}$ cut value. If an object is reconstructed as both an electron and a jet, the jet is removed from the reconstructed jet collection if $\Delta R(e$, jet $)<0.2$ while the electron is kept. To further improve the $W$ purity, $E_{\mathrm{T}}^{\text {miss }}>25 \mathrm{GeV}$ and $40<m_{\mathrm{T}}<100 \mathrm{GeV}$ are required. $m_{\mathrm{T}}$ is the transverse mass and it is defined as $m_{\mathrm{T}}=\sqrt{2 p_{\mathrm{T}} E_{\mathrm{T}}^{\text {miss }}\left(1-\cos \Delta \phi\left(\mathbf{p}_{\mathrm{T}}^{\text {lepton }}, \mathbf{p}_{\mathrm{T}}^{\text {miss }}\right)\right)}$, using the $p_{\mathrm{T}}$ of the lepton (electron or muon). $\Delta \phi$ is the angle between the lepton and the missing transverse momentum vector. As mentioned earlier, the same selection criteria on jet $p_{\mathrm{T}}$ and $E_{\mathrm{T}}^{\text {miss }}$ are applied in the control regions as in the signal regions (see table 3 ). However, when

\footnotetext{
${ }^{4}$ Note that the acceptance is defined as the ratio of the number of events within the detector volume that pass analysis requirements to the number of originally simulated events. The efficiency is defined as the ratio of the number of events within the detector volume at reconstruction level to that at the original simulation level.
} 
the $W \rightarrow e \nu+$ jets CR is used to estimate the contribution of $Z \rightarrow \nu \bar{\nu}+$ jets to each SR, a special CR is defined where $E_{\mathrm{T}}^{\text {miss }}$ is substituted by $E_{\mathrm{T}}^{\mathrm{miss}, \phi}$ to mimic the kinematics of the decay of the $Z$ boson to two undetected neutrinos. The standard calorimeterbased $E_{\mathrm{T}}^{\text {miss }}$ is used for the CR to estimate the $W \rightarrow e \nu+$ jets contribution to the SRs.

- $W \rightarrow \mu \nu+j e t s$ : events have to pass the same inclusive $E_{\mathrm{T}}^{\text {miss }}$ trigger that is used for the signal regions. Exactly one CR-muon (see section 3) is required, and events with additional electrons or muons are rejected. Cuts on the transverse mass and missing transverse momentum are applied to improve the purity for $W$ 's: $m_{\mathrm{T}}>40 \mathrm{GeV}$, $E_{\mathrm{T}}^{\text {miss }, \mu}>25 \mathrm{GeV}$. Note that the $E_{\mathrm{T}}^{\text {miss }}$ that includes the muon contribution, $E_{\mathrm{T}}^{\mathrm{miss}, \mu}$, is used for the $W$-specific selection cuts. For each kinematic region listed in table 3 , the standard calorimeter-based $E_{\mathrm{T}}^{\mathrm{miss}}$ is used to define the CRs for the estimates of both $Z \rightarrow \nu \bar{\nu}+$ jets and $W \rightarrow \mu \nu+$ jets in the corresponding SR.

- $Z \rightarrow e^{+} e^{-}+$jets: electron triggers are used in this channel. Exactly two opposite-sign electrons are required and events with additional electrons or muons are discarded. The selected electrons have to satisfy $p_{\mathrm{T}}>25$ (20) $\mathrm{GeV}$ for the leading (sub-leading) electron. Jet-electron overlap removal is performed as described above for $W \rightarrow$ $e \nu+$ jets. Finally, to enhance the fraction of $Z$ 's, an invariant mass requirement of $66<m_{e^{+} e^{-}}<116 \mathrm{GeV}$ is applied. $E_{\mathrm{T}}^{\text {miss, } \mathscr{\phi}}$ is used to define these CRs, which are used to estimate the $Z \rightarrow \nu \bar{\nu}+$ jets contribution to the SRs.

- $Z \rightarrow \mu^{+} \mu^{-}+j e t s$ : the inclusive $E_{\mathrm{T}}^{\text {miss }}$ trigger is used in this channel. Exactly two opposite-sign CR-muons (defined in section 3) are required and events with additional electrons or muons are rejected. An invariant mass of $66<m_{\mu^{+} \mu^{-}}<116 \mathrm{GeV}$ is required to select $Z$ candidates. The signal-region selection criteria are then applied on the calorimeter-based $E_{\mathrm{T}}^{\text {miss }}$, for the $Z \rightarrow \nu \bar{\nu}+$ jets, $Z \rightarrow \tau^{+} \tau^{-}+$jets, $Z \rightarrow \mu^{+} \mu^{-}+$jets estimates.

Using the control regions defined above, the background contribution to the signal regions for each combination of $\mathrm{CR}$ and SR processes mentioned in table 4 is derived using:

$$
\begin{aligned}
N_{\mathrm{SR}}^{\text {predicted }} & =\left(N_{\mathrm{CR}}^{\text {Data }}-N_{\mathrm{CR}}^{\mathrm{Bkg}}\right) \cdot C \cdot T \\
& =\left(\left(N_{\mathrm{CR}}^{\text {Data }}-N_{\mathrm{CR}}^{\mathrm{multijet}}\right) \cdot\left(1-f_{\mathrm{EW}}\right)\right) \times \frac{\epsilon_{E_{\mathrm{T}}^{\text {miss }}}^{\text {trig }} \cdot \mathcal{L}_{E_{\mathrm{T}}^{\mathrm{miss}}}}{A_{\ell} \cdot \epsilon_{\ell} \cdot \epsilon_{Z / W} \cdot \epsilon_{\ell}^{\mathrm{trig}} \cdot \mathcal{L}_{\ell}} \times \frac{N_{\mathrm{SR}}^{\mathrm{MC}}}{N_{\text {jet} / \mathrm{E}_{\mathrm{T}}^{\mathrm{miss}}}^{\mathrm{MC}}} .
\end{aligned}
$$

Data in the control regions are corrected for contamination arising from other sources (summarised as $N_{\mathrm{CR}}^{\mathrm{Bkg}}$ in the first line). Correction factors $(C)$ based on MC simulation and data are applied together with the transfer factor $T$ to obtain the number of background events, $N_{\mathrm{SR}}^{\text {predicted }}$, predicted in the signal region. The terms appearing in the second line of equation 5.1 are:

- $N_{\mathrm{CR}}^{\mathrm{Data}}$ and $N_{\mathrm{CR}}^{\mathrm{multijet}}$ are the number of data and multijet events in the control region, respectively. To estimate the multijet contamination of control regions by processes 
with identified electrons, the selection cuts, in particular the isolation cuts, are varied. The fake rate in those regions is extracted from data using real and fake electron efficiencies determined from samples enriched in electrons and jets. Using this estimate, the multijet contamination is predicted to account for $1-2 \%$ of the events in the $W \rightarrow e \nu+$ jets electron control region when predicting the $Z \rightarrow \nu \bar{\nu}+$ jets contribution to the SRs. For other control regions containing electrons or muons, the multijet contamination is found to be negligible using similar techniques.

- $f_{\mathrm{EW}}$ is the estimated fraction of events, after multijet corrections, due to contamination of the control region by other electroweak or other SM processes. This contamination is due to top-quark and di-boson decays as well as decays of $Z$ or $W$ bosons to leptons of a flavour other than the one selected for that control region. The contribution of this contamination is obtained from MC simulation and is about $2 \%$ for $Z$ bosons, and $10 \%$ for $W$ bosons. The top-quark and di-boson contribution is negligible. As explained above, using ratios of MC estimates ( $f_{\mathrm{EW}}$ in this case) is advantageous as it leads to cancellations of systematic uncertainties.

- $A_{\ell}$ and $\epsilon_{\ell}$ are the lepton acceptance obtained from simulation and the identification efficiency obtained from data $[56,57]$, respectively.

- $\epsilon_{Z / W}$ are the efficiencies for the $Z$ or $W$ boson selection criteria obtained from simulation. The factors $A_{\ell}, \epsilon_{\ell}$, and $\epsilon_{Z / W}$ correct for the fact that leptons and $Z / W$ bosons are required only in the control regions.

- $\epsilon_{\ell}^{\text {trig }}$ and $\mathcal{L}_{\ell}$ are the electron trigger efficiency (obtained from data) and the corresponding luminosity associated with this trigger for the relevant control region. For muon control regions these factors do not apply because the signal-region trigger is used in the definition of the CR.

- $\epsilon_{E_{\mathrm{T}}^{\text {miss }}}^{\text {trig }}$ and $\mathcal{L}_{E_{\mathrm{T}}^{\text {miss }}}$ are the $E_{\mathrm{T}}^{\text {miss }}$ trigger efficiency (obtained from data) and the corresponding luminosity, and are only relevant for electron control regions where the electron trigger efficiency and luminosity $\left(\epsilon_{\ell}^{\text {trig }}\right.$ and $\left.\mathcal{L}_{\ell}\right)$ need to be corrected, accounting for the different triggers used in the definition of the signal and control regions.

- The transfer factor $T=\frac{N_{\mathrm{SR}}^{\mathrm{MC}}}{N_{\mathrm{jet} / \mathrm{E}_{\mathrm{T}}^{\mathrm{miss}}}^{\mathrm{m}}}$ is the ratio of simulated background events in the signal region (for example $Z \rightarrow \nu \bar{\nu}+$ jets) to simulated events of a control-region process (for example $W \rightarrow e \nu+$ jets) with only jet and $E_{\mathrm{T}}^{\text {miss }}$ related selection requirements applied. This term translates the number of observed events in the CR in the data to the predicted number of events in the signal region. Depending on the control region and the signal-region background component being determined, this factor can account for ratios of branching fractions, ratios of $W+$ jets to $Z+$ jets cross sections, and phase-space differences between the control and signal regions for a given source of background. 
The correction factors and electroweak background predictions in equation 5.1 are determined in bins of $E_{\mathrm{T}}^{\text {miss }}$ for the final background prediction, and in bins of the leading and sub-leading jet $p_{\mathrm{T}}$ for the jet- $p_{\mathrm{T}}$ plots in figure 2 .

\subsection{Multijet backgrounds}

Multijet events where one or more jets are severely mismeasured constitute a background that is not well modelled in the simulation. In order to measure this background from data, a sample is selected by applying all signal-region selection criteria except for the jet vetoes: A) either a second jet with $\left|\Delta \phi\left(\mathbf{p}_{\mathrm{T}}^{\text {miss }}, \mathbf{p}_{\mathrm{T}}^{\text {jet2 }}\right)\right|<0.5$ is required, B) or the third-jet veto is reversed by requiring three jets, $N_{\text {jet }}=3$, and missing transverse momentum to be aligned with the third jet: $\left|\Delta \phi\left(\mathbf{p}_{\mathrm{T}}^{\text {miss }}, \mathbf{p}_{\mathrm{T}}^{\text {jet3 }}\right)\right|<0.5$ and $\left|\Delta \phi\left(\mathbf{p}_{\mathrm{T}}^{\text {miss }}, \mathbf{p}_{\mathrm{T}}^{\text {jet} 2}\right)\right|>0.5$. These two samples are used to predict the multijet background from the resulting di- or trijet events. Contributions to these event samples from top-quark, and $Z$ or $W$ production are subtracted. The MC simulation is used for the top-quark contribution. For $Z$ and $W$, MC estimates normalised to data are used for the subtraction. The multijet background is then estimated by fitting a straight line to the second or third jet $p_{\mathrm{T}}$ distributions in events passing the two selection criteria (A) and B)) and then extrapolating the fit below a $p_{\mathrm{T}}$ of $30 \mathrm{GeV}$. For this value of the transverse momentum, the jets fall below the threshold and can pass the monojet selection criteria. Note that the number of trijet events where both sub-leading jets are mismeasured, and fall below the jet threshold, is negligible compared to the case where either the second or the third jet is lost. The resulting background estimates are given in table 6 . They are at most $1 \%$ of the total background predicted for SR1-SR3, and are negligible for SR4.

\subsection{Non-collision backgrounds}

Non-collision backgrounds in the signal regions are estimated using a dedicated algorithm that identifies beam-background muons that go through the detector along the direction of the beams. The algorithm selects through-going muons based on timing information obtained from the muon chambers in the forward regions. It combines this information with calorimeter energy clusters by matching them in $\phi$. Unpaired proton bunches, where the bunch from one of the proton beams is empty, are used to determine identification efficiencies of the algorithm for beam-background and cosmic-ray muons. These efficien-

cies $\left(\epsilon_{\text {tag }}^{\text {non-coll. }}\right)$, typically $20-50 \%$, are used together with the number of beam-background ( halo) candidates found in the signal regions, to predict the level of non-collision background $\left(N_{\text {non-coll. }}=N_{\text {halo }} / \epsilon_{\text {tag }}^{\text {non-coll. }}\right)$. More details of this background component are given in ref. [64]. It contributes mainly in SR1 and SR2 at less than 1\%. The predictions are given in table 6 .

\subsection{Systematic uncertainties on background estimates}

The dominant systematic uncertainties associated with the electroweak background estimates are on JES and $E_{\mathrm{T}}^{\text {miss }}$, as well as theoretical uncertainties on the shape of $W$ kinematic distributions and the ratio of $Z$ and $W$ plus jets production cross sections. The latter theoretical uncertainty is relevant because background predictions from $W$ control 
regions are also used to estimate $Z \rightarrow \nu \bar{\nu}+$ jets contributions to the signal region. Additional systematic uncertainties are due to the muon momentum scale and resolution, the data-driven scale factors to equalise lepton trigger and reconstruction efficiencies in simulations and data, statistical uncertainties associated with the limited size of MC samples, the subtraction of the electroweak contamination $\left(f_{\mathrm{EW}}\right)$, and, in the electron control regions, the multijet contamination. The uncertainties from pile-up variations are found to be negligible, as are those from the electron energy scale, resolution, and JER.

The systematic uncertainties associated with the small multijet background are estimated to be $100 \%$. They are obtained by changing the fit range for the $p_{\mathrm{T}}$ extrapolation and varying the scale factors for the $Z$ and $W$ background prediction by $10 \%$. All variations are within a factor of two of the central predictions.

Systematic uncertainties on the non-collision background are $10 \%$. This estimate corresponds to the average fraction of unpaired proton bunches that are used to determine $\epsilon_{\mathrm{tag}}^{\text {non-coll. }}$, and that are close (separated by $25 \mathrm{~ns}$ in time) to an unpaired bunch in the opposite beam. Such configurations may lead to double counting in the efficiency estimate, and their total contribution is hence considered as an uncertainty.

The JES and JER uncertainties are evaluated using a combination of data-driven and MC-based techniques [55]. These methods take into account the variation of the uncertainty with jet $p_{\mathrm{T}}$ and $\eta$, and the presence of nearby jets. The $E_{\mathrm{T}}^{\text {miss }}$ uncertainty is derived from the JES and JER uncertainties by propagating the relative jet-level variations to the calorimeter cluster based $E_{\mathrm{T}}^{\mathrm{miss}}$. Since ratios are used to extrapolate from the control regions to the signal regions, the effects of these uncertainties tend to cancel.

Theoretical uncertainties on $Z$ and $W$ production and the shape of $W$ kinematic distributions are evaluated by comparing background estimates using kinematic $Z / W$ distributions from different generators (ALPGEN and SHERPA). Uncertainties on $f_{\mathrm{EW}}$ are derived by comparing ALPGEN to PYTHIA [66], but also by taking into account JES and lepton-scale uncertainties. The full difference is taken as systematic uncertainty in all cases.

Systematic and statistical uncertainties on all background estimates are given in table 5. The contribution from lepton scale factors is the quadratic sum of electron and muon uncertainties. The uncertainties from di-boson, top-quark, multijet, and non-collision backgrounds are summed in quadrature. A 20\% uncertainty is assigned for the di-boson and top-quark MC-based estimates. This value is dominated by the JES uncertainty (16\%), but also takes into account uncertainties of the trigger efficiency, luminosity measurement, and lepton identification uncertainties.

\subsection{Background summary and additional checks}

An overview of all backgrounds is given in table 6 (cf. table 4 for the definition of the control regions). The final $Z \rightarrow \nu \bar{\nu}+$ jets predictions are estimated from a combination of the predictions of the four control regions. The combination is the error-weighted average calculated taking into account correlations of uncertainties. The $Z \rightarrow \nu \bar{\nu}+$ jets prediction is dominated by the $W$ control-region estimates and based on the assumption that the ratio of $Z+$ jets to $W+$ jets cross sections is well modelled in the simulation. This assumption is supported by dedicated measurements [66], albeit for smaller jet momenta than the 


\begin{tabular}{|l|llll|}
\hline Source & SR1 & SR2 & SR3 & SR4 \\
\hline JES/JER/ $E_{\mathrm{T}}^{\text {miss }}$ & 1.0 & 2.6 & 4.9 & 5.8 \\
MC $Z / W$ modelling & 2.9 & 2.9 & 2.9 & 3.0 \\
MC statistical uncertainty & 0.5 & 1.4 & 3.4 & 8.9 \\
$1-f_{\mathrm{EW}}$ & 1.0 & 1.0 & 0.7 & 0.7 \\
Muon scale and resolution & 0.03 & 0.02 & 0.08 & 0.61 \\
Lepton scale factors & 0.4 & 0.5 & 0.6 & 0.7 \\
Multijet BG in electron CR & 0.1 & 0.1 & 0.3 & 0.6 \\
Di-boson, top, multijet, non-collisions & 0.8 & 0.7 & 1.1 & 0.3 \\
\hline Total systematic uncertainty & 3.4 & 4.4 & 6.8 & 11.1 \\
Total data statistical uncertainty & 0.5 & 1.7 & 4.3 & 11.8 \\
\hline
\end{tabular}

Table 5. Relative systematic uncertainties for all signal regions (in percent). Individual contributions are summed in quadrature to derive the total numbers. The MC statistical uncertainty is included in the total systematic uncertainty.

ones used in SR2 to SR4. The theoretical uncertainty on the ratio of $Z$ to $W$ cross sections is included in the uncertainty derived from comparisons of different $\mathrm{MC}$ generators discussed above.

The electroweak background estimate, which relies on an exclusive $W$ or $Z$ selection in the control regions, is compared to two alternative correlated methods. In the first of these, which was the main method used in the previous ATLAS monojet search [16], an inclusive control region is defined by only inverting the lepton veto while keeping all other selection criteria the same as in the signal regions. No additional $Z$ - or $W$-specific invariant or transverse mass selection criteria are applied, thereby yielding a mixed control sample dominated by $W$ and $Z$ bosons. The resulting background predictions are found to be consistent with those of the default method. The second alternative modifies the lepton definition in the control regions. Instead of applying lepton selection criteria in control regions that are more stringent than those of the signal regions, a modified exclusive control region is defined. The selection criteria include less stringent lepton definitions where the lepton veto cuts of the signal region are simply inverted, and dedicated $Z$ or $W$ selection criteria are used. These background predictions are also found to be consistent with the default method.

Distributions from all four visible decay modes used to determine the background in SR1 are shown in figure 1. The distributions are obtained by applying the exclusive $Z$ and $W$ selection criteria plus SR1 kinematic cuts on $E_{\mathrm{T}}^{\mathrm{miss}}$ and jets, as well as vetoes on additional electrons or muons. It should be noted that shape differences in the $E_{\mathrm{T}}^{\text {miss }}$ distributions between data and $\mathrm{MC}$ are irrelevant for an accurate background prediction in the signal regions, because the $E_{\mathrm{T}}^{\text {miss }}$ distribution obtained from control-region data is used directly to predict the backgrounds in the signal regions. Distributions of variables that are subject to MC-based efficiency or acceptance corrections, namely those involving electrons or muons, need to agree in shape between data and MC (see figure 1, where good shape agreement is found for the leading electron and muon $p_{\mathrm{T}}$ distributions). 


\begin{tabular}{|l|cccc|}
\hline & SR1 & SR2 & SR3 & SR4 \\
\hline$Z \rightarrow \nu \bar{\nu}+$ jets & $63000 \pm 2100$ & $5300 \pm 280$ & $500 \pm 40$ & $58 \pm 9$ \\
$W \rightarrow \tau \nu+$ jets & $31400 \pm 1000$ & $1853 \pm 81$ & $133 \pm 13$ & $13 \pm 3$ \\
$W \rightarrow e \nu+$ jets & $14600 \pm 500$ & $679 \pm 43$ & $40 \pm 8$ & $5 \pm 2$ \\
$W \rightarrow \mu \nu+$ jets & $11100 \pm 600$ & $704 \pm 60$ & $55 \pm 6$ & $6 \pm 1$ \\
$t \bar{t}+$ single $t$ & $1240 \pm 250$ & $57 \pm 12$ & $4 \pm 1$ & - \\
Multijets & $1100 \pm 900$ & $64 \pm 64$ & $8_{-8}^{+9}$ & - \\
Non-coll. Background & $575 \pm 83$ & $25 \pm 13$ & - & - \\
$Z / \gamma^{*} \rightarrow \tau \tau+$ jets & $421 \pm 25$ & $15 \pm 2$ & $2 \pm 1$ & - \\
Di-bosons & $302 \pm 61$ & $29 \pm 5$ & $5 \pm 1$ & $1 \pm 1$ \\
$Z / \gamma^{*} \rightarrow \mu \mu+$ jets & $204 \pm 19$ & $8 \pm 4$ & - & - \\
\hline Total Background & $124000 \pm 4000$ & $8800 \pm 400$ & $750 \pm 60$ & $83 \pm 14$ \\
Events in Data $\left(4.7 \mathrm{fb}^{-1}\right)$ & 124703 & 8631 & 785 & 77 \\
\hline$\sigma_{\text {vis }}^{\text {obs }}$ at $90 \%[\mathrm{pb}]$ & 1.63 & 0.13 & 0.026 & 0.0055 \\
$\sigma_{\text {vis }}^{\exp }$ at $90 \%[\mathrm{pb}]$ & 1.54 & 0.15 & 0.020 & 0.0064 \\
$\sigma_{\text {vis }}^{\text {obs }}$ at $95 \%[\mathrm{pb}]$ & 1.92 & 0.17 & 0.030 & 0.0069 \\
$\sigma_{\text {vis }}^{\exp }$ at $95 \%[\mathrm{pb}]$ & 1.82 & 0.18 & 0.024 & 0.0079 \\
\hline
\end{tabular}

Table 6. Overview of predicted SM background and observed events in data for $4.7 \mathrm{fb}^{-1}$ for each of the four signal regions. The total uncertainty quoted is the quadratic sum of statistical and systematic uncertainties. Observed and expected $90 \%$ and $95 \%$ CL upper limits on the non-SM contribution to all signal regions are also given in terms of limits on visible cross sections $\left(\sigma_{\text {vis }} \equiv\right.$ $\sigma \times A \times \epsilon)$. The $90 \%$ CL upper limits are given to facilitate comparisons with other experiments.

\section{Results and interpretation}

The SM predictions are found to be consistent with the number of observed events in data for all signal regions considered. Comparisons of the SM predictions to the measured $E_{\mathrm{T}}^{\text {miss }}$ and leading and sub-leading jet $p_{\mathrm{T}}$ distributions are shown for SR1 and SR4 in figure 2. For illustration, the figures also contain simulated signal distributions for ADD and WIMP models added to the total background. Agreement both in the shape and the overall normalisation between SM predictions and data is observed in all cases. To facilitate comparisons with other experiments both $90 \%$ and the more conventional $95 \%$ confidence level (CL) upper limits are produced. These limits are on the visible cross section defined as cross section times acceptance and efficiency $(\sigma \times A \times \epsilon)$ and they are based on the modified frequentist $C L_{\mathrm{s}}$ prescription [67]. The limits are derived by comparing the probabilities, based on Poisson distributions, that the observed number of events is compatible with the SM and the SM-plus-signal expectations. The mean values of the Poisson distributions are determined by the signal prediction, plus contributions from background 

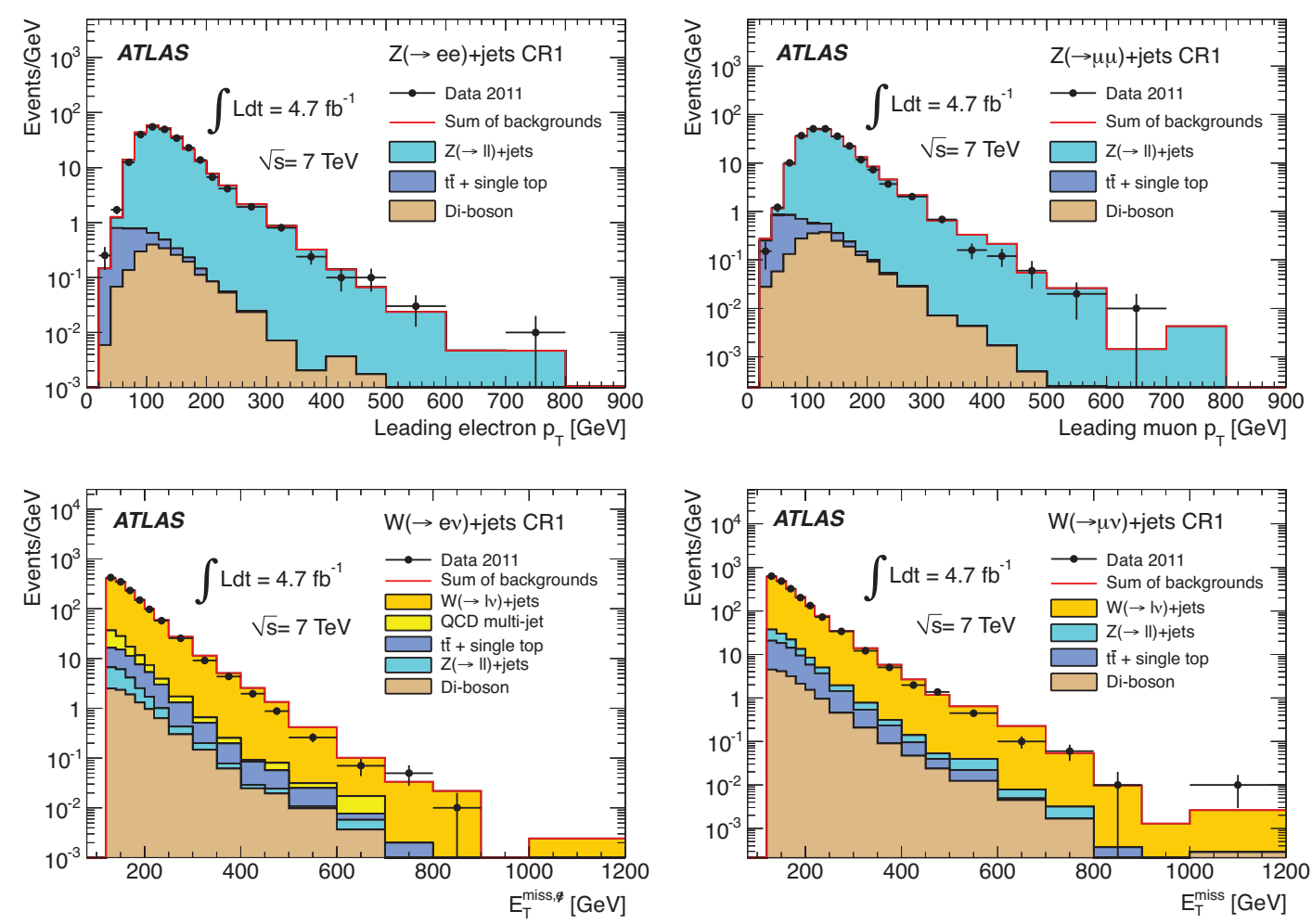

Figure 1. Kinematic distributions in the control regions corresponding to SR1 (labelled CR1) are shown. The upper row is the leading electron and muon $p_{\mathrm{T}}$ distribution for $Z \rightarrow e^{+} e^{-}+$jets (left) and $Z \rightarrow \mu^{+} \mu^{-}+$jets (right) and shows distributions after SR1 cuts on jets and $E_{\mathrm{T}}^{\mathrm{miss}}$. The lower row is the missing transverse momentum distribution $E_{\mathrm{T}}^{\text {miss, },}$ for $W \rightarrow e \nu+$ jets (left) and $E_{\mathrm{T}}^{\text {miss }}$ for $W \rightarrow \mu \nu+$ jets (right) also after SR1 jet and $E_{\mathrm{T}}^{\text {miss }}$ cuts.

processes extrapolated from the CRs to each SR. The number of events is integrated over the whole SR. Expected limits are obtained by repeating the analysis with pseudo-data obtained from Monte Carlo simulations. The distributions of the simulated probabilities for many pseudo-experiments allow $\pm 1 \sigma$ bands to be plotted for the expected values. Systematic uncertainties (and their correlations) associated with SM backgrounds and the integrated luminosity are taken into account via nuisance parameters using a profile likelihood technique [68]. The nuisance parameters are assumed to be Gaussian distributed in the likelihood fit. The resulting visible cross-section limits, which apply for any source of BSM events, are summarised in table 6. Typical efficiencies of selection criteria related to jets and $E_{\mathrm{T}}^{\text {miss }}$ of $\epsilon \sim 83 \%$ are found in simulated $Z \rightarrow \nu \bar{\nu}+$ jets, WIMP or ADD samples. Typical acceptances are given in table 7 . The negative search results are interpreted in terms of limits on ADD and WIMP model parameters in the following sections.

\subsection{Large extra dimensions}

Experimental and theoretical systematic uncertainties that affect the ADD signal are considered in order to set limits on the model parameters. The experimental uncertainties on JES, JER, and $E_{\mathrm{T}}^{\text {miss }}$ are considered to be fully correlated with those obtained for the 

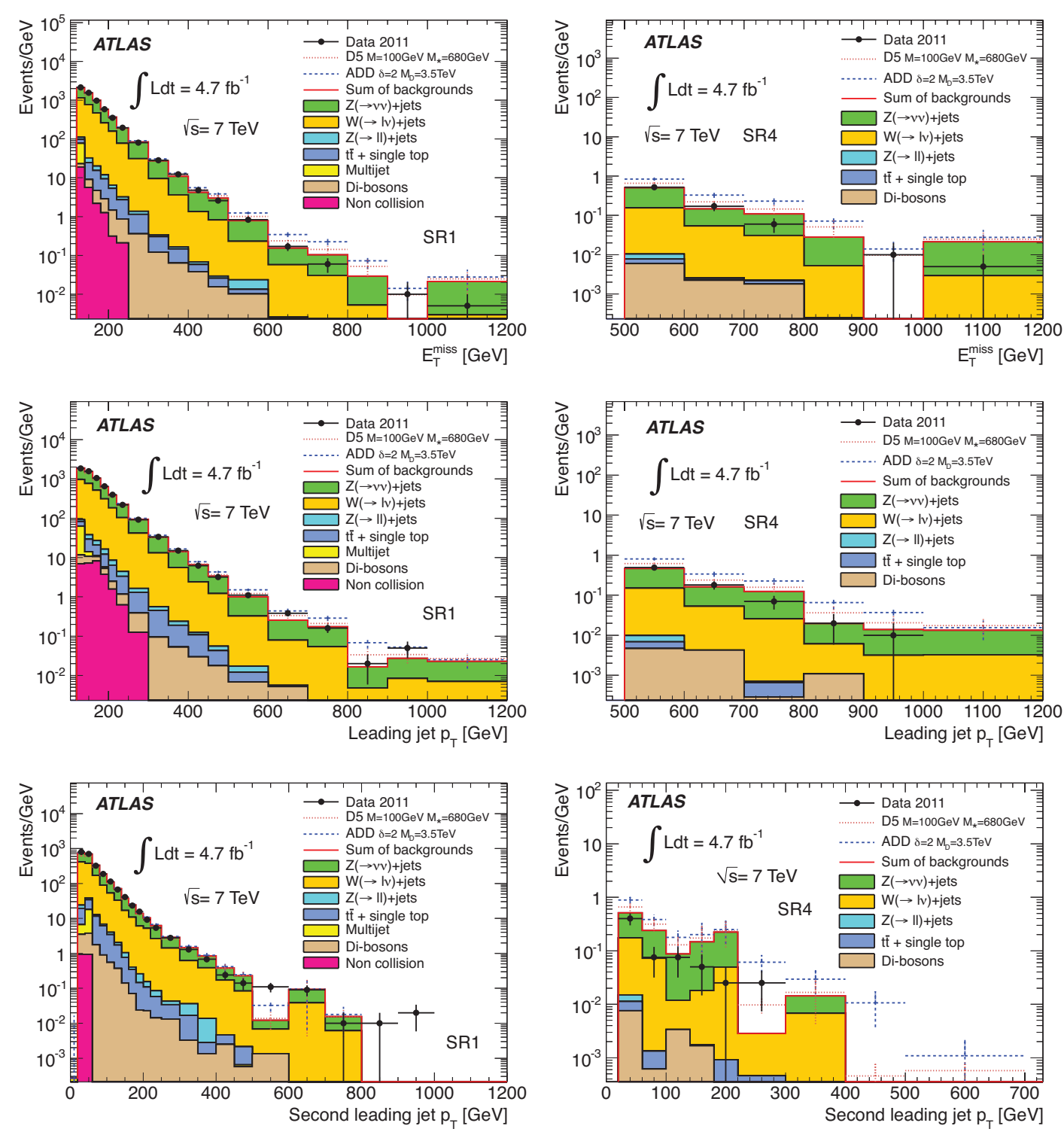

Figure 2. Kinematic distributions for signal regions SR1 on the left and SR4 on the right. Signal distributions for ADD and WIMP samples for cross sections equal to the excluded values are drawn as dashed lines on top of the predicted background distributions. The electroweak backgrounds (see equation 5.1) are determined in bins of the variable that is plotted.

background estimate. They range from $3-10 \%$ depending on the signal region and the number of extra dimensions. An additional $1 \%$ uncertainty on the trigger, and a $3.9 \%$ uncertainty on the luminosity are also considered for the signal simulation only. Theoretical uncertainties on the expected ADD signal are associated with the PDF set, ISR/FSR, and the factorisation and renormalisation scales. For the PDF uncertainties, the CTEQ6. 6 error sets are used, converted from $90 \%$ to $68 \%$ CL. They range from $4-14 \%$ on the product of signal cross section and acceptance $(\sigma \times A)$, depending on the number of extra dimensions. Uncertainties coming from the modelling of ISR/FSR are determined by varying 


\begin{tabular}{|l|cccc|}
\hline Sample & SR1 [\%] & SR2 [\%] & SR3 [ \% ] & SR4 [ \% ] \\
\hline$Z \rightarrow \nu \bar{\nu}+$ jets & $1.706 \pm 0.013$ & $0.159 \pm 0.004$ & $0.0170 \pm 0.0013$ & $0.0027 \pm 0.0005$ \\
ADD, $n=2$ & $30.9 \pm 0.2$ & $9.2 \pm 0.1$ & $2.60 \pm 0.07$ & $0.74 \pm 0.04$ \\
ADD, $n=3$ & $33.2 \pm 0.2$ & $11.7 \pm 0.1$ & $3.92 \pm 0.08$ & $1.18 \pm 0.05$ \\
ADD, $n=4$ & $34.3 \pm 0.2$ & $13.8 \pm 0.1$ & $4.97 \pm 0.09$ & $1.67 \pm 0.05$ \\
ADD, $n=5$ & $35.1 \pm 0.2$ & $14.5 \pm 0.1$ & $5.50 \pm 0.09$ & $2.00 \pm 0.06$ \\
ADD, $n=6$ & $35.0 \pm 0.2$ & $15.0 \pm 0.2$ & $6.01 \pm 0.10$ & $2.23 \pm 0.06$ \\
D1, $m_{\chi}=10 \mathrm{GeV}$ & $20.5 \pm 0.3$ & $3.3 \pm 0.1$ & $0.54 \pm 0.01$ & $0.09 \pm 0.01$ \\
D1, $m_{\chi}=1000 \mathrm{GeV}$ & $32.2 \pm 0.4$ & $10.3 \pm 0.2$ & $2.88 \pm 0.04$ & $0.79 \pm 0.02$ \\
D5, $m_{\chi}=10 \mathrm{GeV}$ & $30.4 \pm 0.4$ & $8.3 \pm 0.2$ & $2.04 \pm 0.03$ & $0.52 \pm 0.01$ \\
D5, $m_{\chi}=1000 \mathrm{GeV}$ & $36.2 \pm 0.4$ & $12.6 \pm 0.2$ & $4.14 \pm 0.05$ & $1.24 \pm 0.03$ \\
D9, $m_{\chi}=10 \mathrm{GeV}$ & $36.9 \pm 0.5$ & $12.9 \pm 0.3$ & $4.23 \pm 0.15$ & $1.31 \pm 0.08$ \\
D9, $m_{\chi}=1000 \mathrm{GeV}$ & $37.6 \pm 0.5$ & $13.9 \pm 0.3$ & $4.70 \pm 0.16$ & $1.68 \pm 0.09$ \\
D11, $m_{\chi}=10 \mathrm{GeV}$ & $30.3 \pm 0.4$ & $12.3 \pm 0.3$ & $4.57 \pm 0.15$ & $1.52 \pm 0.09$ \\
$\mathrm{D} 11, m_{\chi}=1000 \mathrm{GeV}$ & $33.7 \pm 0.5$ & $17.0 \pm 0.3$ & $7.56 \pm 0.20$ & $3.27 \pm 0.13$ \\
\hline
\end{tabular}

Table 7. Typical acceptances determined with MC simulations for the main background process $Z \rightarrow \nu \bar{\nu}+$ jets as well as for ADD and selected WIMP samples. For the $Z \rightarrow \nu \bar{\nu}+$ jets sample at least one parton with a minimum transverse momentum of $20 \mathrm{GeV}$ is required, for the ADD and WIMP samples it is at least one parton with a momentum of $80 \mathrm{GeV}$. The values are given in percent and errors are statistical only.

the simulation parameters of PYTHIA within a range that is consistent with experimental data [69]. The resulting uncertainties vary from about $3-14 \%$. The dominant theoretical systematic uncertainty affecting mostly the cross section rather than the acceptance is from the factorisation and renormalisation scales. Varying these scales between twice and half their default values, following common practice, results in 20-30\% uncertainties on $\sigma \times A .^{5}$

The visible cross sections predicted by the ADD generator for SR4 are shown for $n=2,4,6$ extra dimensions as a function of $M_{\mathrm{D}}$ on the left-hand side of figure 3 . Theoretical systematic uncertainties are shown as coloured bands around the cross-section curves. The 95\% CL expected and observed limits on the visible cross section $\sigma \times A \times \epsilon$ are shown as horizontal lines. The effect of restricting the simulated phase space to the kinematic region where the ADD effective field-theory implementation is valid is probed by evaluating the cross section after discarding events for which the parton centre-of-mass energy $\hat{s}>M_{\mathrm{D}}^{2}$. The amount by which the truncated cross sections differ from the full ones provides a measure for the reliability of the effective field theory. This difference increases from SR1 to SR4 and with the number of extra dimensions. While the model with $n=2$ extra dimensions is found to be insensitive to truncation effects for $M_{\mathrm{D}}$ values near the resulting

\footnotetext{
${ }^{5}$ Note that in ref. [16] the squared factorisation and renormalisation scales were varied between twice and half their default values.
} 

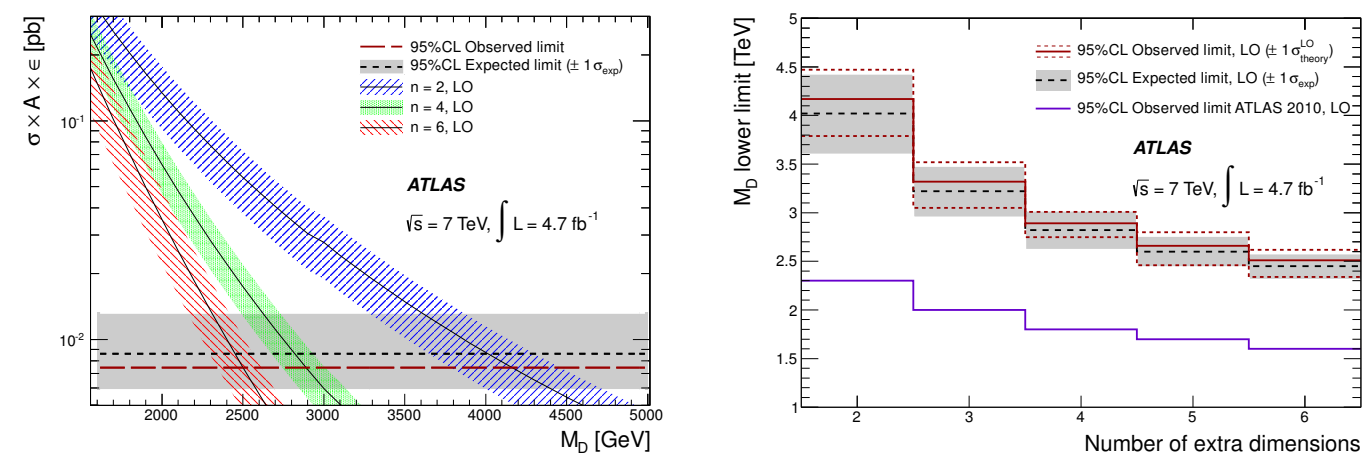

Figure 3. Left: visible cross sections in SR4 as a function of $M_{\mathrm{D}}$ as predicted by the effective ADD theory, for $n=2,4,6$ extra dimensions. The coloured bands correspond to the theoretical systematic uncertainties (PDF, ISR/FSR, scale). The horizontal lines are the expected and observed crosssection limits at $95 \%$ CL, taking into account experimental systematic uncertainties fully correlated between signal and background, as well as uncertainties on the luminosity estimate, trigger efficiency, and MC statistical uncertainties. The inclusion of signal uncertainties here increases the crosssection limits compared to those given in table 6, which exclude signal uncertainties. Right: $95 \%$ CL lower limits on $M_{\mathrm{D}}$ for different numbers of extra dimensions based on SR4. Observed and expected limits including all but the theoretical signal uncertainties are shown as solid and dashed lines, respectively. The grey $\pm 1 \sigma$ band around the expected limit is the variation expected from statistical fluctuations and experimental systematic uncertainties on SM and signal processes. The impact of the theoretical uncertainties is shown by the red small-dashed $\pm 1 \sigma$ limits. The previous ATLAS limit [16] is also shown for comparison.

\begin{tabular}{|c|cc|cc|cc|}
\hline \multirow{2}{*}{$n$} & \multicolumn{2}{|c|}{$M_{\mathrm{D}}[\mathrm{TeV}]$} & \multicolumn{2}{c|}{$R[\mathrm{pm}]$} & \multicolumn{2}{c|}{ Cross section truncation } \\
& LO & NLO & LO & NLO & LO & NLO \\
\hline 2 & 4.17 & 4.37 & $2.8 \times 10^{7}$ & $2.5 \times 10^{7}$ & $0.02 \%$ & $0.01 \%$ \\
3 & 3.32 & 3.45 & $4.8 \times 10^{2}$ & $4.5 \times 10^{2}$ & $1.9 \%$ & $1.3 \%$ \\
4 & 2.89 & 2.97 & 2.0 & 1.9 & $11.8 \%$ & $9.9 \%$ \\
5 & 2.66 & 2.71 & $7.1 \times 10^{-2}$ & $7.0 \times 10^{-2}$ & $29.5 \%$ & $27.2 \%$ \\
6 & 2.51 & 2.53 & $0.8 \times 10^{-2}$ & $0.8 \times 10^{-2}$ & $49.1 \%$ & $47.9 \%$ \\
\hline
\end{tabular}

Table 8. 95\% CL lower (upper) limits on $M_{\mathrm{D}}(R)$ for $n=2-6$ extra dimensions, using a dataset corresponding to $4.7 \mathrm{fb}^{-1}$ at $\sqrt{s}=7 \mathrm{TeV}$. These results are obtained using the selection criteria of SR4. All values correspond to the nominal observed limits excluding theoretical uncertainties in figure 3. The last two columns show the relative difference between the full cross sections and those of the truncated phase space $\left(\hat{s}<M_{\mathrm{D}}^{2}\right)$. The ADD cross sections are calculated at both LO and NLO, and the limits are derived from the full, not the truncated, phase space.

limits for all signal regions, $n=3,4,5$, and 6 extra dimensions show differences of $2 \%, 10 \%$, $30 \%$, and $50 \%$ between full and truncated cross sections for SR 4 and $M_{\mathrm{D}}$ values close to the actual limits (table 8). This demonstrates that the high energy and integrated luminosity used in this search allow to probe kinematic regions where the effective field-theory model is not entirely valid.

The $95 \%$ CL lower limits on $M_{\mathrm{D}}$ versus $n$ for the full phase space, not the truncated one, are shown for SR4 on the right-hand side of figure 3. The selection criteria of SR4 
provide the best expected limits and are therefore used here. Limits from SR1, SR2, SR3 are typically $35 \%, 15 \%, 5 \%$ worse, respectively. The expected and observed limits in figure 3 are produced taking all but the theoretical uncertainties into account. The grey $\pm 1 \sigma$ band around the expected limit shows the variation anticipated from statistical fluctuations and from experimental systematic uncertainties on background and signal processes. The impact of the theoretical uncertainties associated with PDFs, ISR/FSR, and factorisation and renormalisation scales is represented in the right-hand panel by dashed $\pm 1 \sigma$ lines on either side of the observed limit. The resulting limit is taken as the observed line excluding theoretical uncertainties. ${ }^{6}$ All limits from SR4 are summarised in table 8, where the lower (upper) limits on $M_{\mathrm{D}}(R)$ are shown for cross sections calculated at LO and NLO. The $K$-factors (defined as $\sigma_{\mathrm{NLO}} / \sigma_{\mathrm{LO}}$ ) for $n=2,3,4,5,6$ extra dimensions are 1.20, 1.20, $1.17,1.13,1.09$, respectively, and have been derived for the selection criteria of SR4 by the authors of ref. [49]. $M_{\mathrm{D}}$ values below 4.17 (4.37) TeV for $n=2$ and 2.51 (2.53) $\mathrm{TeV}$ for $n=6$ are excluded at $95 \%$ CL at LO (NLO).

\subsection{WIMP pair production}

Systematic uncertainties on WIMP pair production are treated similarly to those of the ADD limits, except for the PDF and ISR/FSR uncertainties. The former are determined using CTEQ6M error sets for the relative uncertainty around the CTEQ6L1 central value. The ISR/FSR uncertainties are estimated differently in a way that is appropriate for the high$p_{\mathrm{T}}$ ISR/FSR regime probed here: a WIMP pair recoils against a high- $p_{\mathrm{T}}$ ISR/FSR jet, whereas for ADD, additional low- $p_{\mathrm{T}}$ ISR/FSR jets dominate the uncertainty due to the impact of the jet veto.

The JES/JER/ $E_{\mathrm{T}}^{\text {miss }}$ experimental uncertainties lead to 1-20\% uncertainties on the WIMP event yield depending on the signal region and the effective operator considered. Other experimental uncertainties affecting the WIMP event yield are associated with the trigger efficiency (1\%) and the luminosity measurement (3.9\%). The ISR/FSR uncertainties are estimated by varying the jet matching scale between MADGRAPH5 and PYTHIA by a factor of one half and two. Moreover, the $\alpha_{s}$ scale is varied in PYTHIA within a range that is consistent with experimental data [69]. The resulting uncertainties on $\sigma \times A$, added in quadrature, range from 3-5\% for the matching scale and $4-6 \%$ for $\alpha_{s}$ depending on the signal region. A negligible dependence of the ISR/FSR uncertainties on the choice of effective operator is found. PDF uncertainties impact mostly the signal cross section and hardly the acceptance. They are found to depend on the effective operator chosen and not the particular signal region (since overall cross-section differences affect the signal regions in the same way). Uncertainties ranging from $4 \%$ and $5 \%$ for operators D9 and D5 to $16 \%$ and $18 \%$ for D11 and D1 are found. As for the ADD model, the dominating theoretical systematic uncertainty is from the factorisation and renormalisation scales. Varying these scales between twice and half their default value results in $30 \%$ signal uncertainties, independent of the effective operator choice or the signal region.

\footnotetext{
${ }^{6}$ The previous ATLAS monojet search [16] has determined ADD parameter limits in a slightly different way. The effect of the signal cross section theoretical uncertainty was folded into the quoted limit and was not shown separately.
} 

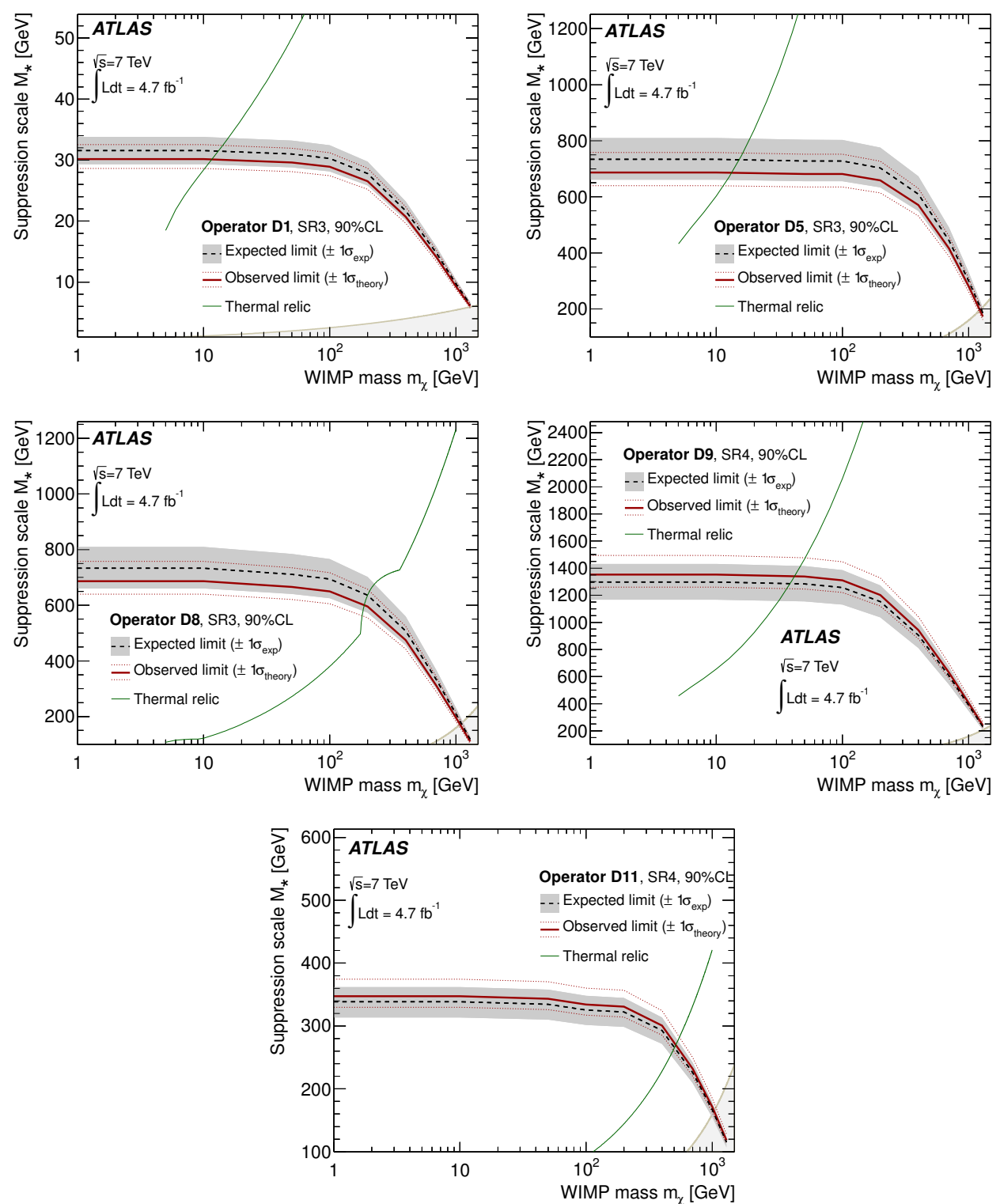

Figure 4. ATLAS lower limits at 90\% CL on $M_{*}$ for different masses of $\chi$ - the region below the limit lines is excluded. The $90 \%$ instead of the $95 \%$ CL lower limits are plotted because the former are used in the following figures 5 and 6 . Observed and expected limits including all but the theoretical signal uncertainties are shown as dashed black and red solid lines, respectively. The grey $\pm 1 \sigma$ band around the expected limit is the variation expected from statistical fluctuations and experimental systematic uncertainties on SM and signal processes. The impact of the theoretical uncertainties is shown by the thin red dotted $\pm 1 \sigma$ limit lines around the observed limit. The $M_{*}$ values at which WIMPs of a given mass would result in the required relic abundance are shown as rising green lines (taken from [32]), assuming annihilation in the early universe proceeded exclusively via the given operator. The shaded light-grey regions in the bottom right corners indicate where the effective field theory approach breaks down [32]. The plots for D1, D5, D8 are based on SR3, those for D9 and D11 on SR4. 


\begin{tabular}{|r|ccccc|}
\hline$m_{\chi}$ & D1 & D5 & D8 & D9 & D11 \\
\hline 1 & $30(29)$ & $687(658)$ & $687(658)$ & $1353(1284)$ & $347(335)$ \\
5 & $30(29)$ & $687(658)$ & $687(658)$ & $1353(1284)$ & $347(335)$ \\
10 & $30(29)$ & $687(658)$ & $687(658)$ & $1353(1284)$ & $347(335)$ \\
50 & $30(29)$ & $682(653)$ & $666(638)$ & $1338(1269)$ & $343(331)$ \\
100 & $29(28)$ & $681(653)$ & $650(623)$ & $1310(1243)$ & $334(322)$ \\
200 & $27(26)$ & $658(631)$ & $595(570)$ & $1202(1140)$ & $331(319)$ \\
400 & $21(20)$ & $571(547)$ & $475(455)$ & $943(893)$ & $301(290)$ \\
700 & $14(14)$ & $416(398)$ & $311(298)$ & $629(596)$ & $232(223)$ \\
1000 & $9(9)$ & $281(269)$ & $196(188)$ & $406(384)$ & $171(165)$ \\
1300 & $6(6)$ & $173(165)$ & $110(106)$ & $240(227)$ & $118(114)$ \\
\hline
\end{tabular}

Table 9. ATLAS 90\% (95\%) CL observed lower limits on the suppression scale $M_{*}$ as a function of WIMP mass $m_{\chi}$. All values are given in $\mathrm{GeV}$ and correspond to the nominal observed limit excluding theoretical uncertainties. The signal regions with the best expected limits are quoted in all cases, SR3 is used for D1, D5 and D8, SR4 for D9 and D11.

Figure 4 shows the $90 \%$ CL lower limits on the suppression scale $M_{*}$, for all operators probed as a function of WIMP mass $m_{\chi}$. These limits on $M_{*}$ are derived from the crosssection limits at a given mass $m_{\chi}$. The values displayed are for the signal regions with the best expected limits, where those limits from SR3 and SR4 are typically within a few percent of each other, and those from SR2 (SR1) are 15-20\% (40-50\%) smaller than in SR3 or SR4. The lower limits are based on simulation samples produced for $m_{\chi}$ between 10 and $1300 \mathrm{GeV}$. Extrapolations are shown down to $m_{\chi}=1 \mathrm{GeV}$. These are valid (and could be continued as constants to even smaller $m_{\chi}$ values entering the warm or hot dark-matter regime) since there is negligible change in cross section or kinematic distributions at the LHC for low-mass WIMPs. As before, the central values of observed and expected limits on $M_{*}$ are displayed taking into account experimental but not theoretical uncertainties. The effect of $\pm 1 \sigma$ variations on the expected limit due to statistical fluctuations and experimental uncertainties is shown as a grey band. The impact of the theoretical uncertainties is represented by dotted red $\pm 1 \sigma$ lines on either side of the observed limit. The nominal observed limit line excluding theoretical uncertainties is the final result. All values of the lower limits on the suppression scale $M_{*}$ at $90 \%$ and $95 \% \mathrm{CL}$ are listed in table 9 . For all operators, the lower limits are flat up to $m_{\chi}=100 \mathrm{GeV}$ and worsen around $m_{\chi}=200 \mathrm{GeV}$. Note that the $M_{*}$ limits for D1 are much smaller due to the inclusion of a factor $m_{q} / M_{*}$ in the definition of the operator (see table 1).

The light-grey shaded regions in figure 4 indicate where the effective field theory approach for WIMP pair production breaks down [32] (bottom-right corner in all plots). ${ }^{7}$ Except for some of the $m_{\chi}=1300 \mathrm{GeV}$ points, the $M_{*}$ limits set in this analysis are well

\footnotetext{
${ }^{7}$ Compared to ref. [32] the valid region of D1 shown here accounts for the factor of $m_{q}$ in the definition of D1 (see table 1).
} 


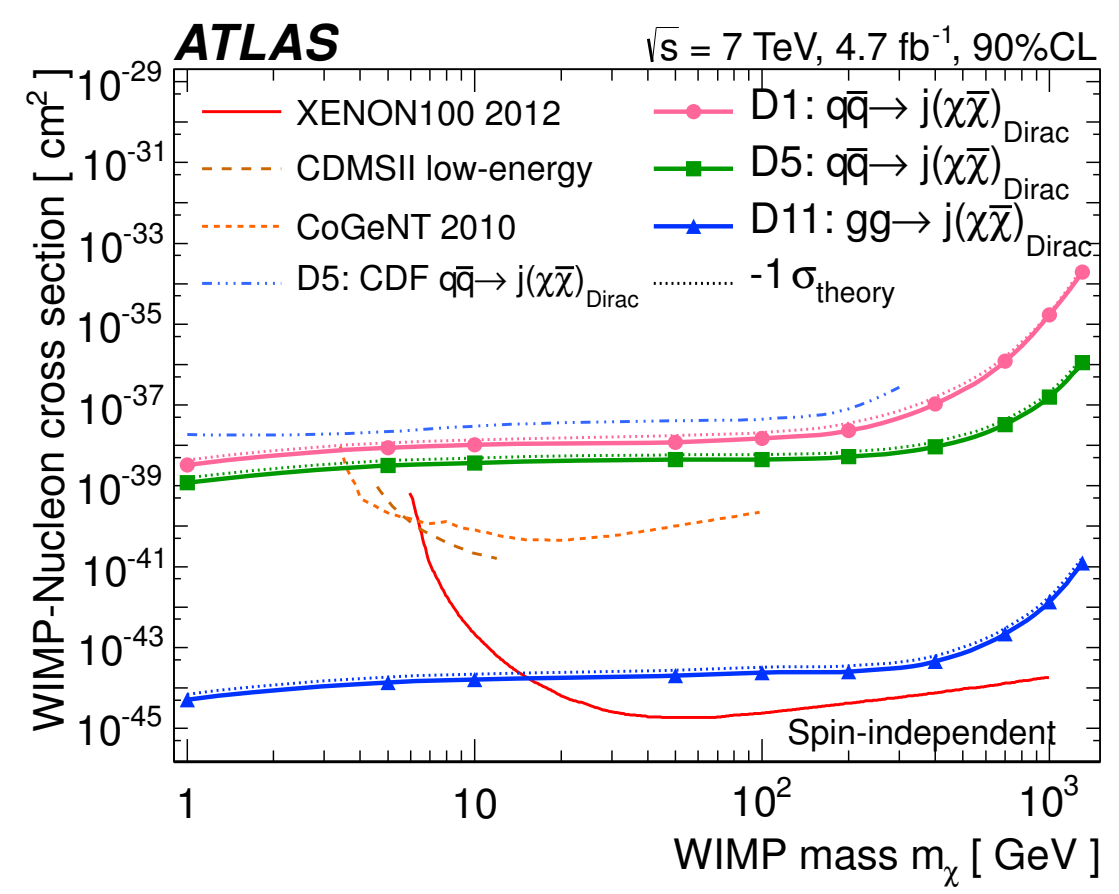

Figure 5. Inferred 90\% CL ATLAS limits on spin-independent WIMP-nucleon scattering. Cross sections are shown versus WIMP mass $m_{\chi}$. In all cases the thick solid lines are the observed limits excluding theoretical uncertainties; the observed limits corresponding to the WIMP-parton cross section obtained from the $-1 \sigma_{\text {theory }}$ lines in figure 4 are shown as thin dotted lines. The latter limits are conservative because they also include theoretical uncertainties. The ATLAS limits for operators involving quarks are for the four light flavours assuming equal coupling strengths for all quark flavours to the WIMPs. For comparison, 90\% CL limits from the XENON100 [70], CDMSII [71], CoGeNT [72], CDF [19], and CMS [21] experiments are shown.

above these bounds. No further measures are taken to ensure that the energy transfer in monojet events in this dataset remains in the valid region of the effective field theory. Such a region of validity cannot be defined without precise knowledge of the BSM physics, over which the effective operators integrate.

Figure 4 also includes thermal relic lines (taken from [32]) which correspond to a coupling, set by $M_{*}$, of WIMPs to quarks or gluons such that WIMPs have the correct relic abundance as measured by the WMAP satellite [30], in the absence of any other interaction than the one considered. Under the assumption that DM is entirely composed of thermal relics, ATLAS limits on $M_{*}$ that are above the value required for the thermal relic density exclude the case where DM annihilates exclusively to SM particles via the corresponding operator. Should thermal relic WIMPs exist in these regions (above the thermal relic line), there would have to be other annihilation channels or annihilation via other operators in order to be consistent with the WMAP measurements.

In the effective operator approach, the ATLAS bounds on $M_{*}$ for a given $m_{\chi}$ can be converted to bounds on WIMP-nucleon scattering cross sections, which are probed by direct dark matter detection experiments. These bounds describe scattering of WIMPs from nucleons at a very low momentum transfer of the order of a keV. Depending on the 


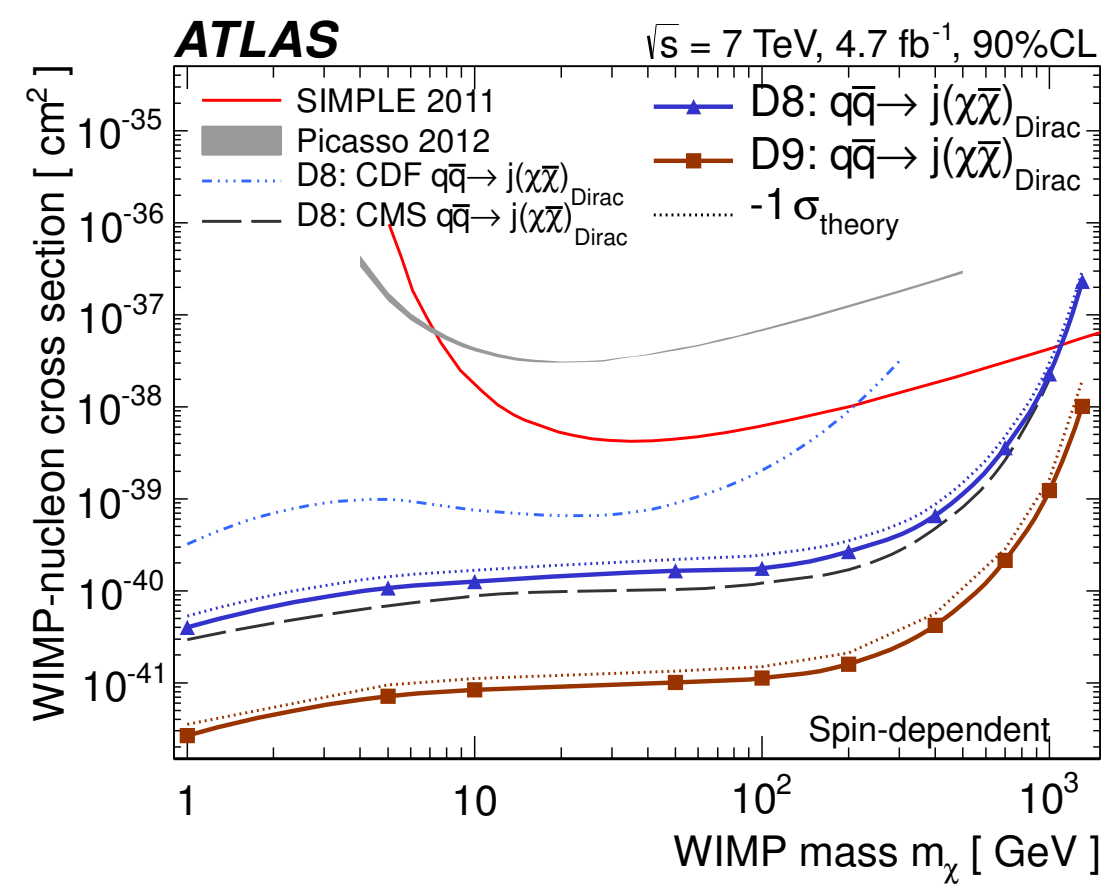

Figure 6. Inferred 90\% CL ATLAS limits on spin-dependent WIMP-nucleon scattering. Cross sections are shown versus WIMP mass $m_{\chi}$. In all cases the thick solid lines are the observed limits excluding theoretical uncertainties, the observed limits corresponding to the WIMP-parton cross section obtained from the $-1 \sigma_{\text {theory }}$ lines in figure 4 are shown as thin dotted lines. The latter limits are conservative because they also include theoretical uncertainties. The ATLAS limits are for the four light flavours assuming equal coupling strengths for all quark flavours to the WIMPs. For comparison, 90\% CL limits from the SIMPLE [73], Picasso [74], CDF [19], and CMS [21] experiments are shown.

type of interaction, contributions to spin-dependent or spin-independent WIMP-nucleon interactions are expected. The translation of ATLAS limits to bounds on WIMP-nucleon scattering cross sections is done using equations (3) to (6) of ref. [32], and the results are shown in figures 5 and $6 .^{8}$ As in ref. [32] uncertainties on hadronic matrix elements are neglected here. The spin-independent ATLAS limits in figure 5 are particularly relevant in the low $m_{\chi}$ region $(<10 \mathrm{GeV})$ where the XENON100 [70], CDMSII [71] or CoGeNT [72] limits suffer from a kinematic suppression. Should DM particles couple exclusively to gluons via D11, the collider limits would be competitive up to $m_{\chi}$ of about $20 \mathrm{GeV}$, and remain important over almost the full $m_{\chi}$ range covered. The spin-dependent limits in figure 6 are based on D8 and D9, where for D8 the $M_{*}$ limits are calculated using the D5 acceptances (as they are identical) together with D8 production cross sections. Both the D8 and D9 cross-section limits are significantly smaller than those from direct-detection experiments.

As in figure 4, the collider limits can be interpreted in terms of the relic abundance of WIMPs $[13,15]$. This is shown in figure 7 where the limits on vector and axial-vector interactions are translated into upper limits on the annihilation rate of WIMPs to the four

\footnotetext{
${ }^{8}$ There is a typographical error in equation (5) of ref. [32] (cross sections for D8 and D9). Instead of $9.18 \times 10^{-40} \mathrm{~cm}^{2}$ the pre-factor should be $4.7 \times 10^{-39} \mathrm{~cm}^{2}$.
} 
light quark flavours. The annihilation rate is defined as the product of cross section $\sigma$ and relative velocity $v$, averaged over the dark matter velocity distribution $(\langle\sigma v\rangle)$. Equations (10) and (11) of ref. [15] are used to calculate the annihilation rates shown in figure 7. For comparison, limits on annihilation to $b \bar{b}$ from Galactic high-energy gamma-ray observations by the Fermi-LAT experiment [75] are also shown. The Fermi-LAT values are for Majorana fermions and are therefore scaled up by a factor of two for comparison with the ATLAS limits for Dirac fermions (see for example the description of equation (34) of ref. [76] for an explanation of the factor of two). Gamma-ray spectra and yields from WIMPs annihilating to $b \bar{b}$, where photons are produced in the hadronisation of the quarks, are expected to be very similar to those from WIMPs annihilating to lighter quarks [77, 78]. In this sense the ATLAS and Fermi-LAT limits can be compared to each other. The figure also demonstrates the complementarity between the two approaches. The Fermi-LAT experiment is equally sensitive to annihilation to light and heavy quarks, whereas ATLAS probes mostly WIMP couplings to lighter quarks and sets cross-section limits that are superior at WIMP masses below $10 \mathrm{GeV}$ for vector couplings and below about $100 \mathrm{GeV}$ for axial-vector couplings. At these low WIMP masses, the ATLAS limits are below the value needed for WIMPs to make up the cold dark matter abundance (labelled Thermal relic value in figure 7), assuming WIMPs have annihilated exclusively via the particular operator to SM quarks while they were in thermal equilibrium in the early universe. In this case WIMPs would result in relic densities that are too large and hence incompatible with the WMAP measurements. For masses of $m_{\chi} \geq 200 \mathrm{GeV}$ the ATLAS sensitivity worsens substantially compared to the Fermi-LAT one. This will improve when the LHC starts operation at higher centre-of-mass energies in the future.

The value of using an effective field theory approach to WIMP-SM particle coupling is that only two parameters, $M_{*}$ and $m_{\chi}$, are needed to describe WIMP pair production at the LHC, WIMP-nucleon scattering measured by direct-detection experiments, and WIMP annihilation measured by indirect-detection experiments. The complementarity between the different experimental approaches can hence be explored under a number of important assumptions: the effective field theory must be valid, WIMPs must interact with SM quarks or gluons exclusively via only one of the operators of the effective field theory (since a mix of operators with potential interference effects is not considered here), and the interactions must be flavour-universal for the four light quarks. In the future, should there be a WIMP signal in at least one of the experiments from these various fields, the effective-operator approach would allow important tests of the underlying physics by probing all the available experimental data.

\section{Summary}

A search for physics beyond the Standard Model is presented in events with a high-energy jet and missing transverse momentum. The search uses the full $2011 \mathrm{pp}$ LHC dataset recorded with the ATLAS detector at a centre-of-mass energy of $\sqrt{s}=7 \mathrm{TeV}$. The data correspond to an integrated luminosity of $4.7 \mathrm{fb}^{-1}$. 


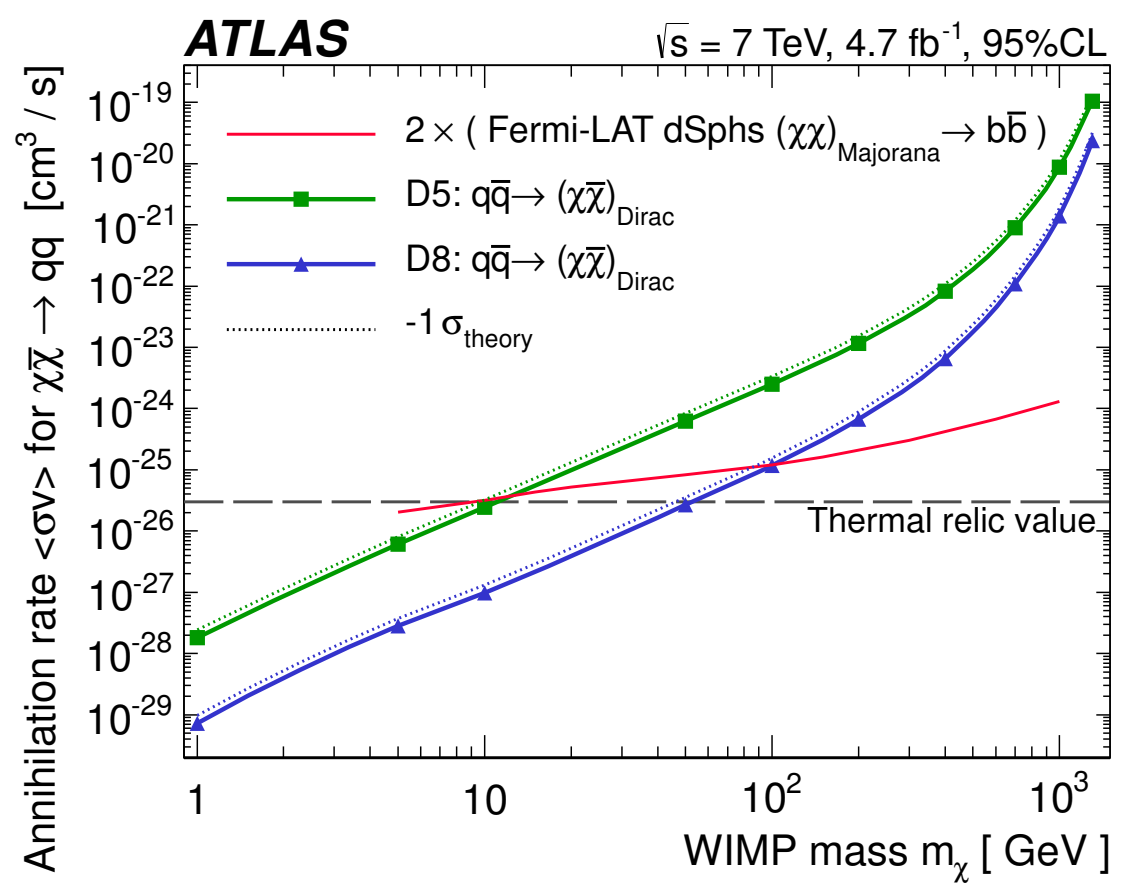

Figure 7. Inferred ATLAS 95\% CL limits on WIMP annihilation rates $\langle\sigma v\rangle$ versus mass $m_{\chi}$. $\langle\sigma v\rangle$ is calculated as in ref. [15]. The thick solid lines are the observed limits excluding theoretical uncertainties. The observed limits corresponding to the WIMP-parton cross section obtained from the $-1 \sigma_{\text {theory }}$ lines in figure 4 are shown as thin dotted lines. The latter limits are conservative because they also include theoretical uncertainties. The ATLAS limits are for the four light quark flavours assuming equal coupling strengths for all quark flavours to the WIMPs. For comparison, high-energy gamma-ray limits from observations of Galactic satellite galaxies with the Fermi-LAT experiment [75] for Majorana WIMPs are shown. The Fermi-LAT limits are scaled up by a factor of two to make them comparable to the ATLAS Dirac WIMP limits. All limits shown here assume $100 \%$ branching fractions of WIMPs annihilating to quarks. The horizontal dashed line indicates the value required for WIMPs to make up the relic abundance set by the WMAP measurement.

Four overlapping signal regions are defined for the search. They require a high-energy jet and missing transverse momentum of at least $120,220,350$ and $500 \mathrm{GeV}$, with at most one additional jet not aligned with the direction of $E_{\mathrm{T}}^{\text {miss }}$ (to suppress multijet background). In all cases the events are required to contain no identified electrons or muons. The dominant Standard Model backgrounds from $Z$ and $W$ plus jet production, where the boson decays to a final state that includes 1-2 neutrinos, are determined using data control regions with correction and transfer factors determined from data and simulations. This technique allows precise estimates of the SM contributions to monojet final states, which is reflected in a small total uncertainty of $3.2 \%$ for the background prediction in the highstatistics signal region SR1.

In each of the four signal regions, agreement is found between the Standard Model predictions and the data. Upper limits are set at $95 \% \mathrm{CL}$ on the visible cross section of any non-SM contribution to the signal regions. These limits range from $1.92 \mathrm{pb}$ in the first signal region to $7 \mathrm{fb}$ in the fourth signal region. To allow comparisons with the results 
of other experiments, 90\% CL limits are also provided. The cross-section upper limits are interpreted in terms of limits on the model parameters of two BSM physics scenarios. For ADD, a model of large extra spatial dimensions, lower limits are set on the $(4+n)$ dimensional Planck scale $M_{D}$ of $4.17(2.51) \mathrm{TeV}$ for $n=2(6)$ extra dimensions at LO and $4.37(2.53) \mathrm{TeV}$ at NLO. In a second scenario an effective field theory is used to derive limits on a mass suppression scale $M_{*}$ for pair production of WIMP dark matter particles. Within this approach the ATLAS limits can be converted to limits on WIMP-nucleon scattering and WIMP annihilation cross sections. Assuming the effective field theory is valid, that WIMPs interact with SM quarks or gluons, and that they can be pair-produced at the LHC, some of the limits are competitive with or substantially better than limits set by direct and indirect dark matter detection experiments, in particular at small WIMP masses of $m_{\chi}<10 \mathrm{GeV}$.

\section{Acknowledgments}

We would like to thank Tim Tait for providing the dark matter generator software and supporting us during its validation.

We thank CERN for the very successful operation of the LHC, as well as the support staff from our institutions without whom ATLAS could not be operated efficiently.

We acknowledge the support of ANPCyT, Argentina; YerPhI, Armenia; ARC, Australia; BMWF and FWF, Austria; ANAS, Azerbaijan; SSTC, Belarus; CNPq and FAPESP, Brazil; NSERC, NRC and CFI, Canada; CERN; CONICYT, Chile; CAS, MOST and NSFC, China; COLCIENCIAS, Colombia; MSMT CR, MPO CR and VSC CR, Czech Republic; DNRF, DNSRC and Lundbeck Foundation, Denmark; EPLANET and ERC, European Union; IN2P3-CNRS, CEA-DSM/IRFU, France; GNSF, Georgia; BMBF, DFG, HGF, MPG and AvH Foundation, Germany; GSRT, Greece; ISF, MINERVA, GIF, DIP and Benoziyo Center, Israel; INFN, Italy; MEXT and JSPS, Japan; CNRST, Morocco; FOM and NWO, Netherlands; BRF and RCN, Norway; MNiSW, Poland; GRICES and FCT, Portugal; MERYS (MECTS), Romania; MES of Russia and ROSATOM, Russian Federation; JINR; MSTD, Serbia; MSSR, Slovakia; ARRS and MVZT, Slovenia; DST/NRF, South Africa; MICINN, Spain; SRC and Wallenberg Foundation, Sweden; SER, SNSF and Cantons of Bern and Geneva, Switzerland; NSC, Taiwan; TAEK, Turkey; STFC, the Royal Society and Leverhulme Trust, United Kingdom; DOE and NSF, United States of America.

The crucial computing support from all WLCG partners is acknowledged gratefully, in particular from CERN and the ATLAS Tier-1 facilities at TRIUMF (Canada), NDGF (Denmark, Norway, Sweden), CC-IN2P3 (France), KIT/GridKA (Germany), INFN-CNAF (Italy), NL-T1 (Netherlands), PIC (Spain), ASGC (Taiwan), RAL (UK) and BNL (USA) and in the Tier-2 facilities worldwide.

Open Access. This article is distributed under the terms of the Creative Commons Attribution License which permits any use, distribution and reproduction in any medium, provided the original author(s) and source are credited. 


\section{References}

[1] H. Miyazawa, Baryon number changing currents, Prog. Theor. Phys. 36 (1966) 1266.

[2] P. Ramond, Dual theory for free fermions, Phys. Rev. D 3 (1971) 2415 [InSPIRE].

[3] Y. Golfand and E. Likhtman, Extension of the algebra of Poincaré group generators and violation of $p$ invariance, JETP Lett. 13 (1971) 323 [INSPIRE].

[4] A. Neveu and J. Schwarz, Factorizable dual model of pions, Nucl. Phys. B 31 (1971) 86 [INSPIRE].

[5] A. Neveu and J. Schwarz, Quark model of dual pions, Phys. Rev. D 4 (1971) 1109 [INSPIRE].

[6] J.-L. Gervais and B. Sakita, Field theory interpretation of supergauges in dual models, Nucl. Phys. B 34 (1971) 632 [inSPIRE].

[7] D. Volkov and V. Akulov, Is the neutrino a Goldstone particle?, Phys. Lett. B 46 (1973) 109 [INSPIRE].

[8] J. Wess and B. Zumino, A lagrangian model invariant under supergauge transformations, Phys. Lett. B 49 (1974) 52 [INSPIRE].

[9] J. Wess and B. Zumino, Supergauge transformations in four-dimensions, Nucl. Phys. B 70 (1974) 39 [INSPIRE].

[10] M. Carena, A. Freitas and C. Wagner, Light stop searches at the LHC in events with one hard photon or jet and missing energy, JHEP 10 (2008) 109 [arXiv:0808.2298] [INSPIRE].

[11] B.C. Allanach, S. Grab and H.E. Haber, Supersymmetric monojets at the Large Hadron Collider, JHEP 01 (2011) 138 [Erratum ibid. 1107 (2011) 087] [arXiv:1010.4261] [INSPIRE].

[12] N. Arkani-Hamed, S. Dimopoulos and G. Dvali, The hierarchy problem and new dimensions at a millimeter, Phys. Lett. B 429 (1998) 263 [hep-ph/9803315] [InSPIRE].

[13] M. Beltrán, D. Hooper, E.W. Kolb, Z.A. Krusberg and T.M. Tait, Maverick dark matter at colliders, JHEP 09 (2010) 037 [arXiv: 1002.4137] [INSPIRE].

[14] A. Rajaraman, W. Shepherd, T.M. Tait and A.M. Wijangco, LHC bounds on interactions of dark matter, Phys. Rev. D 84 (2011) 095013 [arXiv:1108.1196] [InSPIRE].

[15] P.J. Fox, R. Harnik, J. Kopp and Y. Tsai, Missing energy signatures of dark matter at the LHC, Phys. Rev. D 85 (2012) 056011 [arXiv:1109.4398] [InSPIRE].

[16] ATLAS collaboration, Search for new phenomena with the monojet and missing transverse momentum signature using the ATLAS detector in $\sqrt{s}=7$ TeV proton-proton collisions, Phys. Lett. B 705 (2011) 294 [arXiv:1106.5327] [INSPIRE].

[17] D0 collaboration, V. Abazov et al., Search for large extra dimensions in the monojet + missing $E_{T}$ channel at DØ, Phys. Rev. Lett. 90 (2003) 251802 [hep-ex/0302014] [INSPIRE].

[18] CDF collaboration, A. Abulencia et al., Search for large extra dimensions in the production of jets and missing transverse energy in p $\bar{p}$ collisions at $\sqrt{s}=1.96 \mathrm{TeV}$, Phys. Rev. Lett. 97 (2006) 171802 [hep-ex/0605101] [INSPIRE].

[19] CDF collaboration, T. Aaltonen et al., A search for dark matter in events with one jet and missing transverse energy in $p \bar{p}$ collisions at $\sqrt{s}=1.96 \mathrm{TeV}$, Phys. Rev. Lett. 108 (2012) 211804 [arXiv:1203.0742] [INSPIRE]. 
[20] CMS collaboration, Search for new physics with a mono-jet and missing transverse energy in pp collisions at $\sqrt{s}=7$ TeV, Phys. Rev. Lett. 107 (2011) 201804 [arXiv:1106.4775] [INSPIRE].

[21] CMS collaboration, Search for dark matter and large extra dimensions in monojet events in pp collisions at $\sqrt{s}=7$ TeV, JHEP 09 (2012) 094 [arXiv:1206.5663] [INSPIRE].

[22] S. Weinberg, Implications of dynamical symmetry breaking, Phys. Rev. D 13 (1976) 974 [INSPIRE].

[23] E. Gildener, Gauge symmetry hierarchies, Phys. Rev. D 14 (1976) 1667 [INSPIRE].

[24] S. Weinberg, Implications of dynamical symmetry breaking: an addendum, Phys. Rev. D 19 (1979) 1277 [INSPIRE].

[25] L. Susskind, Dynamics of spontaneous symmetry breaking in the Weinberg-Salam theory, Phys. Rev. D 20 (1979) 2619 [INSPIRE].

[26] G.F. Giudice, R. Rattazzi and J.D. Wells, Quantum gravity and extra dimensions at high-energy colliders, Nucl. Phys. B 544 (1999) 3 [hep-ph/9811291] [InSPIRE].

[27] G. Bertone, D. Hooper and J. Silk, Particle dark matter: evidence, candidates and constraints, Phys. Rept. 405 (2005) 279 [hep-ph/0404175] [INSPIRE].

[28] G. Steigman and M.S. Turner, Cosmological constraints on the properties of weakly interacting massive particles, Nucl. Phys. B 253 (1985) 375 [InSPIRE].

[29] E.W. Kolb and M.S. Turner, The early universe, Front. Phys. 69 (1990) 1 [INSPIRE].

[30] WMAP collaboration, E. Komatsu et al., Seven-year Wilkinson Microwave Anisotropy Probe (WMAP) observations: cosmological interpretation, Astrophys. J. Suppl. 192 (2011) 18 [arXiv:1001.4538] [INSPIRE].

[31] A. Birkedal, K. Matchev and M. Perelstein, Dark matter at colliders: a model independent approach, Phys. Rev. D 70 (2004) 077701 [hep-ph/0403004] [INSPIRE].

[32] J. Goodman et al., Constraints on dark matter from colliders, Phys. Rev. D 82 (2010) 116010 [arXiv: 1008.1783] [InSPIRE].

[33] A. Friedland, M.L. Graesser, I.M. Shoemaker and L. Vecchi, Probing nonstandard standard model backgrounds with LHC monojets, Phys. Lett. B 714 (2012) 267 [arXiv:1111.5331] [INSPIRE].

[34] ATLAS collaboration, The ATLAS experiment at the CERN Large Hadron Collider, 2008 JINST 3 S08003 [INSPIRE].

[35] ATLAS collaboration, Expected performance of the ATLAS experiment - Detector, trigger and physics, arXiv:0901.0512 [INSPIRE].

[36] ATLAS collaboration, Luminosity determination in pp collisions at $\sqrt{s}=7$ TeV using the ATLAS detector at the LHC, Eur. Phys. J. C 71 (2011) 1630 [arXiv:1101.2185] [INSPIRE].

[37] ATLAS collaboration, Luminosity determination in pp collisions at $\sqrt{s}=7$ TeV using the ATLAS detector in 2011, ATLAS-CONF-2011-116 (2011).

[38] M.L. Mangano, M. Moretti, F. Piccinini, R. Pittau and A.D. Polosa, ALPGEN, a generator for hard multiparton processes in hadronic collisions, JHEP 07 (2003) 001 [hep-ph/0206293] [INSPIRE]. 
[39] J. Pumplin et al., New generation of parton distributions with uncertainties from global QCD analysis, JHEP 07 (2002) 012 [hep-ph/0201195] [INSPIRE].

[40] G. Corcella et al., HERWIG 6: an event generator for hadron emission reactions with interfering gluons (including supersymmetric processes), JHEP 01 (2001) 010 [hep-ph/0011363] [INSPIRE].

[41] G. Corcella et al., HERWIG 6.5 release note, hep-ph/0210213 [INSPIRE].

[42] M.L. Mangano, M. Moretti, F. Piccinini and M. Treccani, Matching matrix elements and shower evolution for top-quark production in hadronic collisions, JHEP 01 (2007) 013 [hep-ph/0611129] [INSPIRE].

[43] J. Butterworth, J.R. Forshaw and M. Seymour, Multiparton interactions in photoproduction at HERA, Z. Phys. C 72 (1996) 637 [hep-ph/9601371] [INSPIRE].

[44] T. Gleisberg et al., Event generation with SHERPA 1.1, JHEP 02 (2009) 007 [arXiv: 0811.4622] [INSPIRE].

[45] S. Frixione and B.R. Webber, The MC@NLO 3.2 event generator, hep-ph/0601192 [INSPIRE].

[46] P.M. Nadolsky et al., Implications of CTEQ global analysis for collider observables, Phys. Rev. D 78 (2008) 013004 [arXiv:0802.0007] [INSPIRE].

[47] T. Sjöstrand, S. Mrenna and P.Z. Skands, PYTHIA 6.4 physics and manual, JHEP 05 (2006) 026 [hep-ph/0603175] [INSPIRE].

[48] A. Martin, W. Stirling, R. Thorne and G. Watt, Parton distributions for the LHC, Eur. Phys. J. C 63 (2009) 189 [arXiv:0901.0002] [InSPIRE].

[49] S. Karg, M. Krämer, Q. Li and D. Zeppenfeld, NLO QCD corrections to graviton production at hadron colliders, Phys. Rev. D 81 (2010) 094036 [arXiv:0911.5095] [INSPIRE].

[50] J. Alwall, M. Herquet, F. Maltoni, O. Mattelaer and T. Stelzer, MadGraph 5: going beyond, JHEP 06 (2011) 128 [arXiv:1106.0522] [INSPIRE].

[51] ATLAS collaboration, The ATLAS simulation infrastructure, Eur. Phys. J. C 70 (2010) 823 [arXiv: 1005.4568] [inSPIRE].

[52] GEANT4 collaboration, S. Agostinelli et al., GEANT4: a simulation toolkit, Nucl. Instrum. Meth. A 506 (2003) 250 [inSPIRE].

[53] M. Cacciari, G.P. Salam and G. Soyez, The anti- $k_{t}$ jet clustering algorithm, JHEP 04 (2008) 063 [arXiv: 0802.1189] [INSPIRE].

[54] ATLAS collaboration, Calorimeter clustering algorithms: description and performance, ATL-LARG-PUB-2008-002 (2008).

[55] ATLAS collaboration, Jet energy measurement with the ATLAS detector in proton-proton collisions at $\sqrt{s}=7 \mathrm{TeV}$, Eur. Phys. J. C 73 (2013) 2304 [arXiv:1112.6426] [InSPIRE].

[56] ATLAS collaboration, Electron performance measurements with the ATLAS detector using the 2010 LHC proton-proton collision data, Eur. Phys. J. C 72 (2012) 1909 [arXiv:1110.3174] [INSPIRE].

[57] ATLAS collaboration, Measurement of the $W \rightarrow \ell \nu$ and $Z / \gamma^{*} \rightarrow \ell \ell$ production cross sections in proton-proton collisions at $\sqrt{s}=7 \mathrm{TeV}$ with the ATLAS detector, JHEP 12 (2010) 060 [arXiv:1010.2130] [InSPIRE]. 
[58] ATLAS collaboration, Local hadron calibration, ATL-LARG-PUB-2009-001 (2009).

[59] ATLAS collaboration, Performance of missing transverse momentum reconstruction in proton-proton collisions at $7 \mathrm{TeV}$ with ATLAS, Eur. Phys. J. C 72 (2012) 1844 [arXiv:1108.5602] [INSPIRE].

[60] ATLAS collaboration, The implementation of the ATLAS missing $E_{\mathrm{t}}$ triggers for the initial LHC operation, ATL-DAQ-PUB-2011-001 (2011).

[61] ATLAS collaboration, Performance of the ATLAS transverse energy triggers with initial LHC runs at $\sqrt{s}=7 \mathrm{TeV}$, ATLAS-CONF-2011-072 (2011).

[62] ATLAS collaboration, Performance of the ATLAS inner detector track and vertex reconstruction in the high pile-up LHC environment, ATLAS-CONF-2012-042 (2012).

[63] ATLAS collaboration, Data-quality requirements and event cleaning for jets and missing transverse energy reconstruction with the ATLAS detector in proton-proton collisions at a center-of-mass energy of $\sqrt{s}=7 \mathrm{TeV}$, ATLAS-CONF-2010-038 (2010).

[64] ATLAS collaboration, Non-collision backgrounds as measured by the ATLAS detector during the 2010 proton-proton run, ATLAS-CONF-2011-137 (2011).

[65] ATLAS collaboration, Measurement of the inclusive $W^{ \pm}$and $Z / \gamma$ cross sections in the electron and muon decay channels in pp collisions at $\sqrt{s}=7 \mathrm{TeV}$ with the ATLAS detector, Phys. Rev. D 85 (2012) 072004 [arXiv:1109.5141] [INSPIRE].

[66] ATLAS collaboration, $A$ measurement of the ratio of the $W$ and $Z$ cross sections with exactly one associated jet in pp collisions at $\sqrt{s}=7 \mathrm{TeV}$ with ATLAS, Phys. Lett. B 708 (2012) 221 [arXiv:1108.4908] [INSPIRE].

[67] A.L. Read, Presentation of search results: the $C L_{s}$ technique, J. Phys. G 28 (2002) 2693 [INSPIRE].

[68] G. Cowan, K. Cranmer, E. Gross and O. Vitells, Asymptotic formulae for likelihood-based tests of new physics, Eur. Phys. J. C 71 (2011) 1554 [arXiv:1007.1727] [INSPIRE].

[69] P.Z. Skands, Tuning Monte Carlo generators: the Perugia tunes, Phys. Rev. D 82 (2010) 074018 [arXiv: 1005.3457] [InSPIRE].

[70] XENON100 collaboration, E. Aprile et al., Dark matter results from 225 live days of XENON100 data, Phys. Rev. Lett. 109 (2012) 181301 [arXiv:1207.5988] [InSPIRE].

[71] CDMS-II collaboration, Z. Ahmed et al., Results from a low-energy analysis of the CDMS II Germanium data, Phys. Rev. Lett. 106 (2011) 131302 [arXiv:1011.2482] [INSPIRE].

[72] CoGeNT collaboration, C. Aalseth et al., Results from a search for light-mass dark matter with a p-type point contact Germanium detector, Phys. Rev. Lett. 106 (2011) 131301 [arXiv: 1002.4703] [INSPIRE].

[73] M. Felizardo et al., Final analysis and results of the phase II SIMPLE dark matter search, Phys. Rev. Lett. 108 (2012) 201302 [arXiv:1106.3014] [INSPIRE].

[74] PICASSO collaboration, S. Archambault et al., Constraints on low-mass WIMP interactions on ${ }^{19}$ F from PICASSO, Phys. Lett. B 711 (2012) 153 [arXiv:1202.1240] [INSPIRE].

[75] Fermi-LAT collaboration, M. Ackermann et al., Constraining dark matter models from a combined analysis of Milky Way satellites with the Fermi Large Area Telescope, Phys. Rev. Lett. 107 (2011) 241302 [arXiv:1108.3546] [INSPIRE]. 
[76] M. Cirelli et al., PPPC \& DM ID: a poor particle physicist cookbook for dark matter indirect detection, JCAP 03 (2011) 051 [Erratum ibid. 1210 (2012) E01] [arXiv:1012.4515] [INSPIRE].

[77] L. Bergstrom, P. Ullio and J.H. Buckley, Observability of gamma-rays from dark matter neutralino annihilations in the Milky Way halo, Astropart. Phys. 9 (1998) 137 [astro-ph/9712318] [INSPIRE].

[78] N. Fornengo, L. Pieri and S. Scopel, Neutralino annihilation into $\gamma$-rays in the Milky Way and in external galaxies, Phys. Rev. D 70 (2004) 103529 [hep-ph/0407342] [INSPIRE]. 


\section{The ATLAS collaboration}

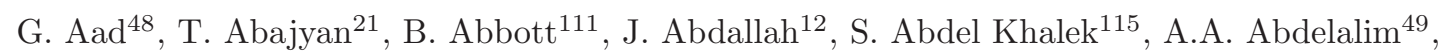
O. Abdinov ${ }^{11}$, R. Aben ${ }^{105}$, B. Abi ${ }^{112}$, M. Abolins ${ }^{88}$, O.S. AbouZeid ${ }^{158}$, H. Abramowicz ${ }^{153}$, H. Abreu ${ }^{136}$, B.S. Acharya ${ }^{164 a, 164 b}$, L. Adamczyk ${ }^{38}$, D.L. Adams ${ }^{25}$, T.N. Addy ${ }^{56}$, J. Adelman ${ }^{176}$, S. Adomeit ${ }^{98}$, P. Adragna ${ }^{75}$, T. Adye ${ }^{129}$, S. Aefsky ${ }^{23}$, J.A. Aguilar-Saavedra ${ }^{124 b, a}$, M. Agustoni ${ }^{17}$, M. Aharrouche ${ }^{81}$, S.P. Ahlen ${ }^{22}$, F. Ahles ${ }^{48}$, A. Ahmad ${ }^{148}$, M. Ahsan ${ }^{41}$, G. Aielli ${ }^{133 a, 133 b}$, T.P.A. Åkesson ${ }^{79}$, G. Akimoto ${ }^{155}$, A.V. Akimov ${ }^{94}$, M.S. Alam², M.A. Alam ${ }^{76}$, J. Albert ${ }^{169}$,

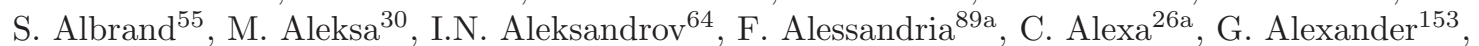
G. Alexandre ${ }^{49}$, T. Alexopoulos ${ }^{10}$, M. Alhroob ${ }^{164 a, 164 c}$, M. Aliev ${ }^{16}$, G. Alimonti ${ }^{89 a}$, J. Alison ${ }^{120}$, B.M.M. Allbrooke ${ }^{18}$, P.P. Allport ${ }^{73}$, S.E. Allwood-Spiers ${ }^{53}$, J. Almond ${ }^{82}$, A. Aloisio ${ }^{102 a, 102 b}$, R. Alon ${ }^{172}$, A. Alonso ${ }^{79}$, F. Alonso ${ }^{70}$, A. Altheimer ${ }^{35}$, B. Alvarez Gonzalez ${ }^{88}$,

M.G. Alviggi ${ }^{102 a, 102 b}$, K. Amako ${ }^{65}$, C. Amelung ${ }^{23}$, V.V. Ammosov ${ }^{128, *}$, S.P. Amor Dos Santos ${ }^{124 a}$, A. Amorim ${ }^{124 a, b}$, N. Amram ${ }^{153}$, C. Anastopoulos ${ }^{30}$, L.S. Ancu ${ }^{17}$, N. Andari ${ }^{115}$, T. Andeen ${ }^{35}$, C.F. Anders ${ }^{58 b}$, G. Anders ${ }^{58 a}$, K.J. Anderson ${ }^{31}$, A. Andreazza ${ }^{89 a, 89 b}$, V. Andrei ${ }^{58 a}$, M-L. Andrieux ${ }^{55}$, X.S. Anduaga ${ }^{70}$, S. Angelidakis ${ }^{9}$, P. Anger $^{44}$, A. Angerami ${ }^{35}$, F. Anghinolfi ${ }^{30}$, A. Anisenkov ${ }^{107}$, N. Anjos ${ }^{124 a}$, A. Annovi ${ }^{47}$, A. Antonaki ${ }^{9}$, M. Antonelli ${ }^{47}$, A. Antonov ${ }^{96}$, J. Antos ${ }^{144 b}$, F. Anulli ${ }^{132 a}$, M. Aoki ${ }^{101}$, S. Aoun ${ }^{83}$, L. Aperio Bella ${ }^{5}$, R. Apolle ${ }^{118, c}$, G. Arabidze ${ }^{88}$, I. Aracena ${ }^{143}$, Y. Arai ${ }^{65}$, A.T.H. Arce ${ }^{45}$, S. Arfaoui ${ }^{148}$, J-F. Arguin ${ }^{93}$, S. Argyropoulos ${ }^{42}$, E. Arik ${ }^{19 a, *}$, M. Arik ${ }^{19 a}$, A.J. Armbruster ${ }^{87}$, O. Arnaez ${ }^{81}$, V. Arnal ${ }^{80}$, C. Arnault ${ }^{115}$, A. Artamonov ${ }^{95}$, G. Artoni ${ }^{132 a, 132 b}$, D. Arutinov ${ }^{21}$, S. Asai ${ }^{155}$, S. Ask ${ }^{28}$, B. Åsman 146a,146b, L. Asquith ${ }^{6}$, K. Assamagan ${ }^{25}$, A. Astbury ${ }^{169}$, M. Atkinson ${ }^{165}$, B. Aubert ${ }^{5}$, E. Auge ${ }^{115}$, K. Augsten ${ }^{127}$, M. Aurousseau ${ }^{145 a}$, G. Avolio ${ }^{30}$, R. Avramidou ${ }^{10}$, D. Axen ${ }^{168}$, G. Azuelos ${ }^{93, d}$, Y. Azuma ${ }^{155}$, M.A. Baak ${ }^{30}$, G. Baccaglioni ${ }^{89 a}$, C. Bacci ${ }^{134 a, 134 b}$, A.M. Bach ${ }^{15}$, H. Bachacou ${ }^{136}$, K. Bachas ${ }^{30}$, M. Backes ${ }^{49}$, M. Backhaus ${ }^{21}$, J. Backus Mayes ${ }^{143}$, E. Badescu ${ }^{26 a}$, P. Bagnaia ${ }^{132 a, 132 b}$, S. Bahinipati ${ }^{3}$, Y. Bai ${ }^{33 a}$, D.C. Bailey ${ }^{158}$, T. Bain ${ }^{158}$, J.T. Baines ${ }^{129}$, O.K. Baker ${ }^{176}$, M.D. Baker ${ }^{25}$, S. Baker ${ }^{77}$, P. Balek ${ }^{126}$, E. Banas ${ }^{39}$, P. Banerjee ${ }^{93}$, Sw. Banerjee ${ }^{173}$, D. Banfic ${ }^{30}$ A. Bangert ${ }^{150}$, V. Bansal ${ }^{169}$, H.S. Bansil ${ }^{18}$, L. Barak ${ }^{172}$, S.P. Baranov ${ }^{94}$, A. Barbaro Galtieri ${ }^{15}$, T. Barber ${ }^{48}$, E.L. Barberio ${ }^{86}$, D. Barberis ${ }^{50 a, 50 b}$, M. Barbero ${ }^{21}$, D.Y. Bardin ${ }^{64}$, T. Barillari ${ }^{99}$, M. Barisonzi ${ }^{175}$, T. Barklow ${ }^{143}$, N. Barlow ${ }^{28}$, B.M. Barnett ${ }^{129}$, R.M. Barnett ${ }^{15}$, A. Baroncelli ${ }^{134 a}$, G. Barone ${ }^{49}$, A.J. Barr ${ }^{118}$, F. Barreiro ${ }^{80}$, J. Barreiro Guimarães da Costa ${ }^{57}$, P. Barrillon ${ }^{115}$, R. Bartoldus ${ }^{143}$, A.E. Barton ${ }^{71}$, V. Bartsch ${ }^{149}$, A. Basye $^{165}$, R.L. Bates ${ }^{53}$, L. Batkova ${ }^{144 a}$, J.R. Batley ${ }^{28}$, A. Battaglia ${ }^{17}$, M. Battistin ${ }^{30}$, F. Bauer ${ }^{136}$, H.S. Bawa ${ }^{143, e}$, S. Beale ${ }^{98}$, T. Beau ${ }^{78}$, P.H. Beauchemin ${ }^{161}$, R. Beccherle ${ }^{50 a}$, P. Bechtle ${ }^{21}$, H.P. Beck ${ }^{17}$, A.K. Becker ${ }^{175}$, S. Becker ${ }^{98}$, M. Beckingham ${ }^{138}$, K.H. Becks ${ }^{175}$, A.J. Beddall ${ }^{19 c}$, A. Beddall ${ }^{19 c}$, S. Bedikian ${ }^{176}$, V.A. Bednyakov ${ }^{64}$, C.P. Bee ${ }^{83}$, L.J. Beemster ${ }^{105}$, M. Begel ${ }^{25}$, S. Behar Harpaz ${ }^{152}$, P.K. Behera ${ }^{62}$, M. Beimforde ${ }^{99}$, C. Belanger-Champagne ${ }^{85}$, P.J. Bell ${ }^{49}$, W.H. Bell ${ }^{49}$, G. Bella ${ }^{153}$, L. Bellagamba ${ }^{20 a}$, M. Bellomo ${ }^{30}$, A. Belloni ${ }^{57}$, O. Beloborodova ${ }^{107, f}$, K. Belotskiy ${ }^{96}$, O. Beltramello ${ }^{30}$, O. Benary ${ }^{153}$, D. Benchekroun ${ }^{135 a}$, K. Bendtz ${ }^{146 a, 146 b}$, N. Benekos ${ }^{165}$, Y. Benhammou ${ }^{153}$, E. Benhar Noccioli ${ }^{49}$, J.A. Benitez Garcia ${ }^{159 b}$, D.P. Benjamin ${ }^{45}$, M. Benoit ${ }^{115}$, J.R. Bensinger ${ }^{23}$, K. Benslama ${ }^{130}$, S. Bentvelsen ${ }^{105}$, D. Berge ${ }^{30}$, E. Bergeaas Kuutmann ${ }^{42}$, N. Berger ${ }^{5}$, F. Berghaus ${ }^{169}$, E. Berglund ${ }^{105}$, J. Beringer ${ }^{15}$, P. Bernat ${ }^{77}$, R. Bernhard ${ }^{48}$, C. Bernius ${ }^{25}$, T. Berry ${ }^{76}$, C. Bertella ${ }^{83}$, A. Bertin ${ }^{20 a, 20 b}$, F. Bertolucci ${ }^{122 a, 122 b}$, M.I. Besana ${ }^{89 a, 89 b}$, G.J. Besjes ${ }^{104}$, N. Besson ${ }^{136}$, S. Bethke ${ }^{99}$, W. Bhimji ${ }^{46}$, R.M. Bianchi ${ }^{30}$, L. Bianchini ${ }^{23}$, M. Bianco ${ }^{72 a, 72 b}$, O. Biebel ${ }^{98}$, S.P. Bieniek ${ }^{77}$, K. Bierwagen ${ }^{54}$, J. Biesiada ${ }^{15}$, M. Biglietti ${ }^{134 a}$, H. Bilokon ${ }^{47}$, M. Bindi ${ }^{20 a, 20 b}$, S. Binet ${ }^{115}$, A. Bingul ${ }^{19 c}$, C. Bini ${ }^{132 a, 132 b}$, C. Biscarat ${ }^{178}$, B. Bittner ${ }^{99}$, K.M. Black ${ }^{22}$, R.E. Blair ${ }^{6}$, J.-B. Blanchard ${ }^{136}$, G. Blanchot ${ }^{30}$, T. Blazek ${ }^{144 a}$, I. Bloch ${ }^{42}$, C. Blocker ${ }^{23}$, J. Blocki ${ }^{39}$, A. Blondel ${ }^{49}$, W. Blum ${ }^{81}$, U. Blumenschein ${ }^{54}$, G.J. Bobbink ${ }^{105}$, V.B. Bobrovnikov ${ }^{107}$, S.S. Bocchetta ${ }^{79}$, A. Bocci ${ }^{45}$, C.R. Boddy ${ }^{118}$, M. Boehler ${ }^{48}$, J. Boek ${ }^{175}$, N. Boelaert ${ }^{36}$, J.A. Bogaerts ${ }^{30}$, A. Bogdanchikov ${ }^{107}$, A. Bogouch ${ }^{90, *}$, C. Bohm ${ }^{146 a}$, J. Bohm ${ }^{125}$, V. Boisvert ${ }^{76}$, T. Bold ${ }^{38}$, V. Boldea ${ }^{26 a}$, N.M. Bolnet ${ }^{136}$, M. Bomben $^{78}$, M. Bona ${ }^{75}$, M. Boonekamp ${ }^{136}$, S. Bordoni ${ }^{78}$, C. Borer ${ }^{17}$, A. Borisov ${ }^{128}$, G. Borissov ${ }^{71}$, I. Borjanovic ${ }^{13 a}$, 
M. Borri ${ }^{82}$, S. Borroni ${ }^{87}$, J. Bortfeldt ${ }^{98}$, V. Bortolotto ${ }^{134 a, 134 b}$, K. Bos $^{105}$, D. Boscherini ${ }^{20 a}$, M. Bosman ${ }^{12}$, H. Boterenbrood ${ }^{105}$, J. Bouchami ${ }^{93}$, J. Boudreau ${ }^{123}$, E.V. Bouhova-Thacker ${ }^{71}$, D. Boumediene ${ }^{34}$, C. Bourdarios ${ }^{115}$, N. Bousson ${ }^{83}$, A. Boveia ${ }^{31}$, J. Boyd ${ }^{30}$, I.R. Boyko ${ }^{64}$, I. Bozovic-Jelisavcic ${ }^{13 b}$, J. Bracinik ${ }^{18}$, P. Branchini ${ }^{134 a}$, A. Brandt ${ }^{8}$, G. Brandt ${ }^{118}$, O. Brandt ${ }^{54}$, U. Bratzler ${ }^{156}$, B. Brau ${ }^{84}$, J.E. Brau ${ }^{114}$, H.M. Braun ${ }^{175, *}$, S.F. Brazzale ${ }^{164 a, 164 c}$, B. Brelier ${ }^{158}$, J. Bremer ${ }^{30}$, K. Brendlinger ${ }^{120}$, R. Brenner ${ }^{166}$, S. Bressler ${ }^{172}$, D. Britton ${ }^{53}$, F.M. Brochu ${ }^{28}$, I. Brock ${ }^{21}$, R. Brock ${ }^{88}$, F. Broggi ${ }^{89 a}$, C. Bromberg ${ }^{88}$, J. Bronner ${ }^{99}$, G. Brooijmans ${ }^{35}$, T. Brooks ${ }^{76}$, W.K. Brooks ${ }^{32 b}$, G. Brown ${ }^{82}$, H. Brown ${ }^{8}$, P.A. Bruckman de Renstrom ${ }^{39}$, D. Bruncko ${ }^{144 b}$,

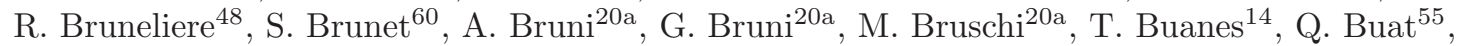
F. Bucci ${ }^{49}$, J. Buchanan ${ }^{118}$, P. Buchholz ${ }^{141}$, R.M. Buckingham ${ }^{118}$, A.G. Buckley ${ }^{46}$, S.I. Buda ${ }^{26 a}$, I.A. Budagov ${ }^{64}$, B. Budick ${ }^{108}$, V. Büscher ${ }^{81}$, L. Bugge ${ }^{117}$, O. Bulekov ${ }^{96}$, A.C. Bundock ${ }^{73}$, M. Bunse ${ }^{43}$, T. Buran ${ }^{117}$, H. Burckhart ${ }^{30}$, S. Burdin ${ }^{73}$, T. Burgess ${ }^{14}$, S. Burke ${ }^{129}$, E. Busato ${ }^{34}$, P. Bussey ${ }^{53}$, C.P. Buszello ${ }^{166}$, B. Butler ${ }^{143}$, J.M. Butler ${ }^{22}$, C.M. Buttar ${ }^{53}$, J.M. Butterworth ${ }^{77}$, W. Buttinger ${ }^{28}$, M. Byszewski ${ }^{30}$, S. Cabrera Urbán ${ }^{167}$, D. Caforio ${ }^{20 a, 20 b}$, O. Cakir ${ }^{4 a}$,

P. Calafiura ${ }^{15}$, G. Calderini ${ }^{78}$, P. Calfayan ${ }^{98}$, R. Calkins ${ }^{106}$, L.P. Caloba ${ }^{24 a}$, R. Caloi ${ }^{132 a, 132 b}$, D. Calvet ${ }^{34}$, S. Calvet ${ }^{34}$, R. Camacho Toro ${ }^{34}$, P. Camarri133a,133b, D. Cameron ${ }^{117}$,

L.M. Caminada ${ }^{15}$, R. Caminal Armadans ${ }^{12}$, S. Campana ${ }^{30}$, M. Campanelli ${ }^{77}$, V. Canale ${ }^{102 a, 102 b}$, F. Canelli ${ }^{31, g}$, A. Canepa ${ }^{159 a}$, J. Cantero ${ }^{80}$, R. Cantrill ${ }^{76}$, L. Capasso ${ }^{102 a, 102 b}$,

M.D.M. Capeans Garrido ${ }^{30}$, I. Caprini ${ }^{26 a}$, M. Caprini ${ }^{26 a}$, D. Capriotti ${ }^{99}$, M. Capua ${ }^{37 a, 37 b}$, R. Caputo $^{81}$, R. Cardarelli ${ }^{133 a}$, T. Carli ${ }^{30}$, G. Carlino ${ }^{102 a}$, L. Carminati ${ }^{89 a, 89 b}$, B. Caron ${ }^{85}$, S. Caron ${ }^{104}$, E. Carquin ${ }^{32 b}$, G.D. Carrillo-Montoya ${ }^{145 b}$, A.A. Carter ${ }^{75}$, J.R. Carter ${ }^{28}$, J. Carvalho ${ }^{124 a, h}$, D. Casadei ${ }^{108}$, M.P. Casado ${ }^{12}$, M. Cascella ${ }^{122 a, 122 b}$, C. Caso $^{50 a, 50 b, *}$, A.M. Castaneda Hernandez ${ }^{173, i}$, E. Castaneda-Miranda ${ }^{173}$, V. Castillo Gimenez ${ }^{167}$, N.F. Castro ${ }^{124 a}$, G. Cataldi ${ }^{72 a}$, P. Catastini ${ }^{57}$, A. Catinaccio ${ }^{30}$, J.R. Catmore ${ }^{30}$, A. Cattai ${ }^{30}$, G. Cattani ${ }^{133 a, 133 b}$, S. Caughron ${ }^{88}$, V. Cavaliere ${ }^{165}$, P. Cavalleri ${ }^{78}$, D. Cavalli ${ }^{89 a}$,

M. Cavalli-Sforza ${ }^{12}$, V. Cavasinni ${ }^{122 \mathrm{a}, 122 \mathrm{~b}}$, F. Ceradini ${ }^{134 a, 134 b}$, A.S. Cerqueira ${ }^{24 b}$, A. Cerri ${ }^{30}$, L. Cerrito ${ }^{75}$, F. Cerutti ${ }^{47}$, S.A. Cetin ${ }^{19 b}$, A. Chafaq ${ }^{135 a}$, D. Chakraborty ${ }^{106}$, I. Chalupkova ${ }^{126}$, K. Chan $^{3}$, P. Chang ${ }^{165}$, B. Chapleau ${ }^{85}$, J.D. Chapman ${ }^{28}$, J.W. Chapman ${ }^{87}$, E. Chareyre ${ }^{78}$, D.G. Charlton ${ }^{18}$, V. Chavda ${ }^{82}$, C.A. Chavez Barajas ${ }^{30}$, S. Cheatham ${ }^{85}$, S. Chekanov ${ }^{6}$, S.V. Chekulaev ${ }^{159 a}$, G.A. Chelkov ${ }^{64}$, M.A. Chelstowska ${ }^{104}$, C. Chen ${ }^{63}$, H. Chen $^{25}$, S. Chen ${ }^{33 c}$, X. Chen ${ }^{173}$, Y. Chen ${ }^{35}$, Y. Cheng ${ }^{31}$, A. Cheplakov ${ }^{64}$, R. Cherkaoui El Moursli ${ }^{135 e}$,

V. Chernyatin ${ }^{25}$, E. Cheu ${ }^{7}$, S.L. Cheung ${ }^{158}$, L. Chevalier ${ }^{136}$, G. Chiefari ${ }^{102 a, 102 b}$,

L. Chikovani ${ }^{51 a}$,*, J.T. Childers ${ }^{30}$, A. Chilingarov ${ }^{71}$, G. Chiodini ${ }^{72 a}$, A.S. Chisholm ${ }^{18}$, R.T. Chislett ${ }^{77}$, A. Chitan ${ }^{26 a}$, M.V. Chizhov ${ }^{64}$, G. Choudalakis ${ }^{31}$, S. Chouridou ${ }^{137}$,

I.A. Christidi 77 , A. Christov ${ }^{48}$, D. Chromek-Burckhart ${ }^{30}$, M.L. Chu ${ }^{151}$, J. Chudoba ${ }^{125}$,

G. Ciapetti ${ }^{132 a, 132 b}$, A.K. Ciftci ${ }^{4 a}$, R. Ciftci ${ }^{4 a}$, D. Cinca ${ }^{34}$, V. Cindro ${ }^{74}$, C. Ciocca $^{20 a}, 20 b$,

A. Ciocio $^{15}$, M. Cirilli ${ }^{87}$, P. Cirkovic ${ }^{13 b}$, Z.H. Citron ${ }^{172}$, M. Citterio ${ }^{89 a}$, M. Ciubancan ${ }^{26 a}$,

A. Clark $^{49}$, P.J. Clark ${ }^{46}$, R.N. Clarke ${ }^{15}$, W. Cleland ${ }^{123}$, J.C. Clemens ${ }^{83}$, B. Clement ${ }^{55}$,

C. Clement ${ }^{146 a, 146 b}$, Y. Coadou ${ }^{83}$, M. Cobal ${ }^{164 a, 164 c}$, A. Coccaro ${ }^{138}$, J. Cochran ${ }^{63}$, L. Coffey ${ }^{23}$, J.G. Cogan $^{143}$, J. Coggeshall ${ }^{165}$, E. Cogneras ${ }^{178}$, J. Colas ${ }^{5}$, S. Cole ${ }^{106}$, A.P. Colijn ${ }^{105}$, N.J. Collins ${ }^{18}$, C. Collins-Tooth ${ }^{53}$, J. Collot ${ }^{55}$, T. Colombo ${ }^{119 a, 119 b}$, G. Colon ${ }^{84}$, G. Compostella ${ }^{99}$, P. Conde Muiño ${ }^{124 a}$, E. Coniavitis ${ }^{166}$, M.C. Conidi ${ }^{12}$, S.M. Consonni ${ }^{89 a, 89 b}$, V. Consorti ${ }^{48}$, S. Constantinescu ${ }^{26 a}$, C. Conta ${ }^{119 a, 119 b}$, G. Conti ${ }^{57}$, F. Conventi102a,j, M. Cooke ${ }^{15}$, B.D. Cooper ${ }^{77}$, A.M. Cooper-Sarkar ${ }^{118}$, K. Copic $^{15}$, T. Cornelissen $^{175}$, M. Corradi ${ }^{20 a}$, F. Corriveau ${ }^{85, k}$, A. Cortes-Gonzalez ${ }^{165}$, G. Cortiana ${ }^{99}$, G. Costa ${ }^{89 a}$, M.J. Costa ${ }^{167}$,

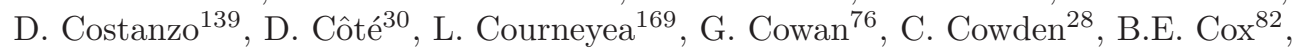
K. Cranmer ${ }^{108}$, F. Crescioli ${ }^{122 a, 122 b}$, M. Cristinziani ${ }^{21}$, G. Crosetti ${ }^{37 a, 37 b}$, S. Crépé-Renaudin ${ }^{55}$, C.-M. Cuciuc ${ }^{26 a}$, C. Cuenca Almenar ${ }^{176}$, T. Cuhadar Donszelmann ${ }^{139}$, M. Curatolo ${ }^{47}$, C.J. Curtis ${ }^{18}$, C. Cuthbert ${ }^{150}$, P. Cwetanski ${ }^{60}$, H. Czirr ${ }^{141}$, P. Czodrowski ${ }^{44}$, Z. Czyczula ${ }^{176}$, S. D'Auria ${ }^{53}$, M. D’Onofrio ${ }^{73}$, A. D'Orazio ${ }^{132 a, 132 b}$, M.J. Da Cunha Sargedas De Sousa ${ }^{124 a}$, C. Da Via ${ }^{82}$, W. Dabrowski ${ }^{38}$, A. Dafinca ${ }^{118}$, T. Dai ${ }^{87}$, C. Dallapiccola ${ }^{84}$, M. Dam ${ }^{36}$, M. Dameri ${ }^{50 a, 50 b}$, D.S. Damiani ${ }^{137}$, H.O. Danielsson ${ }^{30}$, V. Dao ${ }^{49}$, G. Darbo ${ }^{50 a}$, G.L. Darlea ${ }^{26 b}$, 
J.A. Dassoulas ${ }^{42}$, W. Davey ${ }^{21}$, T. Davidek ${ }^{126}$, N. Davidson ${ }^{86}$, R. Davidson ${ }^{71}$, E. Davies ${ }^{118, c}$, M. Davies ${ }^{93}$, O. Davignon ${ }^{78}$, A.R. Davison ${ }^{77}$, Y. Davygora ${ }^{58 a}$, E. Dawe ${ }^{142}$, I. Dawson ${ }^{139}$, R.K. Daya-Ishmukhametova ${ }^{23}$, K. De ${ }^{8}$, R. de Asmundis ${ }^{102 a}$, S. De Castro ${ }^{20 a, 20 b}$, S. De Cecco ${ }^{78}$, J. de Graat ${ }^{98}$, N. De Groot ${ }^{104}$, P. de Jong ${ }^{105}$, C. De La Taille ${ }^{115}$, H. De la Torre ${ }^{80}$, F. De Lorenzi ${ }^{63}$, L. de Mora ${ }^{71}$, L. De Nooij ${ }^{105}$, D. De Pedis ${ }^{132 a}$, A. De Salvo ${ }^{132 a}$, U. De Sanctis ${ }^{164 a, 164 c}$, A. De Santo ${ }^{149}$, J.B. De Vivie De Regie ${ }^{115}$, G. De Zorzi ${ }^{132 a, 132 b}$, W.J. Dearnaley ${ }^{71}$, R. Debbe ${ }^{25}$, C. Debenedetti ${ }^{46}$, B. Dechenaux ${ }^{55}$, D.V. Dedovich ${ }^{64}$, J. Degenhardt ${ }^{120}$, J. Del Peso ${ }^{80}$, T. Del Prete ${ }^{122 \mathrm{a}, 122 \mathrm{~b}}$, T. Delemontex ${ }^{55}$, M. Deliyergiyev ${ }^{74}$, A. Dell'Acqua ${ }^{30}$, L. Dell'Asta ${ }^{22}$, M. Della Pietra ${ }^{102 a, j}$, D. della Volpe ${ }^{102 a, 102 b}$, M. Delmastro ${ }^{5}$, P.A. Delsart ${ }^{55}$, C. Deluca ${ }^{105}$, S. Demers ${ }^{176}$, M. Demichev ${ }^{64}$, B. Demirkoz ${ }^{12, l}$, S.P. Denisov ${ }^{128}$,

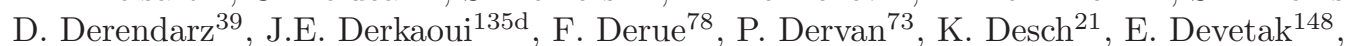
P.O. Deviveiros ${ }^{105}$, A. Dewhurst ${ }^{129}$, B. DeWilde ${ }^{148}$, S. Dhaliwal ${ }^{158}$, R. Dhullipudi ${ }^{25, m}$,

A. Di Ciaccio ${ }^{133 a, 133 b}$, L. Di Ciaccio ${ }^{5}$, C. Di Donato ${ }^{102 a, 102 b}$, A. Di Girolamo ${ }^{30}$, B. Di Girolamo ${ }^{30}$, S. Di Luise ${ }^{134 a, 134 b}$, A. Di Mattia ${ }^{173}$, B. Di Micco ${ }^{30}$, R. Di Nardo ${ }^{47}$, A. Di Simone ${ }^{133 a, 133 b}$, R. Di Sipio ${ }^{20 a}$,20b , M.A. Diaz ${ }^{32 a}$, E.B. Diehl ${ }^{87}$, J. Dietrich ${ }^{42}$, T.A. Dietzsch ${ }^{58 a}$, S. Diglio ${ }^{86}$,

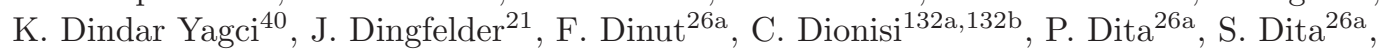
F. Dittus ${ }^{30}$, F. Djama ${ }^{83}$, T. Djobava ${ }^{51 b}$, M.A.B. do Vale ${ }^{24 c}$, A. Do Valle Wemans ${ }^{124 a, n}$, T.K.O. Doan ${ }^{5}$, M. Dobbs ${ }^{85}$, D. Dobos ${ }^{30}$, E. Dobson ${ }^{30, o}$, J. Dodd ${ }^{35}$, C. Doglioni ${ }^{49}$, T. Doherty ${ }^{53}$, Y. Doi ${ }^{65, *}$, J. Dolejsi ${ }^{126}$, I. Dolenc ${ }^{74}$, Z. Dolezal ${ }^{126}$, B.A. Dolgoshein ${ }^{96, *}$, T. Dohmae ${ }^{155}$, M. Donadellii ${ }^{24}$, J. Donini ${ }^{34}$, J. Dopke ${ }^{30}$, A. Doria ${ }^{102 a}$, A. Dos Anjos ${ }^{173}$, A. Dotti $^{122 a, 122 b}$, M.T. Dova ${ }^{70}$, A.D. Doxiadis ${ }^{105}$, A.T. Doyle ${ }^{53}$, N. Dressnandt ${ }^{120}$, M. Dris ${ }^{10}$, J. Dubbert ${ }^{99}$, S. Dube ${ }^{15}$, E. Duchovni ${ }^{172}$, G. Duckeck ${ }^{98}$, D. Duda ${ }^{175}$, A. Dudarev ${ }^{30}$, F. Dudziak ${ }^{63}$, M. Dührssen ${ }^{30}$, I.P. Duerdoth ${ }^{82}$, L. Duflot ${ }^{115}$, M-A. Dufour ${ }^{85}$, L. Duguid ${ }^{76}$, M. Dunford ${ }^{58 a}$, H. Duran Yildiz ${ }^{4 a}$, R. Duxfield ${ }^{139}$, M. Dwuznik ${ }^{38}$, M. Düren ${ }^{52}$, W.L. Ebenstein ${ }^{45}$, J. Ebke ${ }^{98}$, S. Eckweiler ${ }^{81}$, K. Edmonds ${ }^{81}$, W. Edson ${ }^{2}$, C.A. Edwards ${ }^{76}$, N.C. Edwards ${ }^{53}$, W. Ehrenfeld ${ }^{42}$, T. Eifert ${ }^{143}$, G. Eigen ${ }^{14}$, K. Einsweiler ${ }^{15}$, E. Eisenhandler ${ }^{75}$, T. Ekelof ${ }^{166}$, M. El Kacimi ${ }^{135 c}$, M. Ellert ${ }^{166}$, S. Elles ${ }^{5}$, F. Ellinghaus ${ }^{81}$, K. Ellis ${ }^{75}$, N. Ellis $^{30}$, J. Elmsheuser ${ }^{98}$, M. Elsing ${ }^{30}$, D. Emeliyanov ${ }^{129}$, R. Engelmann ${ }^{148}$, A. Engl ${ }^{98}$, B. $\operatorname{Epp}^{61}$, J. Erdmann ${ }^{54}$, A. Ereditato ${ }^{17}$, D. Eriksson ${ }^{146 a}$, J. Ernst ${ }^{2}$, M. Ernst ${ }^{25}$, J. Ernwein ${ }^{136}$, D. Errede ${ }^{165}$, S. Errede ${ }^{165}$, E. Ertel ${ }^{81}$, M. Escalier ${ }^{115}$, H. Esch ${ }^{43}$, C. Escobar ${ }^{123}$, X. Espinal Curull ${ }^{12}$, B. Esposito ${ }^{47}$, F. Etienne ${ }^{83}$, A.I. Etienvre ${ }^{136}$, E. Etzion ${ }^{153}$, D. Evangelakou ${ }^{54}$, H. Evans $^{60}$, L. Fabbri ${ }^{20 a, 20 b}$, C. Fabre $^{30}$, R.M. Fakhrutdinov ${ }^{128}$, S. Falciano ${ }^{132 a}$, Y. Fang ${ }^{173}$, M. Fanti89a,89b , A. Farbin ${ }^{8}$, A. Farilla ${ }^{134 a}$, J. Farley ${ }^{148}$, T. Farooque ${ }^{158}$, S. Farrell ${ }^{163}$, S.M. Farrington ${ }^{170}$, P. Farthouat ${ }^{30}$, F. Fassi ${ }^{167}$, P. Fassnacht ${ }^{30}$, D. Fassouliotis ${ }^{9}$, B. Fatholahzadeh ${ }^{158}$, A. Favareto ${ }^{89 a}, 89 \mathrm{~b}$, L. Fayard $^{115}$, S. Fazio ${ }^{37 a, 37 b}$, R. Febbraro ${ }^{34}$, P. Federic ${ }^{144 a}$, O.L. Fedin ${ }^{121}$, W. Fedorko ${ }^{88}$, M. Fehling-Kaschek ${ }^{48}$, L. Feligioni ${ }^{83}$, C. Feng ${ }^{33 d}$, E.J. Feng ${ }^{6}$, A.B. Fenyuk ${ }^{128}$, J. Ferencei ${ }^{144 b}$, W. Fernando ${ }^{6}$, S. Ferrag ${ }^{53}$, J. Ferrando ${ }^{53}$, V. Ferrara ${ }^{42}$, A. Ferrari ${ }^{166}$, P. Ferrari ${ }^{105}$, R. Ferrari119a, D.E. Ferreira de Lima ${ }^{53}$, A. Ferrer ${ }^{167}$, D. Ferrere ${ }^{49}$, C. Ferretti ${ }^{87}$, A. Ferretto Parodi ${ }^{50 a, 50 b}$, M. Fiascaris ${ }^{31}$, F. Fiedler ${ }^{81}$, A. Filipčič ${ }^{74}$, F. Filthaut ${ }^{104}$, M. Fincke-Keeler ${ }^{169}$, M.C.N. Fiolhais ${ }^{124 a}, h$, L. Fiorini ${ }^{167}$, A. Firan ${ }^{40}$, G. Fischer ${ }^{42}$, M.J. Fisher ${ }^{109}$, M. Flechl ${ }^{48}$, I. Fleck ${ }^{141}$, J. Fleckner ${ }^{81}$, P. Fleischmann ${ }^{174}$, S. Fleischmann ${ }^{175}$, T. Flick ${ }^{175}$, A. Floderus ${ }^{79}$, L.R. Flores Castillo ${ }^{173}$, M.J. Flowerdew ${ }^{99}$, T. Fonseca Martin ${ }^{17}$, A. Formica ${ }^{136}$, A. Forti ${ }^{82}$, D. Fortin ${ }^{159 a}$, D. Fournier ${ }^{115}$, A.J. Fowler ${ }^{45}$, H. Fox ${ }^{71}$, P. Francavilla ${ }^{12}$, M. Franchini ${ }^{20 a, 20 b}$, S. Franchino ${ }^{119 a, 119 b}$, D. Francis ${ }^{30}$, T. Frank ${ }^{172}$, M. Franklin ${ }^{57}$, S. Franz ${ }^{30}$, M. Fraternali ${ }^{119 a, 119 b}$, S. Fratina ${ }^{120}$, S.T. French ${ }^{28}$, C. Friedrich ${ }^{42}$, F. Friedrich ${ }^{44}$, R. Froeschl ${ }^{30}$, D. Froidevaux ${ }^{30}$, J.A. Frost ${ }^{28}$, C. Fukunaga ${ }^{156}$,

E. Fullana Torregrosa ${ }^{30}$, B.G. Fulsom ${ }^{143}$, J. Fuster ${ }^{167}$, C. Gabaldon ${ }^{30}$, O. Gabizon ${ }^{172}$, T. Gadfort ${ }^{25}$, S. Gadomski ${ }^{49}$, G. Gagliardi ${ }^{50 a}$,50b, P. Gagnon ${ }^{60}$, C. Galea ${ }^{98}$, B. Galhardo ${ }^{124 a}$, E.J. Gallas ${ }^{118}$, V. Gallo ${ }^{17}$, B.J. Gallop ${ }^{129}$, P. Gallus ${ }^{125}$, K.K. Gan ${ }^{109}$, Y.S. Gao ${ }^{143, e}$,

A. Gaponenko ${ }^{15}$, F. Garberson ${ }^{176}$, M. Garcia-Sciveres ${ }^{15}$, C. García ${ }^{167}$, J.E. García Navarro ${ }^{167}$, R.W. Gardner ${ }^{31}$, N. Garelli ${ }^{30}$, H. Garitaonandia ${ }^{105}$, V. Garonne ${ }^{30}$, C. Gatti ${ }^{47}$, G. Gaudio ${ }^{119 a}$, B. Gaur ${ }^{141}$, L. Gauthier ${ }^{136}$, P. Gauzzi ${ }^{132 a, 132 b}$, I.L. Gavrilenko ${ }^{94}$, C. Gay ${ }^{168}$, G. Gaycken ${ }^{21}$, E.N. Gazis ${ }^{10}$, P. Ge ${ }^{33 d}$, Z. Gecse ${ }^{168}$, C.N.P. Gee ${ }^{129}$, D.A.A. Geerts ${ }^{105}$, Ch. Geich-Gimbel ${ }^{21}$, 
K. Gellerstedt ${ }^{146 a, 146 b}$, C. Gemme ${ }^{50 a}$, A. Gemmell ${ }^{53}$, M.H. Genest ${ }^{55}$, S. Gentile ${ }^{132 a, 132 b}$, M. George ${ }^{54}$, S. George ${ }^{76}$, P. Gerlach ${ }^{175}$, A. Gershon ${ }^{153}$, C. Geweniger ${ }^{58 \mathrm{a}}$, H. Ghazlane ${ }^{135 \mathrm{~b}}$, N. Ghodbane ${ }^{34}$, B. Giacobbe ${ }^{20 a}$, S. Giagu ${ }^{132 a, 132 b}$, V. Giakoumopoulou ${ }^{9}$, V. Giangiobbe ${ }^{12}$, F. Gianotti ${ }^{30}$, B. Gibbard ${ }^{25}$, A. Gibson ${ }^{158}$, S.M. Gibson ${ }^{30}$, M. Gilchriese ${ }^{15}$, D. Gillberg ${ }^{29}$, A.R. Gillman ${ }^{129}$, D.M. Gingrich ${ }^{3, d}$, J. Ginzburg ${ }^{153}$, N. Giokaris ${ }^{9}$, M.P. Giordani ${ }^{164 c}$,

R. Giordano ${ }^{102 a, 102 b}$, F.M. Giorgi ${ }^{16}$, P. Giovannini ${ }^{99}$, P.F. Giraud ${ }^{136}$, D. Giugni ${ }^{89 a}$, M. Giunta ${ }^{93}$, B.K. Gjelsten ${ }^{117}$, L.K. Gladilin ${ }^{97}$, C. Glasman ${ }^{80}$, J. Glatzer ${ }^{21}$, A. Glazov ${ }^{42}$, K.W. Glitza ${ }^{175}$, G.L. Glonti ${ }^{64}$, J.R. Goddard ${ }^{75}$, J. Godfrey ${ }^{142}$, J. Godlewski ${ }^{30}$, M. Goebel ${ }^{42}$, T. Göpfert ${ }^{44}$, C. Goeringer ${ }^{81}$, C. Gössling ${ }^{43}$, S. Goldfarb ${ }^{87}$, T. Golling ${ }^{176}$, A. Gomes ${ }^{124 a, b}$,

L.S. Gomez Fajardo ${ }^{42}$, R. Gonçalo ${ }^{76}$, J. Goncalves Pinto Firmino Da Costa ${ }^{42}$, L. Gonella ${ }^{21}$, S. González de la Hoz ${ }^{167}$, G. Gonzalez Parra ${ }^{12}$, M.L. Gonzalez Silva ${ }^{27}$, S. Gonzalez-Sevilla ${ }^{49}$, J.J. Goodson ${ }^{148}$, L. Goossens ${ }^{30}$, P.A. Gorbounov ${ }^{95}$, H.A. Gordon ${ }^{25}$, I. Gorelov ${ }^{103}$, G. Gorfine ${ }^{175}$, B. Gorini ${ }^{30}$, E. Gorini ${ }^{72 a, 72 b}$, A. Gorišek ${ }^{74}$, E. Gornicki ${ }^{39}$, A.T. Goshaw ${ }^{6}$, M. Gosselink ${ }^{105}$, M.I. Gostkin ${ }^{64}$, I. Gough Eschrich ${ }^{163}$, M. Gouighri ${ }^{135 a}$, D. Goujdami ${ }^{135 c}$, M.P. Goulette ${ }^{49}$, A.G. Goussiou ${ }^{138}$, C. Goy ${ }^{5}$, S. Gozpinar ${ }^{23}$, I. Grabowska-Bold ${ }^{38}$, P. Grafström²0a,20b, K-J. Grahn ${ }^{42}$, E. Gramstad ${ }^{117}$, F. Grancagnolo ${ }^{72 a}$, S. Grancagnolo ${ }^{16}$, V. Grassi ${ }^{148}$, V. Gratchev ${ }^{121}$, N. Grau ${ }^{35}$, H.M. Gray ${ }^{30}$, J.A. Gray ${ }^{148}$, E. Graziani ${ }^{134 a}$, O.G. Grebenyuk ${ }^{121}$, T. Greenshaw ${ }^{73}$, Z.D. Greenwood ${ }^{25, m}$, K. Gregersen ${ }^{36}$, I.M. Gregor ${ }^{42}$, P. Grenier ${ }^{143}$, J. Griffiths ${ }^{8}$, N. Grigalashvili ${ }^{64}$, A.A. Grillo ${ }^{137}$, S. Grinstein ${ }^{12}$, Ph. Gris ${ }^{34}$, Y.V. Grishkevich ${ }^{97}$, J.-F. Grivaz ${ }^{115}$, E. Gross ${ }^{172}$, J. Grosse-Knetter ${ }^{54}$, J. Groth-Jensen ${ }^{172}$, K. Grybel ${ }^{141}$, D. Guest ${ }^{176}$, C. Guicheney ${ }^{34}$, E. Guido ${ }^{50 a, 50 b}$, S. Guindon ${ }^{54}$, U. Gul ${ }^{53}$, J. Gunther ${ }^{125}$, B. Guo ${ }^{158}$, J. Guo ${ }^{35}$, P. Gutierrez ${ }^{111}$, N. Guttman ${ }^{153}$, O. Gutzwiller ${ }^{173}$, C. Guyot ${ }^{136}$, C. Gwenlan ${ }^{118}$, C.B. Gwilliam ${ }^{73}$, A. Haas ${ }^{108}$, S. Haas ${ }^{30}$, C. Haber ${ }^{15}$, H.K. Hadavand ${ }^{8}$, D.R. Hadley ${ }^{18}$, P. Haefner ${ }^{21}$, F. Hahn ${ }^{30}$, Z. Hajduk ${ }^{39}$, H. Hakobyan ${ }^{177}$, D. Hall ${ }^{118}$, K. Hamacher ${ }^{175}$, P. Hamal ${ }^{113}$, K. Hamano ${ }^{86}$, M. Hamer ${ }^{54}$, A. Hamilton ${ }^{145 b, p}$, S. Hamilton ${ }^{161}$, L. Han ${ }^{33 b}$, K. Hanagaki ${ }^{116}$, K. Hanawa ${ }^{160}$, M. Hance ${ }^{15}$, C. Handel ${ }^{81}$, P. Hanke ${ }^{58 a}$, J.R. Hansen ${ }^{36}$, J.B. Hansen ${ }^{36}$, J.D. Hansen ${ }^{36}$, P.H. Hansen ${ }^{36}$,

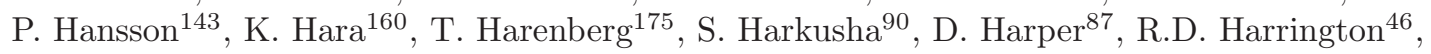
O.M. Harris ${ }^{138}$, J. Hartert ${ }^{48}$, F. Hartjes ${ }^{105}$, T. Haruyama ${ }^{65}$, A. Harvey ${ }^{56}$, S. Hasegawa ${ }^{101}$, Y. Hasegawa ${ }^{140}$, S. Hassani ${ }^{136}$, S. Haug ${ }^{17}$, M. Hauschild ${ }^{30}$, R. Hauser ${ }^{88}$, M. Havranek ${ }^{21}$, C.M. Hawkes ${ }^{18}$, R.J. Hawkings ${ }^{30}$, A.D. Hawkins ${ }^{79}$, T. Hayakawa ${ }^{66}$, T. Hayashi ${ }^{160}$, D. Hayden ${ }^{76}$, C.P. Hays ${ }^{118}$, H.S. Hayward ${ }^{73}$, S.J. Haywood ${ }^{129}$, S.J. Head ${ }^{18}$, V. Hedberg ${ }^{79}$, L. Heelan ${ }^{8}$, S. Heim ${ }^{88}$, B. Heinemann ${ }^{15}$, S. Heisterkamp ${ }^{36}$, L. Helary ${ }^{22}$, C. Heller ${ }^{98}$, M. Heller ${ }^{30}$, S. Hellman ${ }^{146 a, 146 b}$, D. Hellmich ${ }^{21}$, C. Helsens ${ }^{12}$, R.C.W. Henderson ${ }^{71}$, M. Henke ${ }^{58 a}$, A. Henrichs ${ }^{176}$, A.M. Henriques Correia ${ }^{30}$, S. Henrot-Versille ${ }^{115}$, C. Hensel $^{54}$, T. Henß ${ }^{175}$, C.M. Hernandez ${ }^{8}$, Y. Hernández Jiménez ${ }^{167}$, R. Herrberg ${ }^{16}$, G. Herten ${ }^{48}$, R. Hertenberger ${ }^{98}$, L. Hervas ${ }^{30}$, G.G. Hesketh ${ }^{77}$, N.P. Hessey ${ }^{105}$, E. Higón-Rodriguez ${ }^{167}$, J.C. Hill ${ }^{28}$, K.H. Hiller ${ }^{42}$, S. Hillert ${ }^{21}$, S.J. Hillier ${ }^{18}$, I. Hinchliffe ${ }^{15}$, E. Hines ${ }^{120}$, M. Hirose ${ }^{116}$, F. Hirsch ${ }^{43}$, D. Hirschbuehl ${ }^{175}$, J. Hobbs ${ }^{148}$, N. Hod ${ }^{153}$, M.C. Hodgkinson ${ }^{139}$, P. Hodgson ${ }^{139}$, A. Hoecker ${ }^{30}$, M.R. Hoeferkamp ${ }^{103}$, J. Hoffman ${ }^{40}$, D. Hoffmann ${ }^{83}$, M. Hohlfeld ${ }^{81}$, M. Holder ${ }^{141}$, S.O. Holmgren ${ }^{146 a}$, T. Holy ${ }^{127}$, J.L. Holzbauer ${ }^{88}$, T.M. Hong ${ }^{120}$, L. Hooft van Huysduynen ${ }^{108}$,

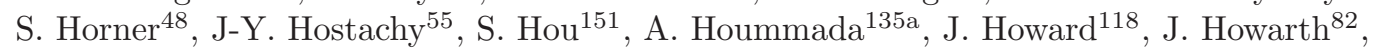
I. Hristova ${ }^{16}$, J. Hrivnac ${ }^{115}$, T. Hryn'ova ${ }^{5}$, P.J. Hsu ${ }^{81}$, S.-C. Hsu ${ }^{15}$, D. Hu ${ }^{35}$, Z. Hubacek ${ }^{127}$, F. Hubaut ${ }^{83}$, F. Huegging ${ }^{21}$, A. Huettmann ${ }^{42}$, T.B. Huffman ${ }^{118}$, E.W. Hughes ${ }^{35}$, G. Hughes ${ }^{71}$, M. Huhtinen ${ }^{30}$, M. Hurwitz ${ }^{15}$, N. Huseynov ${ }^{64, q}$, J. Huston ${ }^{88}$, J. Huth ${ }^{57}$, G. Iacobucci ${ }^{49}$, G. Iakovidis ${ }^{10}$, M. Ibbotson ${ }^{82}$, I. Ibragimov ${ }^{141}$, L. Iconomidou-Fayard ${ }^{115}$, J. Idarraga ${ }^{115}$,

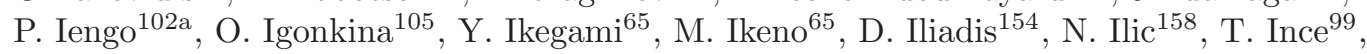
P. Ioannou ${ }^{9}$, M. Iodice ${ }^{134 a}$, K. Iordanidou ${ }^{9}$, V. Ippolito ${ }^{132 a, 132 b}$, A. Irles Quiles ${ }^{167}$, C. Isaksson ${ }^{166}$, M. Ishino ${ }^{67}$, M. Ishitsuka ${ }^{157}$, R. Ishmukhametov ${ }^{109}$, C. Issever ${ }^{118}$, S. Istin ${ }^{19 a}$, A.V. Ivashin ${ }^{128}$, W. Iwanski ${ }^{39}$, H. Iwasaki ${ }^{65}$, J.M. Izen ${ }^{41}$, V. Izzo ${ }^{102 a}$, B. Jackson ${ }^{120}$, J.N. Jackson ${ }^{73}$, P. Jackson ${ }^{1}$, M.R. Jaekel ${ }^{30}$, V. Jain ${ }^{60}$, K. Jakobs ${ }^{48}$, S. Jakobsen ${ }^{36}$, T. Jakoubek ${ }^{125}$, J. Jakubek ${ }^{127}$,

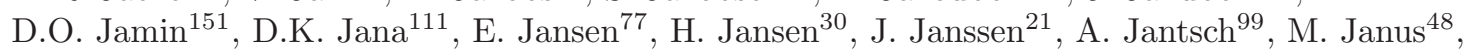
G. Jarlskog ${ }^{79}$, L. Jeanty ${ }^{57}$, I. Jen-La Plante ${ }^{31}$, D. Jennens ${ }^{86}$, P. Jenni ${ }^{30}$, A.E. Loevschall-Jensen ${ }^{36}$, 


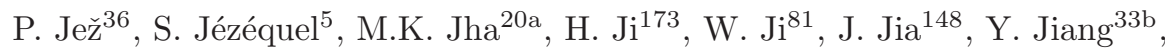

M. Jimenez Belenguer ${ }^{42}$, S. Jin ${ }^{33 a}$, O. Jinnouchi ${ }^{157}$, M.D. Joergensen ${ }^{36}$, D. Joffe ${ }^{40}$,

M. Johansen ${ }^{146 a, 146 b}$, K.E. Johansson ${ }^{146 a}$, P. Johansson ${ }^{139}$, S. Johnert ${ }^{42}$, K.A. Johns ${ }^{7}$,

K. Jon-And ${ }^{146 a, 146 b}$, G. Jones ${ }^{170}$, R.W.L. Jones ${ }^{71}$, T.J. Jones ${ }^{73}$, C. Joram ${ }^{30}$, P.M. Jorge ${ }^{124 a}$,

K.D. Joshi ${ }^{82}$, J. Jovicevic ${ }^{147}$, T. Jovin ${ }^{13 b}$, X. Ju ${ }^{173}$, C.A. Jung ${ }^{43}$, R.M. Jungst ${ }^{30}$, V. Juranek ${ }^{125}$, P. Jussel ${ }^{61}$, A. Juste Rozas ${ }^{12}$, S. Kabana ${ }^{17}$, M. Kaci ${ }^{167}$, A. Kaczmarska ${ }^{39}$, P. Kadlecik ${ }^{36}$,

M. Kado ${ }^{115}$, H. Kagan ${ }^{109}$, M. Kagan ${ }^{57}$, E. Kajomovitz ${ }^{152}$, S. Kalinin ${ }^{175}$, L.V. Kalinovskaya ${ }^{64}$,

S. Kama ${ }^{40}$, N. Kanaya ${ }^{155}$, M. Kaneda ${ }^{30}$, S. Kaneti ${ }^{28}$, T. Kanno ${ }^{157}$, V.A. Kantserov ${ }^{96}$,

J. Kanzaki ${ }^{65}$, B. Kaplan ${ }^{108}$, A. Kapliy ${ }^{31}$, J. Kaplon ${ }^{30}$, D. Kar ${ }^{53}$, M. Karagounis ${ }^{21}$,

K. Karakostas ${ }^{10}$, M. Karnevskiy ${ }^{42}$, V. Kartvelishvili ${ }^{71}$, A.N. Karyukhin ${ }^{128}$, L. Kashif ${ }^{173}$,

G. Kasieczka ${ }^{58 b}$, R.D. Kass ${ }^{109}$, A. Kastanas ${ }^{14}$, M. Kataoka ${ }^{5}$, Y. Kataoka ${ }^{155}$, E. Katsoufis ${ }^{10}$,

J. Katzy ${ }^{42}$, V. Kaushik ${ }^{7}$, K. Kawagoe ${ }^{69}$, T. Kawamoto ${ }^{155}$, G. Kawamura ${ }^{81}$, M.S. Kayl ${ }^{105}$,

S. Kazama ${ }^{155}$, V.A. Kazanin ${ }^{107}$, M.Y. Kazarinov ${ }^{64}$, R. Keeler ${ }^{169}$, P.T. Keener ${ }^{120}$, R. Kehoe ${ }^{40}$,

M. Keil ${ }^{54}$, G.D. Kekelidze ${ }^{64}$, J.S. Keller ${ }^{138}$, M. Kenyon ${ }^{53}$, O. Kepka ${ }^{125}$, N. Kerschen ${ }^{30}$,

B.P. Kerševan ${ }^{74}$, S. Kersten ${ }^{175}$, K. Kessoku ${ }^{155}$, J. Keung ${ }^{158}$, F. Khalil-zada ${ }^{11}$,

H. Khandanyan ${ }^{146 a, 146 b}$, A. Khanov ${ }^{112}$, D. Kharchenko ${ }^{64}$, A. Khodinov ${ }^{96}$, A. Khomich ${ }^{58 a}$,

T.J. Khoo ${ }^{28}$, G. Khoriauli ${ }^{21}$, A. Khoroshilov ${ }^{175}$, V. Khovanskiy ${ }^{95}$, E. Khramov ${ }^{64}$, J. Khubua ${ }^{51 b}$,

H. Kim ${ }^{146 a, 146 b}$, S.H. Kim ${ }^{160}$, N. Kimura ${ }^{171}$, O. Kind ${ }^{16}$, B.T. King ${ }^{73}$, M. King ${ }^{66}$, R.S.B. King ${ }^{118}$,

J. Kirk ${ }^{129}$, A.E. Kiryunin ${ }^{99}$, T. Kishimoto ${ }^{66}$, D. Kisielewska ${ }^{38}$, T. Kitamura ${ }^{66}$, T. Kittelmann ${ }^{123}$,

K. Kiuchi ${ }^{160}$, E. Kladiva ${ }^{144 b}$, M. Klein ${ }^{73}$, U. Klein ${ }^{73}$, K. Kleinknecht ${ }^{81}$, M. Klemetti ${ }^{85}$,

A. Klier ${ }^{172}$, P. Klimek ${ }^{146 a, 146 b}$, A. Klimentov ${ }^{25}$, R. Klingenberg ${ }^{43}$, J.A. Klinger ${ }^{82}$, E.B. Klinkby ${ }^{36}$,

T. Klioutchnikova ${ }^{30}$, P.F. Klok ${ }^{104}$, S. Klous ${ }^{105}$, E.-E. Kluge ${ }^{58 a}$, T. Kluge ${ }^{73}$, P. Kluit ${ }^{105}$,

S. Kluth ${ }^{99}$, E. Kneringer ${ }^{61}$, E.B.F.G. Knoops ${ }^{83}$, A. Knue ${ }^{54}$, B.R. Ko ${ }^{45}$, T. Kobayashi ${ }^{155}$,

M. Kobel ${ }^{44}$, M. Kocian ${ }^{143}$, P. Kodys ${ }^{126}$, K. Köneke ${ }^{30}$, A.C. König ${ }^{104}$, S. Koenig ${ }^{81}$, L. Köpke ${ }^{81}$,

F. Koetsveld ${ }^{104}$, P. Koevesarki ${ }^{21}$, T. $\operatorname{Koffas}^{29}$, E. Koffeman ${ }^{105}$, L.A. Kogan ${ }^{118}$, S. Kohlmann ${ }^{175}$,

F. Kohn ${ }^{54}$, Z. Kohout ${ }^{127}$, T. Kohriki ${ }^{65}$, T. Koi ${ }^{143}$, G.M. Kolachev ${ }^{107, *}$, H. Kolanoski ${ }^{16}$,

V. Kolesnikov ${ }^{64}$, I. Koletsou ${ }^{89 a}$, J. Koll ${ }^{88}$, A.A. Komar ${ }^{94}$, Y. Komori ${ }^{155}$, T. Kondo ${ }^{65}$, T. Kono ${ }^{42, r}$, A.I. Kononov ${ }^{48}$, R. Konoplich ${ }^{108, s}$, N. Konstantinidis ${ }^{77}$, R. Kopeliansky ${ }^{152}$, S. Koperny ${ }^{38}$,

K. Korcyl ${ }^{39}$, K. Kordas ${ }^{154}$, A. Korn ${ }^{118}$, A. Korol ${ }^{107}$, I. Korolkov ${ }^{12}$, E.V. Korolkova ${ }^{139}$,

V.A. Korotkov ${ }^{128}$, O. Kortner ${ }^{99}$, S. Kortner ${ }^{99}$, V.V. Kostyukhin ${ }^{21}$, S. Kotov ${ }^{99}$, V.M. Kotov ${ }^{64}$,

A. Kotwal ${ }^{45}$, C. Kourkoumelis ${ }^{9}$, V. Kouskoura ${ }^{154}$, A. Koutsman ${ }^{159 a}$, R. Kowalewski ${ }^{169}$,

T.Z. Kowalski ${ }^{38}$, W. Kozanecki ${ }^{136}$, A.S. Kozhin ${ }^{128}$, V. Kral ${ }^{127}$, V.A. Kramarenko ${ }^{97}$,

G. Kramberger ${ }^{74}$, M.W. Krasny ${ }^{78}$, A. Krasznahorkay ${ }^{108}$, J.K. Kraus ${ }^{21}$, S. Kreiss ${ }^{108}$, F. Krejci ${ }^{127}$,

J. Kretzschmar ${ }^{73}$, N. Krieger ${ }^{54}$, P. Krieger ${ }^{158}$, K. Kroeninger ${ }^{54}$, H. Kroha ${ }^{99}$, J. Kroll ${ }^{120}$,

J. Kroseberg ${ }^{21}$, J. Krstic ${ }^{13 a}$, U. Kruchonak ${ }^{64}$, H. Krüger ${ }^{21}$, T. Kruker ${ }^{17}$, N. Krumnack ${ }^{63}$,

Z.V. Krumshteyn ${ }^{64}$, M.K. Kruse ${ }^{45}$, T. Kubota ${ }^{86}$, S. Kuday ${ }^{4 a}$, S. Kuehn ${ }^{48}$, A. Kugel ${ }^{58 c}$, T. Kuhl ${ }^{42}$,

D. Kuhn ${ }^{61}$, V. Kukhtin ${ }^{64}$, Y. Kulchitsky ${ }^{90}$, S. Kuleshov ${ }^{32 b}$, C. Kummer ${ }^{98}$, M. Kuna ${ }^{78}$,

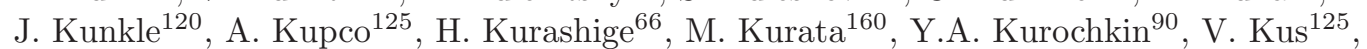

E.S. Kuwertz ${ }^{147}$, M. Kuze ${ }^{157}$, J. Kvita ${ }^{142}$, R. Kwee ${ }^{16}$, A. La Rosa ${ }^{49}$, L. La Rotonda ${ }^{37 a, 37 b}$,

L. Labarga ${ }^{80}$, J. Labbe ${ }^{5}$, S. Lablak ${ }^{135 a}$, C. Lacasta ${ }^{167}$, F. Lacava ${ }^{132 a, 132 b}$, J. Lacey ${ }^{29}$, H. Lacker $^{16}$,

D. Lacour ${ }^{78}$, V.R. Lacuesta ${ }^{167}$, E. Ladygin ${ }^{64}$, R. Lafaye ${ }^{5}$, B. Laforge ${ }^{78}$, T. Lagouri ${ }^{176}$, S. Lai $^{48}$,

E. Laisne ${ }^{55}$, L. Lambourne ${ }^{77}$, C.L. Lampen ${ }^{7}$, W. Lampl ${ }^{7}$, E. Lancon ${ }^{136}$, U. Landgraf ${ }^{48}$,

M.P.J. Landon ${ }^{75}$, V.S. Lang ${ }^{58 a}$, C. Lange ${ }^{42}$, A.J. Lankford ${ }^{163}$, F. Lanni ${ }^{25}$, K. Lantzsch ${ }^{175}$,

S. Laplace ${ }^{78}$, C. Lapoire ${ }^{21}$, J.F. Laporte ${ }^{136}$, T. Lari ${ }^{89 a}$, A. Larner ${ }^{118}$, M. Lassnig ${ }^{30}$, P. Laurelli ${ }^{47}$,

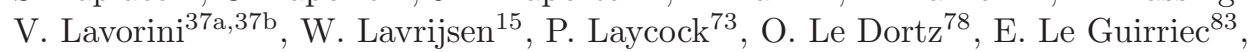

E. Le Menedeu ${ }^{12}$, T. LeCompte ${ }^{6}$, F. Ledroit-Guillon ${ }^{55}$, H. Lee ${ }^{105}$, J.S.H. Lee ${ }^{116}$, S.C. Lee ${ }^{151}$,

L. Lee ${ }^{176}$, M. Lefebvre ${ }^{169}$, M. Legendre ${ }^{136}$, F. Legger ${ }^{98}$, C. Leggett ${ }^{15}$, M. Lehmacher $^{21}$,

G. Lehmann Miotto ${ }^{30}$, X. Lei ${ }^{7}$, M.A.L. Leite ${ }^{24 d}$, R. Leitner ${ }^{126}$, D. Lellouch ${ }^{172}$, B. Lemmer ${ }^{54}$,

V. Lendermann ${ }^{58 a}$, K.J.C. Leney ${ }^{145 b}$, T. Lenz ${ }^{105}$, G. Lenzen ${ }^{175}$, B. Lenzi ${ }^{30}$, K. Leonhardt ${ }^{44}$,

S. Leontsinis ${ }^{10}$, F. Lepold ${ }^{58 a}$, C. Leroy ${ }^{93}$, J-R. Lessard ${ }^{169}$, C.G. Lester ${ }^{28}$, C.M. Lester ${ }^{120}$,

J. Levêque ${ }^{5}$, D. Levin ${ }^{87}$, L.J. Levinson ${ }^{172}$, A. Lewis ${ }^{118}$, G.H. Lewis ${ }^{108}$, A.M. Leyko ${ }^{21}$,

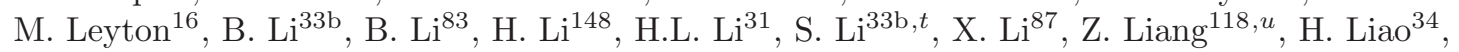




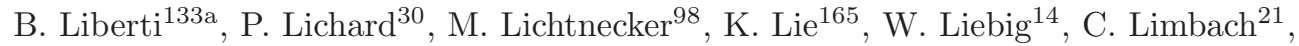

A. Limosani ${ }^{86}$, M. Limper ${ }^{62}$, S.C. Lin ${ }^{151, v}$, F. Linde ${ }^{105}$, J.T. Linnemann ${ }^{88}$, E. Lipeles ${ }^{120}$,

A. Lipniacka ${ }^{14}$, T.M. Liss ${ }^{165}$, D. Lissauer ${ }^{25}$, A. Lister ${ }^{49}$, A.M. Litke ${ }^{137}$, C. Liu ${ }^{29}$, D. Liu ${ }^{151}$,

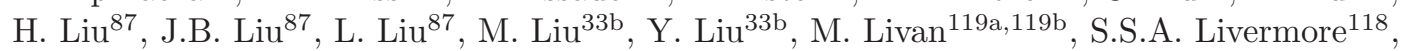

A. Lleres ${ }^{55}$, J. Llorente Merino ${ }^{80}$, S.L. Lloyd ${ }^{75}$, E. Lobodzinska ${ }^{42}$, P. Loch ${ }^{7}$, W.S. Lockman ${ }^{137}$,

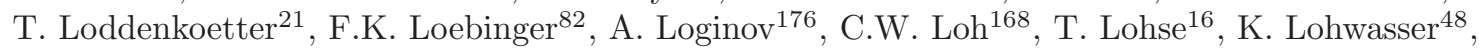

M. Lokajicek ${ }^{125}$, V.P. Lombardo ${ }^{5}$, R.E. Long ${ }^{71}$, L. Lopes ${ }^{124 a}$, D. Lopez Mateos ${ }^{57}$, J. Lorenz ${ }^{98}$,

N. Lorenzo Martinez ${ }^{115}$, M. Losada ${ }^{162}$, P. Loscutoff ${ }^{15}$, F. Lo Sterzo ${ }^{132 a, 132 b}$, M.J. Losty ${ }^{159 a, *}$,

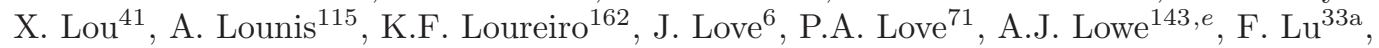

H.J. Lubatti ${ }^{138}$, C. Luci ${ }^{132 a, 132 b}$, A. Lucotte ${ }^{55}$, A. Ludwig ${ }^{44}$, D. Ludwig ${ }^{42}$, I. Ludwig ${ }^{48}$,

J. Ludwig ${ }^{48}$, F. Luehring ${ }^{60}$, G. Luijckx ${ }^{105}$, W. Lukas ${ }^{61}$, L. Luminari ${ }^{132 a}$, E. Lund ${ }^{117}$,

B. Lund-Jensen ${ }^{147}$, B. Lundberg ${ }^{79}$, J. Lundberg 146a,146b , O. Lundberg 146a,146b, J. Lundquist ${ }^{36}$,

M. Lungwitz ${ }^{81}$, D. Lynn ${ }^{25}$, E. Lytken ${ }^{79}$, H. Ma ${ }^{25}$, L.L. Ma ${ }^{173}$, G. Maccarrone ${ }^{47}$, A. Macchiolo ${ }^{99}$,

B. Maček ${ }^{74}$, J. Machado Miguens ${ }^{124 a}$, D. Macina ${ }^{30}$, R. Mackeprang ${ }^{36}$, R.J. Madaras ${ }^{15}$,

H.J. Maddocks ${ }^{71}$, W.F. Mader ${ }^{44}$, R. Maenner ${ }^{58 c}$, T. Maeno ${ }^{25}$, P. Mättig ${ }^{175}$, S. Mättig ${ }^{42}$,

L. Magnoni ${ }^{163}$, E. Magradze ${ }^{54}$, K. Mahboubi ${ }^{48}$, J. Mahlstedt ${ }^{105}$, S. Mahmoud ${ }^{73}$, G. Mahout ${ }^{18}$,

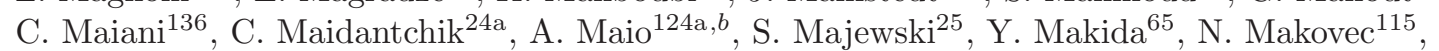

P. $\mathrm{Mal}^{136}$, B. Malaescu ${ }^{30}$, Pa. Malecki ${ }^{39}$, P. Malecki ${ }^{39}$, V.P. Maleev ${ }^{121}$, F. Malek ${ }^{55}$, U. Mallik ${ }^{62}$,

D. Malon ${ }^{6}$, C. Malone ${ }^{143}$, S. Maltezos ${ }^{10}$, V. Malyshev ${ }^{107}$, S. Malyukov ${ }^{30}$, R. Mameghani ${ }^{98}$,

J. Mamuzic ${ }^{13 b}$, A. Manabe ${ }^{65}$, L. Mandelli ${ }^{89 a}$, I. Mandić $^{74}$, R. Mandrysch ${ }^{16}$, J. Maneira ${ }^{124 a}$,

A. Manfredini ${ }^{99}$, L. Manhaes de Andrade Filho ${ }^{24 b}$, J.A. Manjarres Ramos ${ }^{136}$, A. Mann ${ }^{54}$,

P.M. Manning ${ }^{137}$, A. Manousakis-Katsikakis ${ }^{9}$, B. Mansoulie ${ }^{136}$, A. Mapelli ${ }^{30}$ L. Mapelli $^{30}$,

L. March ${ }^{167}$, J.F. Marchand ${ }^{29}$, F. Marchese ${ }^{133 a, 133 b}$, G. Marchiori ${ }^{78}$, M. Marcisovsky ${ }^{125}$,

C.P. Marino ${ }^{169}$, F. Marroquim ${ }^{24 a}$, Z. Marshall ${ }^{30}$, L.F. Marti ${ }^{17}$, S. Marti-Garcia ${ }^{167}$, B. Martin ${ }^{30}$,

B. Martin 88 , J.P. Martin 93 , T.A. Martin ${ }^{18}$, V.J. Martin ${ }^{46}$, B. Martin dit Latour ${ }^{49}$,

S. Martin-Haugh ${ }^{149}$, M. Martinez ${ }^{12}$, V. Martinez Outschoorn ${ }^{57}$, A.C. Martyniuk ${ }^{169}$, M. Marx ${ }^{82}$,

F. Marzano ${ }^{132 a}$, A. Marzin ${ }^{111}$, L. Masetti ${ }^{81}$, T. Mashimo ${ }^{155}$, R. Mashinistov ${ }^{94}$, J. Masik ${ }^{82}$,

A.L. Maslennikov ${ }^{107}$, I. Massa ${ }^{20 a, 20 b}$, G. Massaro $^{105}$, N. Massol ${ }^{5}$, P. Mastrandrea $^{148}$,

A. Mastroberardino ${ }^{37 a, 37 b}$, T. Masubuchi ${ }^{155}$, P. Matricon ${ }^{115}$, H. Matsunaga ${ }^{155}$, T. Matsushita ${ }^{66}$,

C. Mattravers ${ }^{118, c}$, J. Maurer ${ }^{83}$, S.J. Maxfield ${ }^{73}$, A. Mayne ${ }^{139}$, R. Mazini ${ }^{151}$, M. Mazur ${ }^{21}$,

L. Mazzaferro ${ }^{133 a, 133 b}$, M. Mazzanti ${ }^{89 a}$, J. Mc Donald ${ }^{85}$, S.P. Mc Kee ${ }^{87}$, A. McCarn ${ }^{165}$,

R.L. McCarthy ${ }^{148}$, T.G. McCarthy ${ }^{29}$, N.A. McCubbin ${ }^{129}$, K.W. McFarlane ${ }^{56, *}$, J.A. Mcfayden ${ }^{139}$,

G. Mchedlidze ${ }^{51 b}$, T. Mclaughlan ${ }^{18}$, S.J. McMahon ${ }^{129}$, R.A. McPherson ${ }^{169, k}$, A. Meade ${ }^{84}$,

J. Mechnich ${ }^{105}$, M. Mechtel ${ }^{175}$, M. Medinnis ${ }^{42}$, S. Meehan ${ }^{31}$, R. Meera-Lebbai ${ }^{111}$, T. Meguro ${ }^{116}$,

S. Mehlhase ${ }^{36}$, A. Mehta ${ }^{73}$, K. Meier ${ }^{58 a}$, B. Meirose ${ }^{79}$, C. Melachrinos ${ }^{31}$, B.R. Mellado Garcia ${ }^{173}$,

F. Meloni ${ }^{89 a, 89 b}$, L. Mendoza Navas ${ }^{162}$, Z. Meng ${ }^{151, w}$, A. Mengarelli20a,20b, S. Menke ${ }^{99}$,

E. Meoni ${ }^{161}$, K.M. Mercurio ${ }^{57}$, P. Mermod ${ }^{49}$, L. Merola ${ }^{102 a, 102 b}$, C. Meroni ${ }^{89 a}$, F.S. Merritt ${ }^{31}$,

H. Merritt ${ }^{109}$, A. Messina ${ }^{30, x}$, J. Metcalfe ${ }^{25}$, A.S. Mete ${ }^{163}$, C. Meyer ${ }^{81}$, C. Meyer ${ }^{31}$,

J-P. Meyer ${ }^{136}$, J. Meyer ${ }^{174}$, J. Meyer ${ }^{54}$, S. Michal ${ }^{30}$, L. Micu'26a, R.P. Middleton ${ }^{129}$, S. Migas ${ }^{73}$,

L. Mijović ${ }^{136}$, G. Mikenberg ${ }^{172}$, M. Mikestikova ${ }^{125}$, M. Mikuž ${ }^{74}$, D.W. Miller ${ }^{31}$, R.J. Miller ${ }^{88}$,

W.J. Mills ${ }^{168}$, C. Mills ${ }^{57}$, A. Milov ${ }^{172}$, D.A. Milstead ${ }^{146 a, 146 b}$, D. Milstein ${ }^{172}$, A.A. Minaenko ${ }^{128}$,

M. Miñano Moya ${ }^{167}$, I.A. Minashvili ${ }^{64}$, A.I. Mincer ${ }^{108}$, B. Mindur ${ }^{38}$, M. Mineev ${ }^{64}$, Y. Ming ${ }^{173}$,

L.M. Mir ${ }^{12}$, G. Mirabelli ${ }^{132 a}$, J. Mitrevski ${ }^{137}$, V.A. Mitsou ${ }^{167}$, S. Mitsui ${ }^{65}$, P.S. Miyagawa ${ }^{139}$,

J.U. Mjörnmark ${ }^{79}$, T. Moa ${ }^{146 a, 146 b}$, V. Moeller ${ }^{28}$, K. Mönig ${ }^{42}$, N. Möser ${ }^{21}$, S. Mohapatra ${ }^{148}$,

W. Mohr ${ }^{48}$, R. Moles-Valls ${ }^{167}$, A. Molfetas $^{30}$, J. Monk ${ }^{77}$, E. Monnier ${ }^{83}$, J. Montejo Berlingen ${ }^{12}$,

F. Monticelli ${ }^{70}$, S. Monzani20a,20b, R.W. Moore ${ }^{3}$, G.F. Moorhead ${ }^{86}$, C. Mora Herrera ${ }^{49}$,

A. Moraes ${ }^{53}$, N. Morange ${ }^{136}$, J. Morel ${ }^{54}$, G. Morello ${ }^{37 a, 37 b}$, D. Moreno ${ }^{81}$, M. Moreno Llácer ${ }^{167}$,

P. Morettini ${ }^{50 a}$, M. Morgenstern ${ }^{44}$, M. Morii ${ }^{57}$, A.K. Morley ${ }^{30}$, G. Mornacchi ${ }^{30}$, J.D. Morris ${ }^{75}$,

L. Morvaj ${ }^{101}$, H.G. Moser ${ }^{99}$, M. Mosidze ${ }^{51 b}$, J. Moss ${ }^{109}$, R. Mount ${ }^{143}$, E. Mountricha ${ }^{10, y}$,

S.V. Mouraviev ${ }^{94, *}$, E.J.W. Moyse ${ }^{84}$, F. Mueller ${ }^{58 a}$, J. Mueller ${ }^{123}$, K. Mueller ${ }^{21}$, T.A. Müller ${ }^{98}$,

T. Mueller ${ }^{81}$, D. Muenstermann ${ }^{30}$, Y. Munwes ${ }^{153}$, W.J. Murray ${ }^{129}$, I. Mussche ${ }^{105}$,

E. Musto ${ }^{102 a, 102 b}$, A.G. Myagkov ${ }^{128}$, M. Myska ${ }^{125}$, O. Nackenhorst ${ }^{54}$, J. Nadal ${ }^{12}$, K. Nagai ${ }^{160}$, 
R. Nagai ${ }^{157}$, K. Nagano ${ }^{65}$, A. Nagarkar ${ }^{109}$, Y. Nagasaka ${ }^{59}$, M. Nagel ${ }^{99}$, A.M. Nairz ${ }^{30}$, Y. Nakahama ${ }^{30}$, K. Nakamura ${ }^{155}$, T. Nakamura ${ }^{155}$, I. Nakano ${ }^{110}$, G. Nanava ${ }^{21}$, A. Napier ${ }^{161}$, R. Narayan ${ }^{58 b}$, M. Nash ${ }^{77, c}$, T. Nattermann ${ }^{21}$, T. Naumann ${ }^{42}$, G. Navarro ${ }^{162}$, H.A. Neal ${ }^{87}$, P.Yu. Nechaeva ${ }^{94}$, T.J. Neep ${ }^{82}$, A. Negri ${ }^{119 a, 119 b}$, G. Negri ${ }^{30}$, M. Negrini $^{20 a}$, S. Nektarijevic ${ }^{49}$, A. Nelson ${ }^{163}$, T.K. Nelson ${ }^{143}$, S. Nemecek ${ }^{125}$, P. Nemethy ${ }^{108}$, A.A. Nepomuceno ${ }^{24 a}$, M. Nessi ${ }^{30, z}$, M.S. Neubauer ${ }^{165}$, M. Neumann ${ }^{175}$, A. Neusiedl ${ }^{81}$, R.M. Neves ${ }^{108}$, P. Nevski ${ }^{25}$, F.M. Newcomer ${ }^{120}$, P.R. Newman ${ }^{18}$, V. Nguyen Thi Hong ${ }^{136}$, R.B. Nickerson ${ }^{118}$,

R. Nicolaidou ${ }^{136}$, B. Nicquevert ${ }^{30}$, F. Niedercorn ${ }^{115}$, J. Nielsen ${ }^{137}$, N. Nikiforou ${ }^{35}$, A. Nikiforov ${ }^{16}$, V. Nikolaenko ${ }^{128}$, I. Nikolic-Audit ${ }^{78}$, K. Nikolics ${ }^{49}$, K. Nikolopoulos ${ }^{18}$, H. Nilsen ${ }^{48}$, P. Nilsson ${ }^{8}$, Y. Ninomiya ${ }^{155}$, A. Nisati ${ }^{132 a}$, R. Nisius ${ }^{99}$, T. Nobe ${ }^{157}$, L. Nodulman $^{6}$, M. Nomachi ${ }^{116}$, I. Nomidis ${ }^{154}$, S. Norberg ${ }^{111}$, M. Nordberg ${ }^{30}$, P.R. Norton ${ }^{129}$, J. Novakova ${ }^{126}$, M. Nozaki ${ }^{65}$, L. Nozka ${ }^{113}$, I.M. Nugent ${ }^{159 a}$, A.-E. Nuncio-Quiroz ${ }^{21}$, G. Nunes Hanninger ${ }^{86}$, T. Nunnemann ${ }^{98}$, E. Nurse ${ }^{77}$, B.J. O'Brien ${ }^{46}$, D.C. O'Neil ${ }^{142}$, V. O'Shea ${ }^{53}$, L.B. Oakes ${ }^{98}$, F.G. Oakham ${ }^{29, d}$, H. Oberlack ${ }^{99}$, J. Ocariz ${ }^{78}$, A. Ochi ${ }^{66}$, S. Oda ${ }^{69}$, S. Odaka ${ }^{65}$, J. Odier ${ }^{83}$, H. Ogren ${ }^{60}$, A. Oh $^{82}$, S.H. $\mathrm{Oh}^{45}$, C.C. $\mathrm{Ohm}^{30}$, T. Ohshima ${ }^{101}$, W. Okamura ${ }^{116}$, H. Okawa ${ }^{25}$, Y. Okumura ${ }^{31}$, T. Okuyama ${ }^{155}$, A. Olariu ${ }^{26 a}$, A.G. Olchevski64, S.A. Olivares Pino ${ }^{32 a}$, M. Oliveira ${ }^{124 a, h}$, D. Oliveira Damazio ${ }^{25}$, E. Oliver Garcia ${ }^{167}$, D. Olivito ${ }^{120}$, A. Olszewski ${ }^{39}$, J. Olszowska ${ }^{39}$, A. Onofre ${ }^{124 a, a a}$, P.U.E. Onyisi ${ }^{31}$, C.J. Oram ${ }^{159 a}$, M.J. Oreglia ${ }^{31}$, Y. Oren ${ }^{153}$,

D. Orestano ${ }^{134 a, 134 b}$, N. Orlando ${ }^{72 a, 72 b}$, I. Orlov ${ }^{107}$, C. Oropeza Barrera ${ }^{53}$, R.S. Orr ${ }^{158}$,

B. Osculati ${ }^{50 a, 50 b}$, R. Ospanov ${ }^{120}$, C. Osuna ${ }^{12}$, G. Otero y Garzon ${ }^{27}$, J.P. Ottersbach ${ }^{105}$, M. Ouchrif ${ }^{135 d}$, E.A. Ouellette ${ }^{169}$, F. Ould-Saada ${ }^{117}$, A. Ouraou ${ }^{136}$, Q. Ouyang ${ }^{33 a}$,

A. Ovcharova ${ }^{15}$, M. Owen ${ }^{82}$, S. Owen ${ }^{139}$, V.E. Ozcan ${ }^{19 a}$, N. Ozturk ${ }^{8}$, A. Pacheco Pages ${ }^{12}$, C. Padilla Aranda ${ }^{12}$, S. Pagan Griso ${ }^{15}$, E. Paganis ${ }^{139}$, C. Pahl ${ }^{99}$, F. Paige ${ }^{25}$, P. Pais ${ }^{84}$, K. Pajchel ${ }^{117}$, G. Palacino ${ }^{159 b}$, C.P. Paleari ${ }^{7}$, S. Palestini ${ }^{30}$, D. Pallin ${ }^{34}$, A. Palma ${ }^{124 a}$, J.D. Palmer ${ }^{18}$, Y.B. Pan $^{173}$, E. Panagiotopoulou ${ }^{10}$, J.G. Panduro Vazquez ${ }^{76}$, P. Pani ${ }^{105}$ N. Panikashvilii ${ }^{87}$, S. Panitkin ${ }^{25}$, D. Pantea ${ }^{26 a}$, A. Papadelis ${ }^{146 a}$, Th.D. Papadopoulou ${ }^{10}$, A. Paramonov ${ }^{6}$, D. Paredes Hernandez ${ }^{34}$, W. Park ${ }^{25, a b}$, M.A. Parker ${ }^{28}$, F. Parodi ${ }^{50 a, 50 b}$, J.A. Parsons ${ }^{35}$, U. Parzefall ${ }^{48}$, S. Pashapour ${ }^{54}$, E. Pasqualucci ${ }^{132 a}$, S. Passaggio ${ }^{50 a}$, A. Passeri ${ }^{134 a}$, F. Pastore $134 a, 134 b, *, F r . P^{1}$ astore ${ }^{76}$, G. Pásztor ${ }^{49, a c}$, S. Pataraia ${ }^{175}$, N. Patel ${ }^{150}$, J.R. Pater ${ }^{82}$, S. Patricelli102a,102b, T. Pauly ${ }^{30}$, M. Pecsy ${ }^{144 a}$, S. Pedraza Lopez ${ }^{167}$, M.I. Pedraza Morales ${ }^{173}$, S.V. Peleganchuk ${ }^{107}$, D. Pelikan ${ }^{166}$, H. Peng ${ }^{33 b}$, B. Penning ${ }^{31}$, A. Penson ${ }^{35}$, J. Penwell ${ }^{60}$, M. Perantoni ${ }^{24 a}$, K. Perez ${ }^{35, a d}$, T. Perez Cavalcanti ${ }^{42}$, E. Perez Codina ${ }^{159 a}$,

M.T. Pérez García-Estañ ${ }^{167}$, V. Perez Reale ${ }^{35}$, L. Perini ${ }^{89 a, 89 b}$, H. Pernegger ${ }^{30}$, R. Perrino ${ }^{72 a}$, P. Perrodo ${ }^{5}$, V.D. Peshekhonov ${ }^{64}$, K. Peters ${ }^{30}$, B.A. Petersen ${ }^{30}$, J. Petersen ${ }^{30}$, T.C. Petersen ${ }^{36}$, E. Petit ${ }^{5}$, A. Petridis ${ }^{154}$, C. Petridou ${ }^{154}$, E. Petrolo132a, F. Petrucci ${ }^{134 a, 134 b}$, D. Petschull ${ }^{42}$, M. Petteni ${ }^{142}$, R. Pezoa ${ }^{32 b}$, A. Phan ${ }^{86}$, P.W. Phillips ${ }^{129}$, G. Piacquadio ${ }^{30}$, A. Picazio ${ }^{49}$, E. Piccaro ${ }^{75}$, M. Piccinini ${ }^{20 a, 20 b}$, S.M. Piec ${ }^{42}$, R. Piegaia ${ }^{27}$, D.T. Pignotti ${ }^{109}$, J.E. Pilcher ${ }^{31}$, A.D. Pilkington ${ }^{82}$, J. Pina ${ }^{124 a, b}$, M. Pinamonti ${ }^{164 a, 164 c}$, A. Pinder ${ }^{118}$, J.L. Pinfold ${ }^{3}$, B. Pinto ${ }^{124 a}$, C. Pizio ${ }^{89 a}, 89 b$, M. Plamondon ${ }^{169}$, M.-A. Pleier ${ }^{25}$, E. Plotnikova ${ }^{64}$, A. Poblaguev ${ }^{25}$, S. Poddar ${ }^{58 a}$,

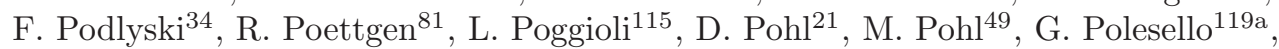

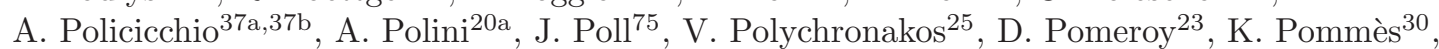
L. Pontecorvo ${ }^{132 a}$, B.G. Pope ${ }^{88}$, G.A. Popeneciu ${ }^{26 a}$, D.S. Popovic ${ }^{13 a}$, A. Poppleton ${ }^{30}$, X. Portell Bueso ${ }^{30}$, G.E. Pospelov ${ }^{99}$, S. Pospisil ${ }^{127}$, I.N. Potrap ${ }^{99}$, C.J. Potter ${ }^{149}$, C.T. Potter ${ }^{114}$, G. Poulard ${ }^{30}$, J. Poveda ${ }^{60}$, V. Pozdnyakov ${ }^{64}$, R. Prabhu ${ }^{77}$, P. Pralavorio ${ }^{83}$, A. Pranko ${ }^{15}$,

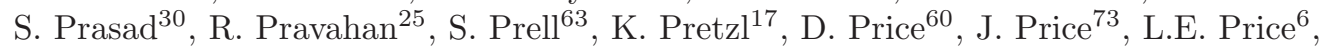
D. Prieur ${ }^{123}$, M. Primavera ${ }^{72 a}$, K. Prokofiev ${ }^{108}$, F. Prokoshin ${ }^{32 b}$, S. Protopopescu ${ }^{25}$,

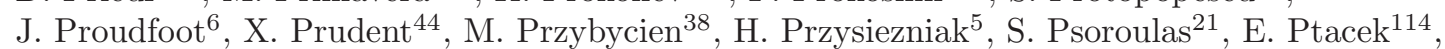
E. Pueschel ${ }^{84}$, J. Purdham ${ }^{87}$, M. Purohit ${ }^{25, a b}$, P. Puzo ${ }^{115}$, Y. Pylypchenko ${ }^{62}$, J. Qian ${ }^{87}$, A. Quadt ${ }^{54}$, D.R. Quarrie ${ }^{15}$, W.B. Quayle ${ }^{173}$, F. Quinonez ${ }^{32 a}$, M. Raas ${ }^{104}$, V. Radeka ${ }^{25}$, V. Radescu ${ }^{42}$, P. Radloff ${ }^{114}$, F. Ragusa ${ }^{89 a, 89 b}$, G. Rahal ${ }^{178}$, A.M. Rahimi ${ }^{109}$, D. Rahm ${ }^{25}$, S. Rajagopalan ${ }^{25}$, M. Rammensee ${ }^{48}$, M. Rammes ${ }^{141}$, A.S. Randle-Conde ${ }^{40}$, K. Randrianarivony ${ }^{29}$, F. Rauscher ${ }^{98}$, T.C. Rave ${ }^{48}$, M. Raymond ${ }^{30}$, A.L. Read ${ }^{117}$, D.M. Rebuzzi ${ }^{119 a, 119 b}$, 
A. Redelbach ${ }^{174}$, G. Redlinger ${ }^{25}$, R. Reece ${ }^{120}$, K. Reeves ${ }^{41}$, A. Reinsch $^{114}$, I. Reisinger ${ }^{43}$, C. Rembser ${ }^{30}$, Z.L. Ren ${ }^{151}$, A. Renaud ${ }^{115}$, M. Rescigno ${ }^{132 a}$, S. Resconi ${ }^{89 a}$, B. Resende ${ }^{136}$, P. Reznicek ${ }^{98}$, R. Rezvani ${ }^{158}$, R. Richter ${ }^{99}$, E. Richter-Was ${ }^{5, a e}$, M. Ridel ${ }^{78}$, M. Rijpstra ${ }^{105}$, M. Rijssenbeek ${ }^{148}$, A. Rimoldi ${ }^{119 a, 119 b}$, L. Rinaldi ${ }^{20 a}$, R.R. Rios ${ }^{40}$, I. Riu ${ }^{12}$, G. Rivoltella ${ }^{89 a, 89 b}$ F. Rizatdinova ${ }^{112}$, E. Rizvi ${ }^{75}$, S.H. Robertson ${ }^{85, k}$, A. Robichaud-Veronneau ${ }^{118}$, D. Robinson ${ }^{28}$, J.E.M. Robinson ${ }^{82}$, A. Robson ${ }^{53}$, J.G. Rocha de Lima ${ }^{106}$, C. Roda ${ }^{122 a, 122 b}$,

D. Roda Dos Santos ${ }^{30}$, A. Roe ${ }^{54}$, S. Roe ${ }^{30}$, O. Røhne ${ }^{117}$, S. Rolli ${ }^{161}$, A. Romaniouk ${ }^{96}$,

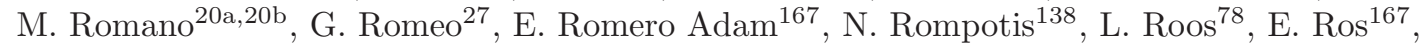
S. Rosati ${ }^{132 a}$, K. Rosbach ${ }^{49}$, A. Rose ${ }^{149}$, M. Rose ${ }^{76}$, G.A. Rosenbaum ${ }^{158}$, E.I. Rosenberg ${ }^{63}$, P.L. Rosendahl ${ }^{14}$, O. Rosenthal ${ }^{141}$, L. Rosselet ${ }^{49}$, V. Rossetti ${ }^{12}$, E. Rossi ${ }^{132 a, 132 b}$, L.P. Rossi ${ }^{50 a}$, M. Rotaru ${ }^{26 a}$, I. Roth ${ }^{172}$, J. Rothberg ${ }^{138}$, D. Rousseau ${ }^{115}$, C.R. Royon ${ }^{136}$, A. Rozanov ${ }^{83}$, Y. Rozen ${ }^{152}$, X. Ruan ${ }^{33 a, a f}$, F. Rubbo ${ }^{12}$, I. Rubinskiy ${ }^{42}$, N. Ruckstuhl ${ }^{105}$, V.I. Rud ${ }^{97}$, C. Rudolph ${ }^{44}$, G. Rudolph ${ }^{61}$, F. Rühr 7 , A. Ruiz-Martinez ${ }^{63}$, L. Rumyantsev ${ }^{64}$, Z. Rurikova ${ }^{48}$, N.A. Rusakovich ${ }^{64}$, A. Ruschke ${ }^{98}$, J.P. Rutherfoord ${ }^{7}$, P. Ruzicka ${ }^{125}$, Y.F. Ryabov ${ }^{121}$, M. Rybar ${ }^{126}$, G. Rybkin ${ }^{115}$, N.C. Ryder ${ }^{118}$, A.F. Saavedra ${ }^{150}$, I. Sadeh ${ }^{153}$, H.F-W. Sadrozinski ${ }^{137}$, R. Sadykov ${ }^{64}$, F. Safai Tehrani132a , H. Sakamoto ${ }^{155}$, G. Salamanna ${ }^{75}$, A. Salamon ${ }^{133 a}$, M. Saleem ${ }^{111}$, D. Salek ${ }^{30}$, D. Salihagic ${ }^{99}$, A. Salnikov ${ }^{143}$, J. Salt ${ }^{167}$, B.M. Salvachua Ferrando ${ }^{6}$, D. Salvatore ${ }^{37 a, 37 b}$, F. Salvatore ${ }^{149}$, A. Salvucci ${ }^{104}$, A. Salzburger ${ }^{30}$, D. Sampsonidis ${ }^{154}$,

B.H. Samset ${ }^{117}$, A. Sanchez ${ }^{102 a, 102 b}$, V. Sanchez Martinez ${ }^{167}$, H. Sandaker ${ }^{14}$, H.G. Sander ${ }^{81}$, M.P. Sanders ${ }^{98}$, M. Sandhoff ${ }^{175}$, T. Sandoval ${ }^{28}$, C. Sandoval ${ }^{162}$, R. Sandstroem ${ }^{99}$,

D.P.C. Sankey ${ }^{129}$, A. Sansoni ${ }^{47}$, C. Santamarina Rios ${ }^{85}$, C. Santoni ${ }^{34}$, R. Santonico ${ }^{133 a, 133 b}$, H. Santos ${ }^{124 a}$, I. Santoyo Castillo ${ }^{149}$, J.G. Saraiva ${ }^{124 a}$, T. Sarangi ${ }^{173}$, E. Sarkisyan-Grinbaum ${ }^{8}$, F. Sarri ${ }^{122 a, 122 b}$, G. Sartisohn ${ }^{175}$, O. Sasaki ${ }^{65}$, Y. Sasaki ${ }^{155}$, N. Sasao ${ }^{67}$, I. Satsounkevitch ${ }^{90}$, G. Sauvage ${ }^{5, *}$, E. Sauvan ${ }^{5}$, J.B. Sauvan ${ }^{115}$, P. Savard ${ }^{158, d}$, V. Savinov ${ }^{123}$, D.O. Savu ${ }^{30}$, L. Sawyer ${ }^{25, m}$, D.H. Saxon ${ }^{53}$, J. Saxon ${ }^{120}$, C. Sbarra ${ }^{20 a}$, A. Sbrizzi ${ }^{20 a, 20 b}$, D.A. Scannicchio ${ }^{163}$, M. Scarcella ${ }^{150}$, J. Schaarschmidt ${ }^{115}$, P. Schacht ${ }^{99}$, D. Schaefer ${ }^{120}$, U. Schäfer ${ }^{81}$, A. Schaelicke ${ }^{46}$, S. Schaepe ${ }^{21}$, S. Schaetzel ${ }^{58 b}$, A.C. Schaffer ${ }^{115}$, D. Schaile ${ }^{98}$, R.D. Schamberger ${ }^{148}$, A.G. Schamov ${ }^{107}$, V. Scharf 58 a, V.A. Schegelsky ${ }^{121}$, D. Scheirich ${ }^{87}$, M. Schernau ${ }^{163}$, M.I. Scherzer ${ }^{35}$, C. Schiavi50a,50b , J. Schieck ${ }^{98}$, M. Schioppa ${ }^{37 a, 37 b}$, S. Schlenker ${ }^{30}$, E. Schmidt ${ }^{48}$, K. Schmieden ${ }^{21}$, C. Schmitt ${ }^{81}$, S. Schmitt ${ }^{58 b}$, B. Schneider ${ }^{17}$, U. Schnoor ${ }^{44}$, L. Schoeffel ${ }^{136}$, A. Schoening ${ }^{58 b}$, A.L.S. Schorlemmer ${ }^{54}$, M. Schott ${ }^{30}$, D. Schouten ${ }^{159 a}$, J. Schovancova ${ }^{125}$, M. Schram ${ }^{85}$, C. Schroeder ${ }^{81}$, N. Schroer ${ }^{58 c}$, M.J. Schultens ${ }^{21}$, J. Schultes ${ }^{175}$,

H.-C. Schultz-Coulon ${ }^{58 a}$, H. Schulz ${ }^{16}$, M. Schumacher ${ }^{48}$, B.A. Schumm ${ }^{137}$, Ph. Schune ${ }^{136}$,

C. Schwanenberger ${ }^{82}$, A. Schwartzman ${ }^{143}$, Ph. Schwegler ${ }^{99}$, Ph. Schwemling ${ }^{78}$, R. Schwienhorst ${ }^{88}$, R. Schwierz ${ }^{44}$, J. Schwindling ${ }^{136}$, T. Schwindt ${ }^{21}$, M. Schwoerer ${ }^{5}$, F.G. Sciacca ${ }^{17}$, G. Sciolla ${ }^{23}$, W.G. Scott ${ }^{129}$, J. Searcy ${ }^{114}$, G. Sedov ${ }^{42}$, E. Sedykh ${ }^{121}$, S.C. Seidel ${ }^{103}$, A. Seiden ${ }^{137}$, F. Seifert ${ }^{44}$, J.M. Seixas ${ }^{24 a}$, G. Sekhniaidze ${ }^{102 a}$, S.J. Sekula ${ }^{40}$, K.E. Selbach ${ }^{46}$, D.M. Seliverstov ${ }^{121}$,

B. Sellden ${ }^{146 a}$, G. Sellers ${ }^{73}$, M. Seman ${ }^{144 b}$, N. Semprini-Cesari20a,20b, C. Serfon ${ }^{98}$, L. Serin ${ }^{115}$, L. Serkin ${ }^{54}$, R. Seuster ${ }^{159 a}$, H. Severini ${ }^{111}$, A. Sfyrla ${ }^{30}$, E. Shabalina ${ }^{54}$, M. Shamim ${ }^{114}$, L.Y. Shan ${ }^{33 a}$, J.T. Shank ${ }^{22}$, Q.T. Shao ${ }^{86}$, M. Shapiro ${ }^{15}$, P.B. Shatalov ${ }^{95}$, K. Shaw ${ }^{164 a, 164 c}$, D. Sherman ${ }^{176}$, P. Sherwood ${ }^{77}$, S. Shimizu ${ }^{101}$, M. Shimojima ${ }^{100}$, T. Shin ${ }^{56}$, M. Shiyakova ${ }^{64}$, A. Shmeleva ${ }^{94}$, M.J. Shochet ${ }^{31}$, D. Short ${ }^{118}$, S. Shrestha ${ }^{63}$, E. Shulga ${ }^{96}$, M.A. Shupe ${ }^{7}$, P. Sicho ${ }^{125}$, A. Sidoti ${ }^{132 a}$, F. Siegert ${ }^{48}$, Dj. Sijacki ${ }^{13 a}$, O. Silbert ${ }^{172}$, J. Silva ${ }^{124 a}$, Y. Silver ${ }^{153}$, D. Silverstein ${ }^{143}$, S.B. Silverstein ${ }^{146 a}$, V. Simak ${ }^{127}$, O. Simard ${ }^{136}$, Lj. Simic ${ }^{13 a}$, S. Simion ${ }^{115}$, E. Simioni ${ }^{81}$, B. Simmons ${ }^{77}$, R. Simoniello ${ }^{89 a, 89 b}$, M. $\operatorname{Simonyan}^{36}$, P. Sinervo ${ }^{158}$, N.B. Sinev ${ }^{114}$, V. Sipica ${ }^{141}$, G. Siragusa ${ }^{174}$, A. Sircar ${ }^{25}$, A.N. Sisakyan ${ }^{64, *}$, S.Yu. Sivoklokov ${ }^{97}$, J. Sjölin ${ }^{146 a, 146 b}$, T.B. Sjursen ${ }^{14}$, L.A. Skinnari ${ }^{15}$, H.P. Skottowe ${ }^{57}$, K. Skovpen ${ }^{107}$, P. Skubic ${ }^{111}$, M. Slater ${ }^{18}$ T. Slavicek ${ }^{127}$, K. Sliwa ${ }^{161}$, V. Smakhtin ${ }^{172}$, B.H. Smart ${ }^{46}$, L. Smestad ${ }^{117}$, S.Yu. Smirnov ${ }^{96}$, Y. Smirnov ${ }^{96}$, L.N. Smirnova ${ }^{97}$, O. Smirnova ${ }^{79}$, B.C. Smith ${ }^{57}$, D. Smith ${ }^{143}$, K.M. Smith ${ }^{53}$, M. Smizanska ${ }^{71}$, K. Smolek ${ }^{127}$, A.A. Snesarev ${ }^{94}$, S.W. Snow ${ }^{82}$, J. Snow ${ }^{111}$, S. Snyder ${ }^{25}$, R. Sobie ${ }^{169, k}$, J. Sodomka ${ }^{127}$, A. Soffer ${ }^{153}$, C.A. Solans ${ }^{167}$, M. Solar ${ }^{127}$, J. Solc ${ }^{127}$, E.Yu. Soldatov ${ }^{96}$, U. Soldevila ${ }^{167}$, E. Solfaroli Camillocci132a,132b, A.A. Solodkov ${ }^{128}$, 
O.V. Solovyanov ${ }^{128}$, V. Solovyev ${ }^{121}$, N. Soni ${ }^{1}$, V. Sopko ${ }^{127}$, B. Sopko ${ }^{127}$, M. Sosebee ${ }^{8}$, R. Soualah ${ }^{164 a, 164 c}$, A. Soukharev ${ }^{107}$, S. Spagnolo ${ }^{72 a, 72 b}$, F. Spanò ${ }^{76}$, R. Spighi ${ }^{20 a}$, G. Spigo ${ }^{30}$, R. Spiwoks $^{30}$, M. Spousta ${ }^{126, a g}$, T. Spreitzer ${ }^{158}$, B. Spurlock ${ }^{8}$, R.D. St. Denis ${ }^{53}$, J. Stahlman ${ }^{120}$, R. Stamen ${ }^{58 a}$, E. Stanecka ${ }^{39}$, R.W. Stanek ${ }^{6}$, C. Stanescu ${ }^{134 a}$, M. Stanescu-Bellu ${ }^{42}$, M.M. Stanitzki ${ }^{42}$, S. Stapnes ${ }^{117}$, E.A. Starchenko ${ }^{128}$, J. Stark ${ }^{55}$, P. Staroba ${ }^{125}$, P. Starovoitov ${ }^{42}$, R. Staszewski ${ }^{39}$, A. Staude ${ }^{98}$, P. Stavina ${ }^{144 a, *}$, G. Steele ${ }^{53}$, P. Steinbach ${ }^{44}$, P. Steinberg ${ }^{25}$, I. Stekl ${ }^{127}$, B. Stelzer ${ }^{142}$, H.J. Stelzer ${ }^{88}$, O. Stelzer-Chilton ${ }^{159 a}$, H. Stenzel ${ }^{52}$, S. Stern ${ }^{99}$, G.A. Stewart ${ }^{30}$, J.A. Stillings ${ }^{21}$, M.C. Stockton ${ }^{85}$, K. Stoerig ${ }^{48}$, G. Stoicea ${ }^{26 a}$, S. Stonjek ${ }^{99}$, P. Strachota ${ }^{126}$, A.R. Stradling ${ }^{8}$, A. Straessner ${ }^{44}$, J. Strandberg ${ }^{147}$, S. Strandberg ${ }^{146 a, 146 b}$, A. Strandlie ${ }^{117}$, M. Strang ${ }^{109}$, E. Strauss ${ }^{143}$, M. Strauss ${ }^{111}$, P. Strizenec ${ }^{144 b}$, R. Ströhmer ${ }^{174}$, D.M. Strom ${ }^{114}$, J.A. Strong ${ }^{76, *}$, R. Stroynowski ${ }^{40}$, B. Stugu ${ }^{14}$, I. Stumer ${ }^{25, *}$, J. Stupak ${ }^{148}$, P. Sturm ${ }^{175}$, N.A. Styles ${ }^{42}$, D.A. Soh ${ }^{151, u}$, D. Su ${ }^{143}$, HS. Subramania ${ }^{3}$, R. Subramaniam ${ }^{25}$, A. Succurro ${ }^{12}$, Y. Sugaya ${ }^{116}$, C. Suhr ${ }^{106}$, M. Suk ${ }^{126}$, V.V. Sulin ${ }^{94}$, S. Sultansoy ${ }^{4 d}$, T. Sumida ${ }^{67}$, X. Sun $^{55}$, J.E. Sundermann ${ }^{48}$, K. Suruliz ${ }^{139}$, G. Susinno $37 a, 37 b$, M.R. Sutton ${ }^{149}$, Y. Suzuki ${ }^{65}$, Y. Suzuki ${ }^{66}$, M. Svatos ${ }^{125}$, S. Swedish ${ }^{168}$, I. Sykora ${ }^{144 a}$, T. Sykora ${ }^{126}$, J. Sánchez ${ }^{167}$, D. Ta ${ }^{105}$, K. Tackmann ${ }^{42}$, A. Taffard ${ }^{163}$, R. Tafirout ${ }^{159 a}$, N. Taiblum ${ }^{153}$, Y. Takahashi ${ }^{101}$, H. Takai ${ }^{25}$, R. Takashima ${ }^{68}$, H. Takeda ${ }^{66}$, T. Takeshita ${ }^{140}$, Y. Takubo ${ }^{65}$, M. Talby $^{83}$, A. Talyshev ${ }^{107, f}$, M.C. Tamsett ${ }^{25}$, K.G. Tan $^{86}$, J. Tanaka ${ }^{155}$, R. Tanaka ${ }^{115}$, S. Tanaka ${ }^{131}$, S. Tanaka ${ }^{65}$, A.J. Tanasijczuk ${ }^{142}$, K. Tani ${ }^{66}$, N. Tannoury ${ }^{83}$, S. Tapprogge ${ }^{81}$, D. Tardif ${ }^{158}$, S. Tarem ${ }^{152}$, F. Tarrade ${ }^{29}$, G.F. Tartarelli ${ }^{89 a}$, P. Tas ${ }^{126}$, M. Tasevsky ${ }^{125}$, E. Tassi ${ }^{37 a, 37 b}$, Y. Tayalati ${ }^{135 d}$ C. Taylor ${ }^{77}$, F.E. Taylor ${ }^{92}$, G.N. Taylor ${ }^{86}$, W. Taylor ${ }^{159 b}$, M. Teinturier ${ }^{115}$, F.A. Teischinger ${ }^{30}$, M. Teixeira Dias Castanheira ${ }^{75}$, P. Teixeira-Dias ${ }^{76}$, K.K. Temming ${ }^{48}$, H. Ten Kate ${ }^{30}$, P.K. Teng ${ }^{151}$, S. Terada ${ }^{65}$, K. Terashi ${ }^{155}$, J. Terron ${ }^{80}$, M. Testa ${ }^{47}$, R.J. Teuscher ${ }^{158, k}$, J. Therhaag ${ }^{21}$, T. Theveneaux-Pelzer ${ }^{78}$, S. Thoma ${ }^{48}$, J.P. Thomas ${ }^{18}$, E.N. Thompson ${ }^{35}$, P.D. Thompson ${ }^{18}$, P.D. Thompson ${ }^{158}$, A.S. Thompson ${ }^{53}$, L.A. Thomsen ${ }^{36}$, E. Thomson ${ }^{120}$, M. Thomson ${ }^{28}$, W.M. Thong ${ }^{86}$, R.P. Thun ${ }^{87}$, F. $\operatorname{Tian}^{35}$, M.J. Tibbetts ${ }^{15}$, T. Tic ${ }^{125}$, V.O. Tikhomirov ${ }^{94}$, Y.A. Tikhonov ${ }^{107, f}$, S. Timoshenko ${ }^{96}$, E. Tiouchichine ${ }^{83}$, P. Tipton ${ }^{176}$, S. Tisserant ${ }^{83}$, T. Todorov ${ }^{5}$, S. Todorova-Nova ${ }^{161}$, B. Toggerson ${ }^{163}$, J. Tojo ${ }^{69}$, S. Tokár ${ }^{144 a}$,

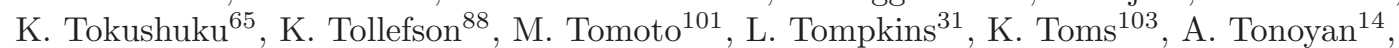
C. Topfel ${ }^{17}$, N.D. Topilin ${ }^{64}$, E. Torrence ${ }^{114}$, H. Torres ${ }^{78}$, E. Torró Pastor ${ }^{167}$, J. Toth ${ }^{83, a c}$,

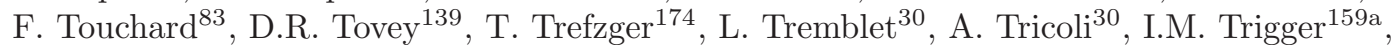
S. Trincaz-Duvoid ${ }^{78}$, M.F. Tripiana ${ }^{70}$, N. Triplett ${ }^{25}$, W. Trischuk ${ }^{158}$, B. Trocmé ${ }^{55}$, C. Troncon ${ }^{89 a}$, M. Trottier-McDonald ${ }^{142}$, P. True ${ }^{88}$, M. Trzebinski ${ }^{39}$, A. Trzupek ${ }^{39}$, C. Tsarouchas ${ }^{30}$, J.C-L. Tseng ${ }^{118}$, M. Tsiakiris ${ }^{105}$, P.V. Tsiareshka ${ }^{90}$, D. Tsionou ${ }^{5, a h}$, G. Tsipolitis ${ }^{10}$, S. Tsiskaridze ${ }^{12}$, V. Tsiskaridze ${ }^{48}$, E.G. Tskhadadze ${ }^{51 a}$, I.I. Tsukerman ${ }^{95}$, V. Tsulaia ${ }^{15}$, J.-W. Tsung ${ }^{21}$, S. Tsuno ${ }^{65}$, D. Tsybychev ${ }^{148}$, A. Tua ${ }^{139}$, A. Tudorache ${ }^{26 a}$, V. Tudorache ${ }^{26 a}$, J.M. Tuggle ${ }^{31}$, M. Turala ${ }^{39}$, D. Turecek ${ }^{127}$, I. Turk Cakir ${ }^{4 e}$, E. Turlay ${ }^{105}$, R. Turra ${ }^{89 a, 89 b}$, P.M. Tuts ${ }^{35}$, A. Tykhonov ${ }^{74}$, M. Tylmad ${ }^{146 a, 146 b}$, M. Tyndel ${ }^{129}$, G. Tzanakos ${ }^{9}$, K. Uchida ${ }^{21}$, I. Ueda ${ }^{155}$, R. Ueno ${ }^{29}$, M. Ugland ${ }^{14}$, M. Uhlenbrock ${ }^{21}$, M. Uhrmacher ${ }^{54}$, F. Ukegawa ${ }^{160}$,

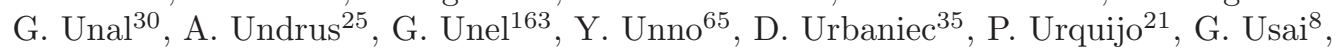
M. Uslenghi ${ }^{119 a, 119 b}$, L. Vacavant ${ }^{83}$, V. Vacek ${ }^{127}$, B. Vachon ${ }^{85}$, S. Vahsen ${ }^{15}$, J. Valenta ${ }^{125}$,

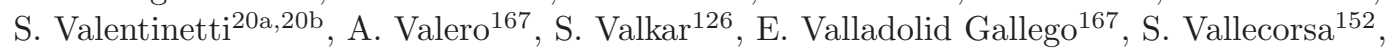
J.A. Valls Ferrer ${ }^{167}$, R. Van Berg ${ }^{120}$, P.C. Van Der Deijl ${ }^{105}$, R. van der Geer ${ }^{105}$,

H. van der Graaf ${ }^{105}$, R. Van Der Leeuw ${ }^{105}$, E. van der Poel ${ }^{105}$, D. van der Ster ${ }^{30}$, N. van Eldik ${ }^{30}$, P. van Gemmeren ${ }^{6}$, I. van Vulpen ${ }^{105}$, M. Vanadia ${ }^{99}$, W. Vandelli ${ }^{30}$, A. Vaniachine ${ }^{6}$, P. Vankov ${ }^{42}$, F. Vannucci ${ }^{78}$, R. Vari ${ }^{132 a}$, T. Varol $^{84}$, D. $\operatorname{Varouchas}^{15}$, A. $\operatorname{Vartapetian}^{8}$, K.E. $\operatorname{Varvell}^{150}$, V.I. Vassilakopoulos ${ }^{56}$, F. Vazeille ${ }^{34}$, T. Vazquez Schroeder ${ }^{54}$, G. Vegni ${ }^{89 a, 89 b}$, J.J. Veillet ${ }^{115}$,

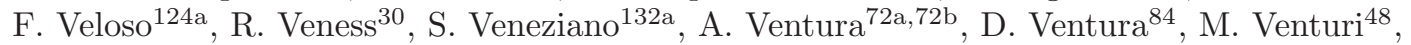
N. Venturi ${ }^{158}$, V. Vercesi ${ }^{119 a}$, M. Verducci ${ }^{138}$, W. Verkerke ${ }^{105}$, J.C. Vermeulen ${ }^{105}$, A. Vest ${ }^{44}$, M.C. Vetterli ${ }^{142, d}$, I. Vichou ${ }^{165}$, T. Vickey ${ }^{145 b, a i}$, O.E. Vickey Boeriu ${ }^{145 b}$, G.H.A. Viehhauser ${ }^{118}$, S. Viel ${ }^{168}$, M. Villa ${ }^{20 a, 20 b}$, M. Villaplana Perez ${ }^{167}$, E. Vilucchi ${ }^{47}$, M.G. Vincter ${ }^{29}$, E. Vinek ${ }^{30}$, V.B. Vinogradov ${ }^{64}$, M. Virchaux ${ }^{136, *}$,J. Virzi ${ }^{15}$, O. Vitells ${ }^{172}$, M. Viti ${ }^{42}$, I. Vivarelli ${ }^{48}$, 


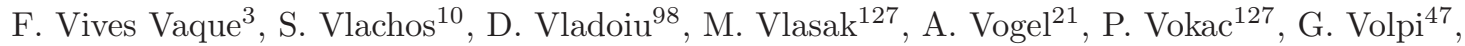
M. Volpi ${ }^{86}$, G. Volpini ${ }^{89 a}$, H. von der Schmitt ${ }^{99}$, H. von Radziewski ${ }^{48}$, E. von Toerne ${ }^{21}$,

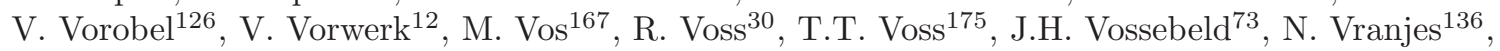
M. Vranjes Milosavljevic ${ }^{105}$, V. Vrba ${ }^{125}$, M. Vreeswijk ${ }^{105}$, T. Vu Anh ${ }^{48}$, R. Vuillermet $^{30}$, I. Vukotic ${ }^{31}$, W. Wagner ${ }^{175}$, P. Wagner ${ }^{120}$, H. Wahlen ${ }^{175}$, S. Wahrmund ${ }^{44}$, J. Wakabayashi ${ }^{101}$, S. Walch ${ }^{87}$, J. Walder ${ }^{71}$, R. Walker ${ }^{98}$, W. Walkowiak ${ }^{141}$, R. Wall ${ }^{176}$, P. Waller ${ }^{73}$, B. Walsh ${ }^{176}$, C. Wang ${ }^{45}$, H. Wang ${ }^{173}$, H. Wang ${ }^{33 \mathrm{~b}, a j}$, J. Wang ${ }^{151}$, J. Wang ${ }^{55}$, R. Wang ${ }^{103}$, S.M. Wang ${ }^{151}$,

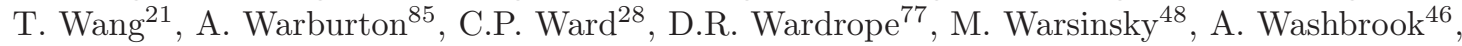

C. Wasicki ${ }^{42}$, I. Watanabe ${ }^{66}$, P.M. Watkins ${ }^{18}$, A.T. Watson ${ }^{18}$, I.J. Watson ${ }^{150}$, M.F. Watson ${ }^{18}$,

G. Watts ${ }^{138}$, S. Watts ${ }^{82}$, A.T. Waugh ${ }^{150}$, B.M. Waugh ${ }^{77}$, M.S. Weber ${ }^{17}$, J.S. Webster ${ }^{31}$,

A.R. Weidberg ${ }^{118}$, P. Weigell ${ }^{99}$, J. Weingarten ${ }^{54}$, C. Weiser ${ }^{48}$, P.S. Wells ${ }^{30}$, T. Wenaus ${ }^{25}$,

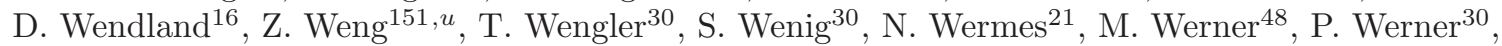

M. Werth ${ }^{163}$, M. Wessels ${ }^{58 a}$, J. Wetter ${ }^{161}$, C. Weydert ${ }^{55}$, K. Whalen ${ }^{29}$, A. White ${ }^{8}$, M.J. White ${ }^{86}$,

S. White ${ }^{122 \mathrm{a}, 122 \mathrm{~b}}$, S.R. Whitehead ${ }^{118}$, D. Whiteson ${ }^{163}$, D. Whittington ${ }^{60}$, F. Wicek ${ }^{115}$,

D. Wicke ${ }^{175}$, F.J. Wickens ${ }^{129}$, W. Wiedenmann ${ }^{173}$, M. Wielers ${ }^{129}$, P. Wienemann ${ }^{21}$,

C. Wiglesworth ${ }^{75}$, L.A.M. Wiik-Fuchs ${ }^{21}$, P.A. Wijeratne ${ }^{77}$, A. Wildauer ${ }^{99}$, M.A. Wildt ${ }^{42, r}$,

I. Wilhelm ${ }^{126}$, H.G. Wilkens ${ }^{30}$, J.Z. Will ${ }^{98}$, E. Williams ${ }^{35}$, H.H. Williams ${ }^{120}$, W. Willis ${ }^{35}$,

S. Willocq ${ }^{84}$, J.A. Wilson ${ }^{18}$, M.G. Wilson ${ }^{143}$, A. Wilson ${ }^{87}$, I. Wingerter-Seez ${ }^{5}$, S. Winkelmann ${ }^{48}$, F. Winklmeier ${ }^{30}$, M. Wittgen ${ }^{143}$, S.J. Wollstadt ${ }^{81}$, M.W. Wolter ${ }^{39}$, H. Wolters ${ }^{124 a}, h$,

W.C. Wong ${ }^{41}$, G. Wooden ${ }^{87}$, B.K. Wosiek ${ }^{39}$, J. Wotschack ${ }^{30}$, M.J. Woudstra ${ }^{82}$, K.W. Wozniak ${ }^{39}$, K. Wraight ${ }^{53}$, M. Wright ${ }^{53}$, B. Wrona ${ }^{73}$, S.L. Wu ${ }^{173}$, X. $\mathrm{Wu}^{49}$, Y. Wu ${ }^{33 b, a k}$, E. Wulf ${ }^{35}$, B.M. Wynne ${ }^{46}$, S. Xella ${ }^{36}$, M. Xiao ${ }^{136}$, S. Xie ${ }^{48}$, C. Xu ${ }^{33 b, y}$, D. Xu ${ }^{139}$, L. Xu $^{33 b}$, B. Yabsley ${ }^{150}$, S. Yacoob ${ }^{145 a, a l}$, M. Yamada ${ }^{65}$, H. Yamaguchi ${ }^{155}$, A. Yamamoto ${ }^{65}$, K. Yamamoto ${ }^{63}$,

S. Yamamoto ${ }^{155}$, T. Yamamura ${ }^{155}$, T. Yamanaka ${ }^{155}$, T. Yamazaki ${ }^{155}$, Y. Yamazaki ${ }^{66}$, Z. Yan $^{22}$, H. Yang ${ }^{87}$, U.K. Yang ${ }^{82}$, Y. Yang ${ }^{109}$, Z. Yang ${ }^{146 a, 146 b}$, S. Yanush ${ }^{91}$, L. Yao ${ }^{33 a}$, Y. Yao ${ }^{15}$,

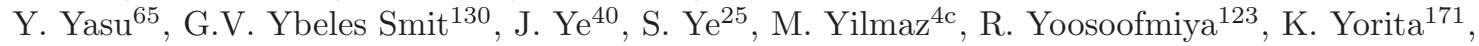

R. Yoshida ${ }^{6}$, K. Yoshihara ${ }^{155}$, C. Young ${ }^{143}$, C.J. Young ${ }^{118}$, S. Youssef ${ }^{22}$, D. Yu ${ }^{25}$, J. Yu $^{8}$,

J. Yu ${ }^{112}$, L. Yuan $^{66}$, A. Yurkewicz ${ }^{106}$, B. Zabinski ${ }^{39}$, R. Zaidan ${ }^{62}$, A.M. Zaitsev ${ }^{128}$, Z. Zajacova ${ }^{30}$, L. Zanello ${ }^{132 a, 132 b}$, D. Zanzi ${ }^{99}$, A. Zaytsev ${ }^{25}$, C. Zeitnitz ${ }^{175}$, M. Zeman ${ }^{125}$, A. Zemla ${ }^{39}$,

C. Zendler ${ }^{21}$, O. Zenin ${ }^{128}$, T. Ženišš ${ }^{144 a}$, Z. Zinonos ${ }^{122 a, 122 b}$, D. Zerwas ${ }^{115}$, G. Zevi della Porta ${ }^{57}$,

D. Zhang ${ }^{33 b, a j}$, H. Zhang ${ }^{88}$, J. Zhang ${ }^{6}$, X. Zhang ${ }^{33 d}$, Z. Zhang ${ }^{115}$, L. Zhao ${ }^{108}$, Z. Zhao ${ }^{33 b}$,

A. Zhemchugov ${ }^{64}$, J. Zhong ${ }^{118}$, B. Zhou ${ }^{87}$, N. Zhou ${ }^{163}$, Y. Zhou ${ }^{151}$, C.G. Zhu ${ }^{33 d}$, H. Zhu ${ }^{42}$,

J. Zhu ${ }^{87}$, Y. Zhu ${ }^{33 b}$, X. Zhuang ${ }^{98}$, V. Zhuravlov ${ }^{99}$, A. Zibell ${ }^{98}$, D. Zieminska ${ }^{60}$, N.I. Zimin ${ }^{64}$,

R. Zimmermann ${ }^{21}$, S. Zimmermann ${ }^{21}$, S. Zimmermann ${ }^{48}$, M. Ziolkowski ${ }^{141}$, R. Zitoun ${ }^{5}$,

L. Živković ${ }^{35}$, V.V. Zmouchko ${ }^{128, *}$, G. Zobernig ${ }^{173}$, A. Zoccoli ${ }^{20 a, 20 b}$, M. zur Nedden ${ }^{16}$,

V. Zutshi ${ }^{106}$, L. Zwalinski ${ }^{30}$

1 School of Chemistry and Physics, University of Adelaide, Adelaide, Australia

2 Physics Department, SUNY Albany, Albany NY, United States of America

3 Department of Physics, University of Alberta, Edmonton AB, Canada

4 (a) Department of Physics, Ankara University, Ankara; ${ }^{(b)}$ Department of Physics, Dumlupinar University, Kutahya; ${ }^{(c)}$ Department of Physics, Gazi University, Ankara; ${ }^{(d)}$ Division of Physics, TOBB University of Economics and Technology, Ankara; ${ }^{(e)}$ Turkish Atomic Energy Authority, Ankara, Turkey

5 LAPP, CNRS/IN2P3 and Université de Savoie, Annecy-le-Vieux, France

6 High Energy Physics Division, Argonne National Laboratory, Argonne IL, United States of America

7 Department of Physics, University of Arizona, Tucson AZ, United States of America

8 Department of Physics, The University of Texas at Arlington, Arlington TX, United States of America

9 Physics Department, University of Athens, Athens, Greece

10 Physics Department, National Technical University of Athens, Zografou, Greece

11 Institute of Physics, Azerbaijan Academy of Sciences, Baku, Azerbaijan 
12 Institut de Física d'Altes Energies and Departament de Física de la Universitat Autònoma de Barcelona and ICREA, Barcelona, Spain

13 (a) Institute of Physics, University of Belgrade, Belgrade; ${ }^{(b)}$ Vinca Institute of Nuclear Sciences, University of Belgrade, Belgrade, Serbia

14 Department for Physics and Technology, University of Bergen, Bergen, Norway

15 Physics Division, Lawrence Berkeley National Laboratory and University of California, Berkeley CA, United States of America

16 Department of Physics, Humboldt University, Berlin, Germany

17 Albert Einstein Center for Fundamental Physics and Laboratory for High Energy Physics, University of Bern, Bern, Switzerland

18 School of Physics and Astronomy, University of Birmingham, Birmingham, United Kingdom

19 (a) Department of Physics, Bogazici University, Istanbul; ${ }^{(b)}$ Division of Physics, Dogus University, Istanbul; ${ }^{(c)}$ Department of Physics Engineering, Gaziantep University, Gaziantep; ${ }^{(d)}$ Department of Physics, Istanbul Technical University, Istanbul, Turkey

20 (a) INFN Sezione di Bologna; ${ }^{(b)}$ Dipartimento di Fisica, Università di Bologna, Bologna, Italy

21 Physikalisches Institut, University of Bonn, Bonn, Germany

22 Department of Physics, Boston University, Boston MA, United States of America

23 Department of Physics, Brandeis University, Waltham MA, United States of America

24 (a) Universidade Federal do Rio De Janeiro COPPE/EE/IF, Rio de Janeiro; ${ }^{(b)}$ Federal University of Juiz de Fora (UFJF), Juiz de Fora; ${ }^{(c)}$ Federal University of Sao Joao del Rei (UFSJ), Sao Joao del Rei; ${ }^{(d)}$ Instituto de Fisica, Universidade de Sao Paulo, Sao Paulo, Brazil

25 Physics Department, Brookhaven National Laboratory, Upton NY, United States of America

26 (a) National Institute of Physics and Nuclear Engineering, Bucharest; ${ }^{(b)}$ University Politehnica Bucharest, Bucharest; ${ }^{(c)}$ West University in Timisoara, Timisoara, Romania

27 Departamento de Física, Universidad de Buenos Aires, Buenos Aires, Argentina

28 Cavendish Laboratory, University of Cambridge, Cambridge, United Kingdom

29 Department of Physics, Carleton University, Ottawa ON, Canada

30 CERN, Geneva, Switzerland

31 Enrico Fermi Institute, University of Chicago, Chicago IL, United States of America

32 (a) Departamento de Física, Pontificia Universidad Católica de Chile, Santiago; ${ }^{(b)}$ Departamento de Física, Universidad Técnica Federico Santa María, Valparaíso, Chile

33 (a) Institute of High Energy Physics, Chinese Academy of Sciences, Beijing; ${ }^{(b)}$ Department of Modern Physics, University of Science and Technology of China, Anhui; ${ }^{(c)}$ Department of Physics, Nanjing University, Jiangsu; ${ }^{(d)}$ School of Physics, Shandong University, Shandong, China

34 Laboratoire de Physique Corpusculaire, Clermont Université and Université Blaise Pascal and CNRS/IN2P3, Clermont-Ferrand, France

35 Nevis Laboratory, Columbia University, Irvington NY, United States of America

36 Niels Bohr Institute, University of Copenhagen, Kobenhavn, Denmark

37 (a) INFN Gruppo Collegato di Cosenza; ${ }^{(b)}$ Dipartimento di Fisica, Università della Calabria, Arcavata di Rende, Italy

38 AGH University of Science and Technology, Faculty of Physics and Applied Computer Science, Krakow, Poland

39 The Henryk Niewodniczanski Institute of Nuclear Physics, Polish Academy of Sciences, Krakow, Poland

40 Physics Department, Southern Methodist University, Dallas TX, United States of America

41 Physics Department, University of Texas at Dallas, Richardson TX, United States of America

42 DESY, Hamburg and Zeuthen, Germany

43 Institut für Experimentelle Physik IV, Technische Universität Dortmund, Dortmund, Germany

44 Institut für Kern- und Teilchenphysik, Technical University Dresden, Dresden, Germany

45 Department of Physics, Duke University, Durham NC, United States of America

46 SUPA - School of Physics and Astronomy, University of Edinburgh, Edinburgh, United Kingdom

47 INFN Laboratori Nazionali di Frascati, Frascati, Italy 
48 Fakultät für Mathematik und Physik, Albert-Ludwigs-Universität, Freiburg, Germany

49 Section de Physique, Université de Genève, Geneva, Switzerland

50 (a) INFN Sezione di Genova; ${ }^{(b)}$ Dipartimento di Fisica, Università di Genova, Genova, Italy

$51{ }^{(a)}$ E. Andronikashvili Institute of Physics, Iv. Javakhishvili Tbilisi State University, Tbilisi; ${ }^{(b)}$ High Energy Physics Institute, Tbilisi State University, Tbilisi, Georgia

52 II Physikalisches Institut, Justus-Liebig-Universität Giessen, Giessen, Germany

53 SUPA - School of Physics and Astronomy, University of Glasgow, Glasgow, United Kingdom

54 II Physikalisches Institut, Georg-August-Universität, Göttingen, Germany

55 Laboratoire de Physique Subatomique et de Cosmologie, Université Joseph Fourier and CNRS/IN2P3 and Institut National Polytechnique de Grenoble, Grenoble, France

56 Department of Physics, Hampton University, Hampton VA, United States of America

57 Laboratory for Particle Physics and Cosmology, Harvard University, Cambridge MA, United States of America

58 (a) Kirchhoff-Institut für Physik, Ruprecht-Karls-Universität Heidelberg, Heidelberg; ${ }^{(b)}$ Physikalisches Institut, Ruprecht-Karls-Universität Heidelberg, Heidelberg; ${ }^{(c)}$ ZITI Institut für technische Informatik, Ruprecht-Karls-Universität Heidelberg, Mannheim, Germany

59 Faculty of Applied Information Science, Hiroshima Institute of Technology, Hiroshima, Japan

60 Department of Physics, Indiana University, Bloomington IN, United States of America

61 Institut für Astro- und Teilchenphysik, Leopold-Franzens-Universität, Innsbruck, Austria

62 University of Iowa, Iowa City IA, United States of America

63 Department of Physics and Astronomy, Iowa State University, Ames IA, United States of America

64 Joint Institute for Nuclear Research, JINR Dubna, Dubna, Russia

65 KEK, High Energy Accelerator Research Organization, Tsukuba, Japan

66 Graduate School of Science, Kobe University, Kobe, Japan

67 Faculty of Science, Kyoto University, Kyoto, Japan

68 Kyoto University of Education, Kyoto, Japan

69 Department of Physics, Kyushu University, Fukuoka, Japan

70 Instituto de Física La Plata, Universidad Nacional de La Plata and CONICET, La Plata, Argentina

71 Physics Department, Lancaster University, Lancaster, United Kingdom

72 (a) INFN Sezione di Lecce; ${ }^{(b)}$ Dipartimento di Matematica e Fisica, Università del Salento, Lecce, Italy

73 Oliver Lodge Laboratory, University of Liverpool, Liverpool, United Kingdom

74 Department of Physics, Jožef Stefan Institute and University of Ljubljana, Ljubljana, Slovenia

75 School of Physics and Astronomy, Queen Mary University of London, London, United Kingdom

76 Department of Physics, Royal Holloway University of London, Surrey, United Kingdom

77 Department of Physics and Astronomy, University College London, London, United Kingdom

78 Laboratoire de Physique Nucléaire et de Hautes Energies, UPMC and Université Paris-Diderot and CNRS/IN2P3, Paris, France

79 Fysiska institutionen, Lunds universitet, Lund, Sweden

80 Departamento de Fisica Teorica C-15, Universidad Autonoma de Madrid, Madrid, Spain

81 Institut für Physik, Universität Mainz, Mainz, Germany

82 School of Physics and Astronomy, University of Manchester, Manchester, United Kingdom

83 CPPM, Aix-Marseille Université and CNRS/IN2P3, Marseille, France

84 Department of Physics, University of Massachusetts, Amherst MA, United States of America

85 Department of Physics, McGill University, Montreal QC, Canada

86 School of Physics, University of Melbourne, Victoria, Australia

87 Department of Physics, The University of Michigan, Ann Arbor MI, United States of America

88 Department of Physics and Astronomy, Michigan State University, East Lansing MI, United States of America

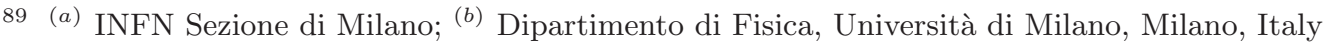

90 B.I. Stepanov Institute of Physics, National Academy of Sciences of Belarus, Minsk, Republic of Belarus 
91 National Scientific and Educational Centre for Particle and High Energy Physics, Minsk, Republic of Belarus

92 Department of Physics, Massachusetts Institute of Technology, Cambridge MA, United States of America

93 Group of Particle Physics, University of Montreal, Montreal QC, Canada

94 P.N. Lebedev Institute of Physics, Academy of Sciences, Moscow, Russia

95 Institute for Theoretical and Experimental Physics (ITEP), Moscow, Russia

96 Moscow Engineering and Physics Institute (MEPhI), Moscow, Russia

97 Skobeltsyn Institute of Nuclear Physics, Lomonosov Moscow State University, Moscow, Russia

98 Fakultät für Physik, Ludwig-Maximilians-Universität München, München, Germany

99 Max-Planck-Institut für Physik (Werner-Heisenberg-Institut), München, Germany

100 Nagasaki Institute of Applied Science, Nagasaki, Japan

101 Graduate School of Science and Kobayashi-Maskawa Institute, Nagoya University, Nagoya, Japan

102 (a) INFN Sezione di Napoli; ${ }^{(b)}$ Dipartimento di Scienze Fisiche, Università di Napoli, Napoli, Italy

103 Department of Physics and Astronomy, University of New Mexico, Albuquerque NM, United States of America

104 Institute for Mathematics, Astrophysics and Particle Physics, Radboud University Nijmegen/Nikhef, Nijmegen, Netherlands

105 Nikhef National Institute for Subatomic Physics and University of Amsterdam, Amsterdam, Netherlands

106 Department of Physics, Northern Illinois University, DeKalb IL, United States of America

107 Budker Institute of Nuclear Physics, SB RAS, Novosibirsk, Russia

108 Department of Physics, New York University, New York NY, United States of America

109 Ohio State University, Columbus OH, United States of America

110 Faculty of Science, Okayama University, Okayama, Japan

111 Homer L. Dodge Department of Physics and Astronomy, University of Oklahoma, Norman OK, United States of America

112 Department of Physics, Oklahoma State University, Stillwater OK, United States of America

113 Palacký University, RCPTM, Olomouc, Czech Republic

114 Center for High Energy Physics, University of Oregon, Eugene OR, United States of America

115 LAL, Université Paris-Sud and CNRS/IN2P3, Orsay, France

116 Graduate School of Science, Osaka University, Osaka, Japan

117 Department of Physics, University of Oslo, Oslo, Norway

118 Department of Physics, Oxford University, Oxford, United Kingdom

119 (a) INFN Sezione di Pavia; ${ }^{(b)}$ Dipartimento di Fisica, Università di Pavia, Pavia, Italy

120 Department of Physics, University of Pennsylvania, Philadelphia PA, United States of America

121 Petersburg Nuclear Physics Institute, Gatchina, Russia

122 (a) INFN Sezione di Pisa; ${ }^{(b)}$ Dipartimento di Fisica E. Fermi, Università di Pisa, Pisa, Italy

123 Department of Physics and Astronomy, University of Pittsburgh, Pittsburgh PA, United States of America

$124{ }^{(a)}$ Laboratorio de Instrumentacao e Fisica Experimental de Particulas - LIP, Lisboa, Portugal; ${ }^{(b)}$ Departamento de Fisica Teorica y del Cosmos and CAFPE, Universidad de Granada, Granada, Spain

125 Institute of Physics, Academy of Sciences of the Czech Republic, Praha, Czech Republic

126 Faculty of Mathematics and Physics, Charles University in Prague, Praha, Czech Republic

127 Czech Technical University in Prague, Praha, Czech Republic

128 State Research Center Institute for High Energy Physics, Protvino, Russia

129 Particle Physics Department, Rutherford Appleton Laboratory, Didcot, United Kingdom

130 Physics Department, University of Regina, Regina SK, Canada

131 Ritsumeikan University, Kusatsu, Shiga, Japan

$132{ }^{(a)}$ INFN Sezione di Roma I; ${ }^{(b)}$ Dipartimento di Fisica, Università La Sapienza, Roma, Italy

133 (a) INFN Sezione di Roma Tor Vergata; ${ }^{(b)}$ Dipartimento di Fisica, Università di Roma Tor Vergata, Roma, Italy 
134 (a) INFN Sezione di Roma Tre; ${ }^{(b)}$ Dipartimento di Fisica, Università Roma Tre, Roma, Italy

135 (a) Faculté des Sciences Ain Chock, Réseau Universitaire de Physique des Hautes Energies -

Université Hassan II, Casablanca; ${ }^{(b)}$ Centre National de l'Energie des Sciences Techniques

Nucleaires, Rabat; ${ }^{(c)}$ Faculté des Sciences Semlalia, Université Cadi Ayyad, LPHEA-Marrakech;

(d) Faculté des Sciences, Université Mohamed Premier and LPTPM, Oujda; ${ }^{(e)}$ Faculté des sciences, Université Mohammed V-Agdal, Rabat, Morocco

136 DSM/IRFU (Institut de Recherches sur les Lois Fondamentales de l'Univers), CEA Saclay

(Commissariat a l'Energie Atomique), Gif-sur-Yvette, France

137 Santa Cruz Institute for Particle Physics, University of California Santa Cruz, Santa Cruz CA, United States of America

138 Department of Physics, University of Washington, Seattle WA, United States of America

139 Department of Physics and Astronomy, University of Sheffield, Sheffield, United Kingdom

140 Department of Physics, Shinshu University, Nagano, Japan

141 Fachbereich Physik, Universität Siegen, Siegen, Germany

142 Department of Physics, Simon Fraser University, Burnaby BC, Canada

143 SLAC National Accelerator Laboratory, Stanford CA, United States of America

144 (a) Faculty of Mathematics, Physics \& Informatics, Comenius University, Bratislava; ${ }^{(b)}$

Department of Subnuclear Physics, Institute of Experimental Physics of the Slovak Academy of Sciences, Kosice, Slovak Republic

145 (a) Department of Physics, University of Johannesburg, Johannesburg; ${ }^{(b)}$ School of Physics, University of the Witwatersrand, Johannesburg, South Africa

146 (a) Department of Physics, Stockholm University; ${ }^{(b)}$ The Oskar Klein Centre, Stockholm, Sweden

147 Physics Department, Royal Institute of Technology, Stockholm, Sweden

148 Departments of Physics \& Astronomy and Chemistry, Stony Brook University, Stony Brook NY, United States of America

149 Department of Physics and Astronomy, University of Sussex, Brighton, United Kingdom

150 School of Physics, University of Sydney, Sydney, Australia

151 Institute of Physics, Academia Sinica, Taipei, Taiwan

152 Department of Physics, Technion: Israel Institute of Technology, Haifa, Israel

153 Raymond and Beverly Sackler School of Physics and Astronomy, Tel Aviv University, Tel Aviv, Israel

154 Department of Physics, Aristotle University of Thessaloniki, Thessaloniki, Greece

155 International Center for Elementary Particle Physics and Department of Physics, The University of Tokyo, Tokyo, Japan

156 Graduate School of Science and Technology, Tokyo Metropolitan University, Tokyo, Japan

157 Department of Physics, Tokyo Institute of Technology, Tokyo, Japan

158 Department of Physics, University of Toronto, Toronto ON, Canada

159 (a) TRIUMF, Vancouver BC; ${ }^{(b)}$ Department of Physics and Astronomy, York University, Toronto ON, Canada

160 Faculty of Pure and Applied Sciences, University of Tsukuba, Tsukuba, Japan

161 Department of Physics and Astronomy, Tufts University, Medford MA, United States of America

162 Centro de Investigaciones, Universidad Antonio Narino, Bogota, Colombia

163 Department of Physics and Astronomy, University of California Irvine, Irvine CA, United States of America

164 (a) INFN Gruppo Collegato di Udine; ${ }^{(b)}$ ICTP, Trieste; ${ }^{(c)}$ Dipartimento di Chimica, Fisica e Ambiente, Università di Udine, Udine, Italy

165 Department of Physics, University of Illinois, Urbana IL, United States of America

166 Department of Physics and Astronomy, University of Uppsala, Uppsala, Sweden

167 Instituto de Física Corpuscular (IFIC) and Departamento de Física Atómica, Molecular y Nuclear and Departamento de Ingeniería Electrónica and Instituto de Microelectrónica de Barcelona (IMB-CNM), University of Valencia and CSIC, Valencia, Spain

168 Department of Physics, University of British Columbia, Vancouver BC, Canada 
169 Department of Physics and Astronomy, University of Victoria, Victoria BC, Canada

170 Department of Physics, University of Warwick, Coventry, United Kingdom

171 Waseda University, Tokyo, Japan

172 Department of Particle Physics, The Weizmann Institute of Science, Rehovot, Israel

173 Department of Physics, University of Wisconsin, Madison WI, United States of America

174 Fakultät für Physik und Astronomie, Julius-Maximilians-Universität, Würzburg, Germany

175 Fachbereich C Physik, Bergische Universität Wuppertal, Wuppertal, Germany

176 Department of Physics, Yale University, New Haven CT, United States of America

177 Yerevan Physics Institute, Yerevan, Armenia

178 Centre de Calcul de l'Institut National de Physique Nucléaire et de Physique des Particules (IN2P3), Villeurbanne, France

a Also at Laboratorio de Instrumentacao e Fisica Experimental de Particulas - LIP, Lisboa, Portugal

${ }^{b}$ Also at Faculdade de Ciencias and CFNUL, Universidade de Lisboa, Lisboa, Portugal

Also at Particle Physics Department, Rutherford Appleton Laboratory, Didcot, United Kingdom

$d$ Also at TRIUMF, Vancouver BC, Canada

$e$ Also at Department of Physics, California State University, Fresno CA, United States of America

$f$ Also at Novosibirsk State University, Novosibirsk, Russia

$g$ Also at Fermilab, Batavia IL, United States of America

$h$ Also at Department of Physics, University of Coimbra, Coimbra, Portugal

Also at Department of Physics, UASLP, San Luis Potosi, Mexico

Also at Università di Napoli Parthenope, Napoli, Italy

Also at Institute of Particle Physics (IPP), Canada

${ }^{l}$ Also at Department of Physics, Middle East Technical University, Ankara, Turkey

$m$ Also at Louisiana Tech University, Ruston LA, United States of America

$n$ Also at Dep Fisica and CEFITEC of Faculdade de Ciencias e Tecnologia, Universidade Nova de Lisboa, Caparica, Portugal

- Also at Department of Physics and Astronomy, University College London, London, United Kingdom

$p$ Also at Department of Physics, University of Cape Town, Cape Town, South Africa

$q$ Also at Institute of Physics, Azerbaijan Academy of Sciences, Baku, Azerbaijan

$r$ Also at Institut für Experimentalphysik, Universität Hamburg, Hamburg, Germany

Also at Manhattan College, New York NY, United States of America

${ }^{t}$ Also at CPPM, Aix-Marseille Université and CNRS/IN2P3, Marseille, France

${ }^{u}$ Also at School of Physics and Engineering, Sun Yat-sen University, Guanzhou, China

$v$ Also at Academia Sinica Grid Computing, Institute of Physics, Academia Sinica, Taipei, Taiwan

$w$ Also at School of Physics, Shandong University, Shandong, China

$x$ Also at Dipartimento di Fisica, Università La Sapienza, Roma, Italy

y Also at DSM/IRFU (Institut de Recherches sur les Lois Fondamentales de l'Univers), CEA Saclay (Commissariat a l'Energie Atomique), Gif-sur-Yvette, France

$z$ Also at Section de Physique, Université de Genève, Geneva, Switzerland

aa Also at Departamento de Fisica, Universidade de Minho, Braga, Portugal

$a b$ Also at Department of Physics and Astronomy, University of South Carolina, Columbia SC, United States of America

ac Also at Institute for Particle and Nuclear Physics, Wigner Research Centre for Physics, Budapest, Hungary

ad Also at California Institute of Technology, Pasadena CA, United States of America

ae Also at Institute of Physics, Jagiellonian University, Krakow, Poland

af Also at LAL, Université Paris-Sud and CNRS/IN2P3, Orsay, France

ag Also at Nevis Laboratory, Columbia University, Irvington NY, United States of America

ah Also at Department of Physics and Astronomy, University of Sheffield, Sheffield, United Kingdom

ai Also at Department of Physics, Oxford University, Oxford, United Kingdom 
aj Also at Institute of Physics, Academia Sinica, Taipei, Taiwan

${ }^{a k}$ Also at Department of Physics, The University of Michigan, Ann Arbor MI, United States of America

al Also at Discipline of Physics, University of KwaZulu-Natal, Durban, South Africa

* Deceased 